\title{
Measurement of charm and beauty production at central rapidity versus charged-particle multiplicity in proton-proton collisions at $\sqrt{\mathrm{s}}=7 \mathrm{TeV}$
}

ALICE

\section{The ALICE collaboration}

\section{E-mail: ALICE-publications@cern.ch}

Abstract: Prompt D meson and non-prompt $\mathrm{J} / \psi$ yields are studied as a function of the multiplicity of charged particles produced in inelastic proton-proton collisions at a centreof-mass energy of $\sqrt{s}=7 \mathrm{TeV}$. The results are reported as a ratio between yields in a given multiplicity interval normalised to the multiplicity-integrated ones (relative yields). They are shown as a function of the multiplicity of charged particles normalised to the average value for inelastic collisions (relative charged-particle multiplicity). $\mathrm{D}^{0}, \mathrm{D}^{+}$and $\mathrm{D}^{*+}$ mesons are measured in five $p_{\mathrm{T}}$ intervals from $1 \mathrm{GeV} / c$ to $20 \mathrm{GeV} / c$ and for $|y|<0.5$ via their hadronic decays. The D-meson relative yield is found to increase with increasing charged-particle multiplicity. For events with multiplicity six times higher than the average multiplicity of inelastic collisions, a yield enhancement of a factor about 15 relative to the multiplicity-integrated yield in inelastic collisions is observed. The yield enhancement is independent of transverse momentum within the uncertainties of the measurement. The $\mathrm{D}^{0}$-meson relative yield is also measured as a function of the relative multiplicity at forward pseudo-rapidity. The non-prompt $\mathrm{J} / \psi$, i.e. the $\mathrm{B}$ hadron, contribution to the inclusive $\mathrm{J} / \psi$ production is measured in the di-electron decay channel at central rapidity. It is evaluated for $p_{\mathrm{T}}>1.3 \mathrm{GeV} / c$ and $|y|<0.9$, and extrapolated to $p_{\mathrm{T}}>0$. The fraction of non-prompt $\mathrm{J} / \psi$ in the inclusive $\mathrm{J} / \psi$ yields shows no dependence on the charged-particle multiplicity at central rapidity. Charm and beauty hadron relative yields exhibit a similar increase with increasing charged-particle multiplicity. The measurements are compared to PYTHIA 8, EPOS 3 and percolation calculations.

KEYwORDS: Hadron-Hadron Scattering

ARXIV EPRINT: 1505.00664 


\section{Contents}

1 Introduction 1

2 Experimental apparatus and data sample $\quad 4$

$\begin{array}{lll}3 & \text { Multiplicity definition and corrections } & 6\end{array}$

4 D-meson analysis $\quad 7$

$\begin{array}{lll}4.1 & \text { D-meson reconstruction } & 7\end{array}$

$\begin{array}{llr}4.2 & \text { Corrections } & 9\end{array}$

$\begin{array}{lll}4.3 & \text { Systematic uncertainties } & 11\end{array}$

4.4 Results 12

4.4.1 Studies with the charged-particle multiplicity at forward rapidity 13

5 Non-prompt $\mathrm{J} / \psi$ analysis $\quad 16$

5.1 Non-prompt $\mathrm{J} / \psi$ reconstruction 16

$\begin{array}{lll}5.2 \text { Corrections } & 18\end{array}$

$\begin{array}{lll}5.3 & \text { Systematic uncertainties } & 19\end{array}$

$\begin{array}{lll}5.4 \text { Results } & 21\end{array}$

6 Comparison of charm and beauty production $\quad 21$

7 Comparison to theoretical calculations $\quad 24$

7.1 PYTHIA 8 simulations 24

$\begin{array}{ll}7.2 & \text { Comparison of data with models } \\ \end{array}$

$\begin{array}{llr}8 & \text { Summary } & 29\end{array}$

$\begin{array}{ll}\text { A Tables of the results } & 31\end{array}$

$\begin{array}{lr}\text { The ALICE collaboration } & 39\end{array}$

\section{Introduction}

The study of the production of hadrons containing heavy quarks, i.e. charm and beauty, in proton-proton (pp) collisions at the Large Hadron Collider (LHC) provides a way to test calculations based on perturbative Quantum Chromodynamics (pQCD) at the highest available collision energies. The inclusive production cross sections of charm mesons measured in pp collisions at the LHC at both central [1,2] and forward [3] rapidity are described by theoretical predictions based on pQCD calculations with the collinear factorisation approach at next-to-leading order (e.g. in the general-mass variable-flavour-number 
scheme, GM-VFNS [4]) or at fixed order with next-to-leading-log resummation (FONLL [58]) within theoretical uncertainties. The comparisons suggest that charm production is under (over) estimated by the central values of the FONLL (GM-VFNS) calculations. The measured D-meson production cross sections in pp collisions at the LHC can also be described by pQCD calculations performed in the framework of $k_{\mathrm{T}}$-factorisation in the leading order (LO) approximation [9]. Beauty production cross section measurements in pp collisions at $\sqrt{s}=7 \mathrm{TeV}$ [10-14] are well described by implementations of FONLL and GM-VFNS [7, 15]. In the case of B mesons, the measured cross sections are close to the central value of the FONLL and GM-VFNS predictions. A similar situation was observed in $\mathrm{p} \overline{\mathrm{p}}$ collisions at $\sqrt{s}=1.96 \mathrm{TeV}$ at the FNAL Tevatron collider [16-18].

The measurement of heavy-flavour production in pp collisions as a function of the charged-particle multiplicity produced in the collision could provide insight into the processes occurring in the collision at the partonic level and the interplay between the hard and soft mechanisms in particle production. These aspects are expected to depend on the energy and on the impact parameter (the distance between the colliding protons in the plane perpendicular to the beam direction) of the pp collision [19-21]. In the impact parameter representation of proton-proton collisions, the overlap of the nucleon wave functions in proton-proton collisions can be described by a geometrical picture with two separate transverse distance scales: the impact parameter of the collision and the transverse spatial partonic distribution [20, 22-24]. In particular, pp collisions with a hard partonparton scattering are predicted to be more central (i.e. have smaller impact parameter) than minimum-bias events $[20,25]$.

The NA27 Collaboration observed in 1988 that the average charged-particle multiplicity in events with open charm production was higher by about $20 \%$ than in events without charm production [26]. A softening of the momentum spectra of hadrons produced in association with charm was also observed. This result was interpreted as a consequence of the more central nature of collisions leading to charm production.

At LHC energies, two additional contributions to charm production and its relation to multiplicity have to be considered. The first effect is the likely larger amount of gluon radiation associated to the short distance production processes at larger energies and particle transverse momenta. The second is the contribution of Multiple-Parton Interactions (MPI) [27-29], i.e. several hard partonic interactions occurring in a single pp collision. In this context, pQCD-inspired models describe the final-state particles produced in hadronic collisions with a two-component approach, namely an initial hard partonic scattering process, that gives rise to collimated clusters of hadrons (jets), and an underlying event, consisting of the final-state particles that are not associated with the initial hard scattering. While the hard scattering process can be computed with a pQCD approach, the description of the underlying event, which is thought to be dominated by particles produced in soft processes and by perturbative (mini)jets with relatively small transverse momenta (soft MPIs), is based on a phenomenological model. In particular, pQCD-based models of MPIs provide a consistent way to describe high multiplicity pp collisions, and have been implemented in recent Monte Carlo generators like PYTHIA 6 [30], PYTHIA 8 [31], and HERWIG [32]. Measurements by the CMS Collaboration of jet and underlying event prop- 
erties as a function of multiplicity in pp collisions at $\sqrt{s}=7 \mathrm{TeV}$ can be better described by event generators including MPI $[33,34]$. The analysis of minijet production performed by the ALICE Collaboration [35] indicates that high multiplicities in pp collisions are reached through a high number of MPIs and a higher than average number of fragments per parton. Upward fluctuations of the gluon density in the colliding protons are also advocated to describe the results from high multiplicity pp collisions at the LHC [21, 36, 37]. Indeed, the transverse structure of the proton, as probed in hard partonic scattering processes, is predicted to play a crucial role in defining the underlying event structure and the probability of MPIs [25]. In the heavy-flavour sector, the LHCb Collaboration reported measurements of double charm production in pp collisions at the LHC $\left(\mathrm{D}^{0}+X, \mathrm{~J} / \psi+X\right.$ and $\mathrm{J} / \psi+\mathrm{J} / \psi$ where $X=\mathrm{D}^{0}, \mathrm{D}^{+}, \mathrm{D}_{\mathrm{s}}^{+}, \Lambda_{\mathrm{c}}^{+}$), which suggest that MPIs also play a role at the hard momentum scale relevant for $c \bar{c}$ production $[38,39]$.

The ALICE Collaboration published the first measurement of inclusive $\mathrm{J} / \psi$ production as a function of charged-particle multiplicity, expressed as the pseudo-rapidity density of charged particles $\mathrm{d} N_{\mathrm{ch}} / \mathrm{d} \eta$ at mid-rapidity, in pp collisions at $\sqrt{s}=7 \mathrm{TeV}$ [40]. An approximately linear increase of the yield of $\mathrm{J} / \psi$ with the charged-particle multiplicity was observed in a multiplicity range reaching four times the average multiplicity $\left\langle\mathrm{d} N_{\mathrm{ch}} / \mathrm{d} \eta\right\rangle$. The measurements at $|y|<0.9$ and $2.5<y<4.0$ were compatible within the uncertainties. Both the larger amount of gluon radiation and the contribution of MPI in collisions where heavy quarks are produced can induce a correlation between the yield of quarkonia and the charged-particle multiplicity produced in the collision. The measured rise of $\mathrm{J} / \psi$ yield with increasing multiplicity can also be described in the framework of string interaction or parton saturation models. In particular, in ref. [41] a stronger-than-linear trend in the high density domain is anticipated as a consequence of the interaction (overlap) of strings, which reduces the effective number of sources for soft-particle production. The increasing trend of $\mathrm{J} / \psi$ yield with multiplicity is also described in a framework in which high multiplicities are attained in pp collisions due to the contribution of higher Fock states in the proton, leading to a larger number of gluons participating in the collision [37].

It is also worth pointing out that the charged-particle densities attained in highmultiplicity pp collisions at the LHC are of the same order of magnitude as those measured in semi-peripheral heavy-ion collisions at lower centre-of-mass energies [42]. In those heavyion collisions, the measured momentum distributions of light hadrons indicate that the system undergoes a collective expansion, which can be described in terms of hydrodynamics. Recent measurements in high-multiplicity p-Pb collisions at $\sqrt{s_{\mathrm{NN}}}=5.02 \mathrm{TeV}[43-48]$ and in high-multiplicity pp collisions at the LHC [49] indicate that such a collective behaviour could also be at play in these systems. If charm quarks were to follow a collective motion in high-multiplicity events, their momentum spectra would be altered, and the heavy-flavour hadron relative yields at high multiplicity would vary as a function of $p_{\mathrm{T}}$ [50].

The measurements of the $p_{\mathrm{T}}$-differential prompt $\mathrm{D}$ meson and non-prompt $\mathrm{J} / \psi$ cross sections in pp collisions at $\sqrt{s}=7 \mathrm{TeV}$ with the ALICE experiment at the LHC were published in references $[1,10]$. In this paper, we report the measurement of the relative open heavy-flavour production yields as a function of the charged-particle multiplicity in pp collisions at $\sqrt{s}=7 \mathrm{TeV}$. Open charm and beauty production is measured by recon- 
structing prompt $\mathrm{D}$ mesons and non-prompt $\mathrm{J} / \psi$, i.e. $\mathrm{J} / \psi$ mesons coming from the decay of beauty hadrons. The experimental setup and the multiplicity estimation are described in sections 2 and 3 , respectively. Prompt $\mathrm{D}^{0}, \mathrm{D}^{+}, \mathrm{D}^{*+}$ mesons were measured at central rapidity, $|y|<0.5$, in six multiplicity intervals and five $p_{\mathrm{T}}$ intervals from $1 \mathrm{GeV} / c$ to 20 $\mathrm{GeV} / c$ (section 4). The non-prompt fraction of $\mathrm{J} / \psi$ production was measured in the rapidity interval $|y|<0.9$ in five multiplicity intervals and for $p_{\mathrm{T}}>1.3 \mathrm{GeV} / c$ and extrapolated to $p_{\mathrm{T}}>0$ (section 5 ). The relative yields as a function of charged-particle multiplicity are compared in section 6 . Finally, model calculations are discussed and compared with data in section 7 .

\section{Experimental apparatus and data sample}

The ALICE apparatus [51] consists of a central barrel detector covering the pseudo-rapidity interval $|\eta|<0.9$, a forward muon spectrometer covering the pseudo-rapidity interval $-4.0<\eta<-2.5$, and a set of detectors at forward and backward rapidities used for triggering and event characterization. In the following, the subsystems that are relevant for the $\mathrm{D}$ meson and non-prompt $\mathrm{J} / \psi$ analyses are described.

The central barrel detectors are located inside a large solenoidal magnet, which provides a magnetic field of $0.5 \mathrm{~T}$ along the beam direction ( $z$ axis in the ALICE reference frame). Tracking and particle identification are performed using the information provided by the Inner Tracking System (ITS), the Time Projection Chamber (TPC) and the Time Of Flight (TOF) detectors, that have full azimuthal coverage in the pseudo-rapidity interval $|\eta|<0.9$. The detector closest to the beam axis is the ITS, which is composed of six cylindrical layers of silicon detectors, with radial distances from the beam axis ranging from $3.9 \mathrm{~cm}$ to $43.0 \mathrm{~cm}$. The two innermost layers, with average radii of $3.9 \mathrm{~cm}$ and $7.6 \mathrm{~cm}$, are equipped with Silicon Pixel Detectors (SPD). The two SPD layers, covering the pseudo-rapidity ranges of $|\eta|<2.0$ and $|\eta|<1.4$ respectively, have 1200 SPD readout chips. The two intermediate layers are made of Silicon Drift Detectors (SDD), while Silicon Strip Detectors (SSD) equip the two outermost layers. The high spatial resolution of the silicon sensors, together with the low material budget (on average $7.7 \%$ of a radiation length for tracks crossing the ITS perpendicularly to the detector surfaces, i.e. $\eta=0$ ) and the small distance of the innermost layer from the beam vacuum tube, allow for the measurement of the track impact parameter in the transverse plane $\left(d_{0}\right)$, i.e. the distance of closest approach of the track to the primary vertex in the plane transverse to the beam direction, with a resolution better than $75 \mu \mathrm{m}$ for transverse momenta $p_{\mathrm{T}}>1 \mathrm{GeV} / c[52]$. The SPD provides also a measurement of the multiplicity of charged particles produced in the collision based on track segments (tracklets) built by associating pairs of hits in the two SPD layers.

At larger radii $(85<r<247 \mathrm{~cm})$, a $510 \mathrm{~cm}$ long cylindrical TPC [53] provides track reconstruction with up to 159 three-dimensional space points per track, as well as particle identification via the measurement of the specific energy deposit $\mathrm{d} E / \mathrm{d} x$ in the gas. The charged particle identification capability of the TPC is supplemented by the TOF [54], which is equipped with Multi-gap Resistive Plate Chambers (MRPCs) located 
at radial distances between 377 and $399 \mathrm{~cm}$ from the beam axis. The overall TOF resolution including the uncertainty on the time at which the collision took place, and the tracking and momentum resolution was about $160 \mathrm{ps}$ for the data-taking period considered in these analyses.

The V0 detector [55], used for triggering and for estimating the multiplicity of charged particles in the forward rapidity region, consists of two arrays of 32 scintillators each, placed around the beam vacuum tube on either side of the interaction region at $z=-90 \mathrm{~cm}$ and $z=+340 \mathrm{~cm}$. The two arrays cover the pseudo-rapidity intervals $-3.7<\eta<-1.7$ and $2.8<\eta<5.1$, respectively.

The data from proton-proton (pp) collisions at a centre-of-mass energy of $\sqrt{s}=7 \mathrm{TeV}$ used for the analyses were recorded in 2010. The data sample consists of about 314 million minimum-bias $(\mathrm{MB})$ events, corresponding to an integrated luminosity of $\mathcal{L}_{\text {int }} \simeq 5 \mathrm{nb}^{-1}$. Minimum-bias collisions were triggered by requiring at least one hit in either of the V0 counters or in the SPD $(|\eta|<2)$, in coincidence with the arrival time of proton bunches from both directions. This trigger was estimated to be sensitive to about $85 \%$ of the inelastic cross section [56].

To enrich the data sample with high multiplicity events, a High Multiplicity (HM) trigger based on the multiplicity information provided by the outer SPD layer was also used. Each readout chip of the SPD promptly asserts a digital pulse, called FastOR bit, on the presence of at least one firing pixel. A sample of about 6 million events was collected applying a selection on the minimum number of readout chips having asserted this digital pulse. The threshold was configured to select the $\approx 0.7 \%$ of the events with highest number of hits in the outer SPD layer. This HM-trigger sample $\left(\mathcal{L}_{\text {int }} \simeq 14 \mathrm{nb}^{-1}\right)$ provides an increase of statistics by a factor of about 2.8 relative to the MB trigger for events with more than 50 tracklets, corresponding to about six times the average multiplicity.

Only events with interaction vertex reconstructed from tracks with a coordinate $|z|<$ $10 \mathrm{~cm}$ along the beam line were used for the analysis. In the considered data samples, the instantaneous luminosity was limited to $0.6-1.2 \times 10^{29} \mathrm{~cm}^{-2} \mathrm{~s}^{-1}$ by displacing the beams in the transverse plane by 3.8 times the RMS of their transverse profile. In this way, the interaction probability per bunch crossing was kept in the range $0.04-0.08$, with a probability of collision pile-up below $4 \%$ per triggered event. An algorithm to detect multiple interaction vertices based on SPD track segments, or tracklets, was used to further reduce the pile-up contribution. An event is rejected from the analysed data sample if a second interaction vertex is found, which has at least three associated tracklets, and is separated from the first one by more than $0.8 \mathrm{~cm}$ along $z$. This removes about $48 \%$ of the pile-up events. The remaining pile-up contamination has two contributions: events with pile-up of collisions with $\Delta z<0.8 \mathrm{~cm}$ and events in which the piled-up collisions have low-multiplicity (less than three charged particles reconstructed in the SPD). In the case of pile-up of collisions with small separation along $z$, the multiplicity estimation may be biased because some of the tracklets of charged particles from different interactions may be added together. According to simulations, the number of tracklets results to be biased when the piled-up vertices are separated along $z$ by less than $0.6 \mathrm{~cm}$. Combining this result with the shape of the luminous region along the beam direction and the maximum 
pile-up rate of $4 \%$, the overall probability that two piled-up events induce a bias in the determination of multiplicity was found to be lower than $0.3 \%$. The fraction of events with biased number of tracklets increases with increasing multiplicity and it was estimated to be below $2 \%$ at the highest multiplicities considered in this analysis, while the resulting bias on the measured number of tracklets was found to be negligible in all the multiplicity classes.

\section{Multiplicity definition and corrections}

In the present analysis, the experimental estimator of the charged-particle multiplicity is the number of tracklets in the interval $|\eta|<1.0$ ( $\left.N_{\text {tracklets }}\right)$. Tracklets are track segments defined by combining the clusters in the SPD detector with the reconstructed primary vertex position. Tracklets are required to point to the primary interaction vertex within $\pm 1 \mathrm{~cm}$ in the transverse plane and $\pm 3 \mathrm{~cm}$ in the $z$ direction [51, 52]. This multiplicity estimator is the same as was used in previous studies performed for inclusive $\mathrm{J} / \psi$ production [40]. Monte Carlo simulations of the detector response have shown that $N_{\text {tracklets }}$ is proportional to the pseudo-rapidity density of the generated charged primary particles, $\mathrm{d} N_{\mathrm{ch}} / \mathrm{d} \eta$, within $2 \%$. Primary particles are defined as prompt particles produced in the collision and all decay products, except products from weak decays of strange particles. The pseudo-rapidity coverage of the SPD detector changes with the position of the interaction vertex along the beam line, $z_{\mathrm{vtx}}$, and with time due to the variation of the number of inactive channels. The detector response over the analysed data taking period is equalised by means of a databased correction, which is applied on an event-by-event basis depending on $z_{\mathrm{vtx}}$ and time.

The measurements in the $N_{\text {tracklets }} \in[1,49]$ interval are performed using minimumbias triggered data, while those in the $[50,80]$ range exploit the SPD-based HM trigger described above. The HM trigger is fully efficient for events with $N_{\text {tracklets }}>65$. The number of events and the D-meson candidate invariant mass distributions were corrected for the HM trigger inefficiency in the $N_{\text {tracklets }} \in[50,65]$ range by means of a data-driven re-weighting procedure. The $N_{\text {tracklets-dependent event weights were defined from the ratio }}$ of the measured distributions of the number of tracklets in the HM and minimum-bias trigger samples. The effect of this correction on the per-event raw yield was of about $2.5 \%$. The average $\mathrm{d} N_{\mathrm{ch}} / \mathrm{d} \eta$ of events in the highest $N_{\text {tracklets }}$ interval was determined from the minimum-bias sample.

The analysis results are presented as a function of the relative charged-particle multiplicity at central rapidity, $\left(\mathrm{d} N_{\mathrm{ch}} / \mathrm{d} \eta\right)^{j} /\left\langle\mathrm{d} N_{\mathrm{ch}} / \mathrm{d} \eta\right\rangle$, where $\left\langle\mathrm{d} N_{\mathrm{ch}} / \mathrm{d} \eta\right\rangle=6.01 \pm 0.01$ (stat. $)_{-0.12}^{+0.20}$ (syst.) is measured in inelastic pp collisions at $\sqrt{s}=7 \mathrm{TeV}$ with at least one charged particle in $|\eta|<1.0$ [57]. The relative quantities are used to minimise the experimental uncertainties and to facilitate the comparison with other measurements and models. The considered $N_{\text {tracklets }}$ intervals and the corresponding relative charged-particle multiplicity ranges are summarised in table 1 . The highest $N_{\text {tracklets }}$ interval considered in the analysis extends to a multiplicity of about 9 times the $\left\langle\mathrm{d} N_{\mathrm{ch}} / \mathrm{d} \eta\right\rangle$ of inelastic pp collisions and the average multiplicity of events in this $N_{\text {tracklets }}$ interval is about six times the $\left\langle\mathrm{d} N_{\mathrm{ch}} / \mathrm{d} \eta\right\rangle$. The uncertainty on $\left(\mathrm{d} N_{\mathrm{ch}} / \mathrm{d} \eta\right)^{j} /\left\langle\mathrm{d} N_{\mathrm{ch}} / \mathrm{d} \eta\right\rangle$ is $6 \%$; it includes the influence of (i) the deter- 


\begin{tabular}{|ccccc|}
\hline$N_{\text {tracklets }}$ & $\left(\mathrm{d} N_{\text {ch }} / \mathrm{d} \eta\right)^{j}$ & $\left(\mathrm{~d} N_{\mathrm{ch}} / \mathrm{d} \eta\right)^{j} /\left\langle\mathrm{d} N_{\mathrm{ch}} / \mathrm{d} \eta\right\rangle$ & $N_{\text {events }}^{\mathrm{D}^{0}} / 10^{6}$ & $N_{\text {events }}^{\mathrm{J} / \psi} / 10^{6}$ \\
\hline$[1,8]$ & 2.7 & $0.45_{-0.03}^{+0.03}$ & 155.1 & - \\
{$[4,8]$} & 3.8 & $0.63_{-0.04}^{+0.04}$ & - & 89.0 \\
{$[9,13]$} & 7.1 & $1.18_{-0.07}^{+0.07}$ & 46.2 & 50.5 \\
{$[14,19]$} & 10.7 & $1.78_{-0.11}^{+0.10}$ & 32.0 & 35.5 \\
{$[20,30]$} & 15.8 & $2.63_{-0.17}^{+0.15}$ & 24.7 & 28.0 \\
{$[31,49]$} & 24.1 & $4.01_{-0.25}^{+0.23}$ & 7.9 & 9.5 \\
{$[50,80]$} & 36.7 & $6.11_{-0.39}^{+0.35}$ & 1.7 & - \\
\hline
\end{tabular}

Table 1. Summary of the multiplicity intervals used for the analyses. The number of reconstructed tracklets $N_{\text {tracklets }}$, the average charged-particle multiplicity $\left(\mathrm{d} N_{\mathrm{ch}} / \mathrm{d} \eta\right)^{j}$, and the relative chargedparticle multiplicity $\left(\mathrm{d} N_{\mathrm{ch}} / \mathrm{d} \eta\right)^{j} /\left\langle\mathrm{d} N_{\mathrm{ch}} / \mathrm{d} \eta\right\rangle$ are detailed. The number of events analysed in the various multiplicity ranges for both the D-meson and $\mathrm{J} / \psi$ analyses are reported. The number of events for the $N_{\text {tracklets }}$ interval $[50,80]$ are corrected for the high multiplicity trigger efficiency, as explained in section 3 .

mination of the $N_{\text {tracklets }}$ to $\mathrm{d} N_{\mathrm{ch}} / \mathrm{d} \eta$ proportionality factor, $2 \%$, (ii) its possible deviation from linearity, 5\%, (iii) and the uncertainty on the measured $\left\langle\mathrm{d} N_{\mathrm{ch}} / \mathrm{d} \eta\right\rangle$.

The analysis of $\mathrm{D}^{0}$ production is also carried out as a function of the charged-particle multiplicity in the regions $-3.7<\eta<-1.7$ and $2.8<\eta<5.1$, as measured with the charge collected by the V0 scintillator counters, $N_{\mathrm{V} 0}$, reported in units of the minimum-ionizingparticle charge. The motivation for studying the multiplicity dependence of charmed-meson production also with this estimator is that the event multiplicity and the D-meson yields are evaluated in different pseudorapidity ranges, reducing the effects of auto-correlations. In contrast, with the $N_{\text {tracklets }}$ estimator also the D-meson decay products and the charged particles produced in the fragmentation of the same charm quark are included in the multiplicity evaluation. Monte Carlo simulations demonstrate that $N_{\mathrm{V} 0}$ is proportional to the charged-particle multiplicity in that pseudo-rapidity interval. In this paper we report $\mathrm{D}^{0}$ relative yields as a function of the relative uncorrected multiplicity in the V0 detector, $N_{\mathrm{V} 0} /\left\langle N_{\mathrm{V} 0}\right\rangle$ (see section 4.4.1).

\section{D-meson analysis}

\subsection{D-meson reconstruction}

Charm production was studied by reconstructing $\mathrm{D}^{0}, \mathrm{D}^{+}$and $\mathrm{D}^{*+}$ mesons, and their antiparticles, via their hadronic decay channels $\mathrm{D}^{0} \rightarrow \mathrm{K}^{-} \pi^{+}$(with branching ratio, BR, of $3.88 \pm 0.05 \%$ ), $\mathrm{D}^{+} \rightarrow \mathrm{K}^{-} \pi^{+} \pi^{+}$(BR of $9.13 \pm 0.19 \%$ ), and $\mathrm{D}^{*+} \rightarrow \mathrm{D}^{0} \pi^{+}(\mathrm{BR}$ of $67.7 \pm 0.05 \%)$ with $\mathrm{D}^{0} \rightarrow \mathrm{K}^{-} \pi^{+}[58]$. D-meson candidates were selected with the same strategy as described in [1]. The selection of $\mathrm{D}^{0}$ and $\mathrm{D}^{+}$decays (weak decays with mean proper decay 
length $c \tau \approx 123$ and $312 \mu \mathrm{m}$, respectively [58]) was based on the reconstruction of secondary vertices separated by few hundred microns from the interaction point. In the case of the $\mathrm{D}^{*+}$ strong decay, the decay topology of the produced $\mathrm{D}^{0}$ was reconstructed. $\mathrm{D}^{0}$ and $\mathrm{D}^{+}$candidates were formed using pairs and triplets of tracks with the proper charge sign combination, $|\eta|<0.8, p_{\mathrm{T}}>0.3 \mathrm{GeV} / c$, at least 70 associated space points (out of a maximum of 159) with $\chi^{2} /$ ndf $<2$ of the momentum fit in the TPC, and at least two hits (out of 6) in the ITS, of which at least one had to be in either of the two SPD layers. $\mathrm{D}^{*+}$ candidates were formed by combining $\mathrm{D}^{0}$ candidates with tracks with $p_{\mathrm{T}}>80 \mathrm{MeV} / c$ and at least 3 hits in the ITS, out of which at least one should be in the SPD. The selection of tracks with $|\eta|<0.8$ limits the D-meson acceptance in rapidity. The acceptance drops steeply to zero for $|y|>0.5$ at low $p_{\mathrm{T}}$ and $|y|>0.8$ at $p_{\mathrm{T}}>5 \mathrm{GeV} / c$. A $p_{\mathrm{T}^{-}}$ dependent fiducial acceptance cut, $\left|y_{\mathrm{D}}\right|<y_{\text {fid }}\left(p_{\mathrm{T}}\right)$, was therefore applied on the D-meson rapidity. The cut value, $y_{\text {fid }}\left(p_{\mathrm{T}}\right)$, increases from 0.5 to 0.8 in the transverse momentum range $0<p_{\mathrm{T}}<5 \mathrm{GeV} / c$ according to a second-order polynomial function and it takes a constant value of 0.8 for $p_{\mathrm{T}}>5 \mathrm{GeV} / c$. The selection of the decay topology was based on the displacement of the decay tracks from the interaction vertex, the separation between the secondary and primary vertices, and the pointing angle of the reconstructed D-meson momentum and its flight line from the primary to the secondary vertex. The selections were tuned such that a large statistical significance of the signal and a selection efficiency as high as possible were achieved, which resulted in cut values that depend on the D-meson $p_{\mathrm{T}}$ and species [1]. The same selections were used in all the multiplicity intervals in order to minimise the effect of efficiency corrections in the ratio of the yields. Pion and kaon identification based on the TPC and TOF detectors were used to obtain a further reduction of the background. Cuts in units of resolution (at $\pm 3 \sigma$ ) were applied around the expected mean values of energy deposit $\mathrm{d} E / \mathrm{d} x$ and time-of-flight. Tracks without TOF signal were identified using only the TPC information. Tracks with incompatible TPC and TOF response were considered as non-identified and were used in the analysis as both pion and kaon candidates. Particle identification (PID) was not applied to the pion tracks from the $\mathrm{D}^{*+}$ decay. This selection guarantees a reduction of the background by a factor of about 2 to 3 at low $p_{\mathrm{T}}$, while preserving about $95 \%$ of the signal.

The D-meson raw yields were extracted in each $N_{\text {tracklets }}$ and $p_{\mathrm{T}}$ interval by means of a fit to the candidate invariant mass distributions (mass difference $\Delta M=M(\mathrm{~K} \pi \pi)-M(\mathrm{~K} \pi)$ for $\mathrm{D}^{*+}$ ). Similarly, the multiplicity-integrated raw yields were also evaluated for each $p_{\mathrm{T}}$ interval. The $\mathrm{D}^{0}$ and $\mathrm{D}^{+}$candidate invariant mass distributions were fitted with a function composed of a Gaussian for the signal and an exponential term that describes the background shape. The $\Delta M$ distribution of $\mathrm{D}^{*+}$ candidates, which features a narrow peak at $\Delta M \simeq 145.4 \mathrm{MeV} / c^{2}[58]$, was fitted with a Gaussian function for the signal and a threshold function multiplied by an exponential to model the background $\left(\sqrt{\Delta M-M_{\pi}} \cdot e^{b\left(\Delta M-M_{\pi}\right)}\right)$. The centroids of the Gaussians were found to be compatible with the world-average masses of the D mesons [58] in all multiplicity and $p_{\mathrm{T}}$ intervals. The widths of the Gaussian functions are independent of multiplicity and increase with increasing D-meson $p_{\mathrm{T}}$, ranging between 10 and $20 \mathrm{MeV} / c^{2}$ for $\mathrm{D}^{0}$ and $\mathrm{D}^{+}$and between 600 and $900 \mathrm{keV} / \mathrm{c}^{2}$ for $\mathrm{D}^{*+}$ mesons, consistent with the values obtained in simulations. In order to reduce the 
influence of statistical fluctuations, the raw yields were determined by constraining the D-meson line shape, its mass to the world-average D-meson mass, and its width to the value obtained from a fit to the invariant mass distribution in the multiplicity-integrated sample, where the signal statistical significance is larger. Figure 1 shows the $\mathrm{D}^{0}$ and $\mathrm{D}^{+}$ candidate invariant mass distribution, and $\mathrm{D}^{*+}$ mass difference distributions, for selected $p_{\mathrm{T}}$ and multiplicity intervals. The extraction of the raw signal yields (sum of particle and antiparticle) was possible in five $p_{\mathrm{T}}$ intervals from $1 \mathrm{GeV} / c$ to $20 \mathrm{GeV} / c$ for the $N_{\text {tracklets }}$ ranges reported in table 1 . The analysis covering the range $N_{\text {tracklets }} \in[1,49]$ exploited the minimum-bias triggered sample and was possible for the three D-meson species in three $p_{\mathrm{T}}$ intervals in the range between 2 and $12 \mathrm{GeV} / c$. In addition, the $\mathrm{D}^{0}$ signal was extracted in $N_{\text {tracklets }} \in[1,49]$ for $1<p_{\mathrm{T}}<2 \mathrm{GeV} / c$, and the $\mathrm{D}^{*+}$ signal was determined in three multiplicity intervals for $12<p_{\mathrm{T}}<20 \mathrm{GeV} / c$. The highest multiplicity interval $[50,80]$ was studied with the high multiplicity triggered sample via $\mathrm{D}^{0}$ mesons for $2<p_{\mathrm{T}}<4 \mathrm{GeV} / c$ and the three D-meson species for $4<p_{\mathrm{T}}<8 \mathrm{GeV} / c$. The raw yield extraction in the remaining $p_{\mathrm{T}}$ and multiplicity intervals for the different D-meson species was not performed due to the limited statistics in the analysed data sample and/or the large background.

\subsection{Corrections}

The yields of D mesons were evaluated for each multiplicity and $p_{\mathrm{T}}$ interval starting from the raw counts, $N_{\text {raw }}$, which were divided by the reconstruction, topological and PID selection efficiencies for prompt D mesons, $\varepsilon_{\text {prompt D }}$, and by the number of events analysed in the considered multiplicity interval, $N_{\text {event }}^{j}$. The results are reported as the ratio of yields in each multiplicity interval, $\left(\mathrm{d}^{2} N^{\mathrm{D}^{0}} / \mathrm{d} y \mathrm{~d} p_{\mathrm{T}}\right)^{j}$, to the multiplicity-integrated (average) yield, $\left\langle\mathrm{d}^{2} N^{\mathrm{D}^{0}} / \mathrm{d} y \mathrm{~d} p_{\mathrm{T}}\right\rangle$

$$
\left(\frac{\mathrm{d}^{2} N^{\mathrm{D}^{0}} / \mathrm{d} y \mathrm{~d} p_{\mathrm{T}}}{\left\langle\mathrm{d}^{2} N^{\mathrm{D}^{0}} / \mathrm{d} y \mathrm{~d} p_{\mathrm{T}}\right\rangle}\right)^{j}=\left(\frac{1}{N_{\text {event }}^{j}} \frac{N_{\text {raw D }}^{j}}{\varepsilon_{\text {prompt D }}^{j}}\right) /\left(\frac{1}{N_{\mathrm{MB} \text { trigger }} \varepsilon_{\text {trigger }}} \frac{\left\langle N_{\text {raw D }}\right\rangle}{\left\langle\varepsilon_{\text {prompt D }}\right\rangle}\right),
$$

where the index $j$ identifies the multiplicity interval. The acceptance correction, defined as the fraction of $\mathrm{D}$ mesons within a given rapidity and $p_{\mathrm{T}}$ interval whose decay particles are within the detector coverage, cancels in this ratio. D-meson raw yields have two components: the prompt D-meson contribution, and the feed-down contribution originating from B hadron decays. Equation (4.1) evaluates the yields of prompt D mesons under the assumption that the relative contribution to the D-meson raw yield due to the feed-down from $\mathrm{B}$ hadron decays does not depend on the multiplicity of the event, and is therefore cancelling in the ratio to the multiplicity-integrated values. This assumption is justified by the measurement of the multiplicity dependence of the B-hadron yields, via the non-prompt $\mathrm{J} / \psi$ fraction, presented in section 5 and by PYTHIA simulations. To evaluate the yields per inelastic collisions, the number of events used for the normalisation of the multiplicityintegrated yield has to be corrected for the fraction of inelastic collisions that are not selected by the minimum-bias trigger $N_{\mathrm{MB} \text { trigger }} / \varepsilon_{\text {trigger }}$, with $\varepsilon_{\text {trigger }}=0.85_{-3 \%}^{+6 \%}[56]$. The results are also reported in tables 3 and 5 without this trigger efficiency correction. It was 

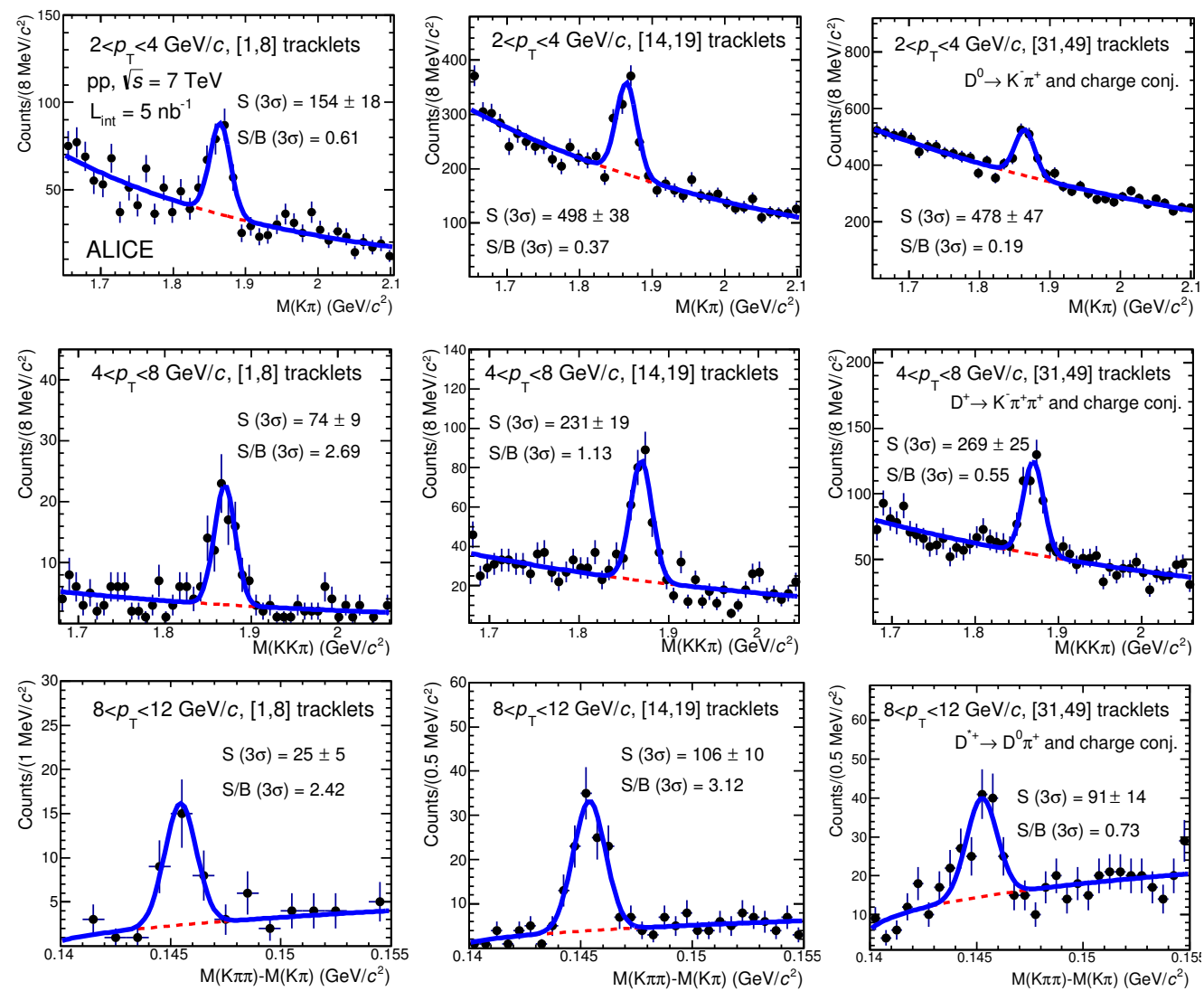

Figure 1. $\mathrm{D}^{0}$ and $\mathrm{D}^{+}$invariant mass and $\mathrm{D}^{*+}$ mass difference distributions for selected $p_{\mathrm{T}}$ and $N_{\text {tracklets }}$ intervals for pp collisions at $\sqrt{s}=7 \mathrm{TeV}$ with $\mathcal{L}_{\text {int }}=5 \mathrm{nb}^{-1}$. The $\mathrm{D}^{0}$ distributions are shown for $2<p_{\mathrm{T}}<4 \mathrm{GeV} / c$ (top-panels), the $\mathrm{D}^{+}$for $4<p_{\mathrm{T}}<8 \mathrm{GeV} / c$ (middle-panels), and the $\mathrm{D}^{*+}$ for $8<p_{\mathrm{T}}<12 \mathrm{GeV} / c$ (bottom-panels). The $N_{\text {tracklets }} \in[1,8],[14,19]$ and [31,49] intervals are shown in the left, middle and right panels respectively. The fits to the candidate invariant mass distributions are also shown.

verified with PYTHIA 6.4.21 [30] Monte Carlo simulations that this minimum-bias trigger is $100 \%$ efficient for $\mathrm{D}$ mesons in the kinematic range of this measurement.

The D-meson efficiency corrections were determined with Monte Carlo simulations using the PYTHIA 6.4.21 event generator [30] with Perugia-0 tune [59], and the GEANT3 transport code [60]. The detector configuration and the LHC beam conditions were included, taking into account their evolution with time during the data taking period. The $\varepsilon_{\text {prompt D }}^{j}$ depends on the $\mathrm{D}$-meson species and on $p_{\mathrm{T}}$. For prompt $\mathrm{D}^{0}$ mesons it is $3-4 \%$ in the $2<p_{\mathrm{T}}<4 \mathrm{GeV} / c$ interval and it increases up to $25-35 \%$ for $p_{\mathrm{T}}>8 \mathrm{GeV} / c$, because less stringent topological selections were used at high $p_{\mathrm{T}}$, where the combinatorial background is smaller. The efficiency for feed-down D mesons is larger by about $20-30 \%$ than for prompt $\mathrm{D}$ mesons. This is due to the fact that feed-down $\mathrm{D}$ mesons decay further away from the interaction vertex and are therefore more efficiently selected by the topological requirements. The D-meson selection efficiency depends also on the multiplicity of charged particles produced in the collision, because the resolution on the position of primary vertex 
improves with increasing multiplicity, providing a better resolution of the variables used for the topological selections. For example, the $\mathrm{D}^{0}$ selection efficiency in $2<p_{\mathrm{T}}<4 \mathrm{GeV} / c$ increases by about $40 \%$ from the lowest to the highest multiplicity intervals considered in this analysis.

\subsection{Systematic uncertainties}

Several sources of systematic uncertainty that could affect the relative yields as expressed in eq. (4.1) were studied. Only the raw yield extraction and the feed-down subtraction contribution were found to have an influence on the relative yields. The influence of the raw signal extraction from the invariant mass distribution was evaluated by using the raw yields obtained with different approaches to separate the signal from the combinatorial background. The contribution to the $\mathrm{D}^{0}$ line shape of mis-identified $\mathrm{K}$ and $\pi$ pairs from $\mathrm{D}^{0}$ decays, e.g. a $\mathrm{D}^{0} \rightarrow \mathrm{K}^{-} \pi^{+}$that passes the selection criteria as $\overline{\mathrm{D}^{0}} \rightarrow \pi^{-} \mathrm{K}^{+}$, was assumed to be the same in all multiplicity intervals and was neglected in this analysis. Different background fit functions were considered (exponential, polynomial, linear for $\mathrm{D}^{0}$ and $\mathrm{D}^{+}$; threshold, $\left(\Delta M-M_{\pi}\right)^{b}$, for $\left.\mathrm{D}^{*+}\right)$; the centroid and width of the Gaussians were left as free parameters in the fit instead of keeping them fixed to the values obtained from the multiplicity-integrated distribution; the raw yield was also extracted by counting the invariant mass histogram entries in a $\pm 3 \sigma$ interval around the peak after subtracting the background evaluated by fitting the distribution side bands (i.e. excluding the $\pm 3 \sigma$ interval around the centroid). The uncertainty was estimated from the stability of the ratio of the raw yields $N_{\text {raw D }}^{j} /\left\langle N_{\text {raw }} \mathrm{D}^{0}\right\rangle$, where the same raw yield extraction method was used in the multiplicity interval $j$ and for the multiplicity-integrated result. The assigned systematic uncertainty varies from $3 \%$ to $15 \%$ depending on the meson species, $p_{\mathrm{T}}$ and multiplicity interval.

The efficiency corrections were calculated independently for each multiplicity interval. The multiplicity distribution of primary charged particles in the Monte Carlo simulation, $P\left(N_{\mathrm{ch}}\right)$, was tuned to reproduce the measured charged-particle multiplicity [57]. The efficiencies obtained with different Monte Carlo setups, that generate different initial multiplicity distributions, showed a good agreement in all multiplicity intervals. This effect was not considered as a source of systematic uncertainty.

The D-meson decay tracks can be included or not in (i) the counting of the number of tracklets, resulting in a shift of the estimated multiplicity, and in (ii) the determination of the primary vertex position, which leads to a different resolution on the vertex position and of the geometrical variables used for the D-meson selection. In the default configuration, the analysis was done excluding the D-meson decay tracks from the primary vertex determination and without excluding them from the multiplicity estimation. To check for possible systematic effects due to the multiplicity determination, the analysis was repeated excluding the D-meson decay tracks from the multiplicity estimation, obtaining compatible results. Furthermore, the relative yields were determined without excluding the D-meson decay tracks from the primary vertex determination. The influence of such variation is properly reproduced by Monte Carlo simulations, leading to a null effect on the 
corrected relative yields. Therefore this effect was not considered as a source of systematic uncertainty.

The analysis was repeated for all D-meson species with different sets of topological selection criteria. It was verified that the corrected relative D-meson yields as defined in eq. (4.1) are not sensitive to this variation. This confirmed that the systematic uncertainty related to the topological selection description in the Monte Carlo cancels in the ratio. The influence of the PID strategy, which is based on the information of TPC and TOF detectors, was studied by also extracting the D-meson raw yields without PID selection criteria, which could be done only for D-meson $p_{\mathrm{T}}>2 \mathrm{GeV} / c$. The ratios of the relative raw yields, $N_{\text {raw D }}^{j} /\left\langle N_{\text {raw D }}{ }^{0}\right\rangle$, with and without PID selections were found to be compatible with unity. As a consequence, this effect was not considered as a source of systematic uncertainty.

As mentioned above, eq. (4.1) describes the prompt corrected yields under the assumption that the fraction of prompt $\mathrm{D}$ mesons, $f_{\text {prompt }}$, does not vary with the event multiplicity. To estimate the uncertainty related to this assumption, the multiplicity integrated $f_{\text {prompt }}$ factor was evaluated with the FONLL B-hadron production cross sections [7], the $\mathrm{B} \rightarrow \mathrm{D}+X$ decay kinematics from EvtGen [61], and the acceptance, reconstruction and selection efficiency of $\mathrm{D}$ mesons from $\mathrm{B}$ decays as described in [1]. The resulting $f_{\text {prompt }}$ values are about $85-95 \%$ depending on the D-meson $p_{\mathrm{T}}$ and the applied selection criteria. The uncertainty due to the $\mathrm{B}$ feed-down contribution to the relative yields, $f_{\mathrm{B}}=1-f_{\text {prompt }}$, was evaluated assuming a linear increase of the fraction $f_{\mathrm{B}}^{j} /\left\langle f_{\mathrm{B}}\right\rangle$ from $1 / 2$ to 2 from the lowest to the highest multiplicity interval. The resulting uncertainties vary with the $p_{\mathrm{T}}$ and multiplicity range and are different for the three mesons. Typical values for intermediate $p_{\mathrm{T}}$ at low multiplicity are ${ }_{-0}^{+5} \%$, and at high multiplicity ${ }_{-20}^{+0} \%$.

\subsection{Results}

The results of the $\mathrm{D}^{0}, \mathrm{D}^{+}$and $\mathrm{D}^{*+}$ meson relative yields for each $p_{\mathrm{T}}$ interval are presented in figures 2 and 3 as a function of the relative charged-particle multiplicity. The relative yields are presented in the top panels with their statistical (vertical bars) and systematic (boxes) uncertainties except the uncertainty on the feed-down fraction, which is drawn separately in the bottom panels in the form of relative uncertainties. The position of the points on the abscissa is the average value of the relative charged-particle multiplicity, $\left(\mathrm{d} N_{\mathrm{ch}} / \mathrm{d} \eta\right) /\left\langle\mathrm{d} N_{\mathrm{ch}} / \mathrm{d} \eta\right\rangle$, for every $N_{\text {tracklets }}$ interval. The $\mathrm{D}^{0}, \mathrm{D}^{+}$and $\mathrm{D}^{*+}$ meson relative yields are compatible in all $p_{\mathrm{T}}$ intervals within uncertainties.

The average of $\mathrm{D}^{0}, \mathrm{D}^{+}$and $\mathrm{D}^{*+}$ relative yields was computed for each $p_{\mathrm{T}}$ interval using as weights the inverse square of their relative statistical uncertainties. The yield extraction uncertainty was considered as uncorrelated systematic uncertainty. The feed-down fraction systematic uncertainty was treated as a correlated systematic uncertainty. The average of the D-meson relative yields for all $p_{\mathrm{T}}$ intervals is summarised in tables 3 and 4 , and presented in figure 4(a). The relative D-meson yields increase with the charged-particle multiplicity by about a factor of 15 in the range between 0.5 and six times $\left\langle\mathrm{d} N_{\mathrm{ch}} / \mathrm{d} \eta\right\rangle$. Figure 4(b) shows the ratios of the average of the D-meson relative yields in various $p_{\mathrm{T}}$ 


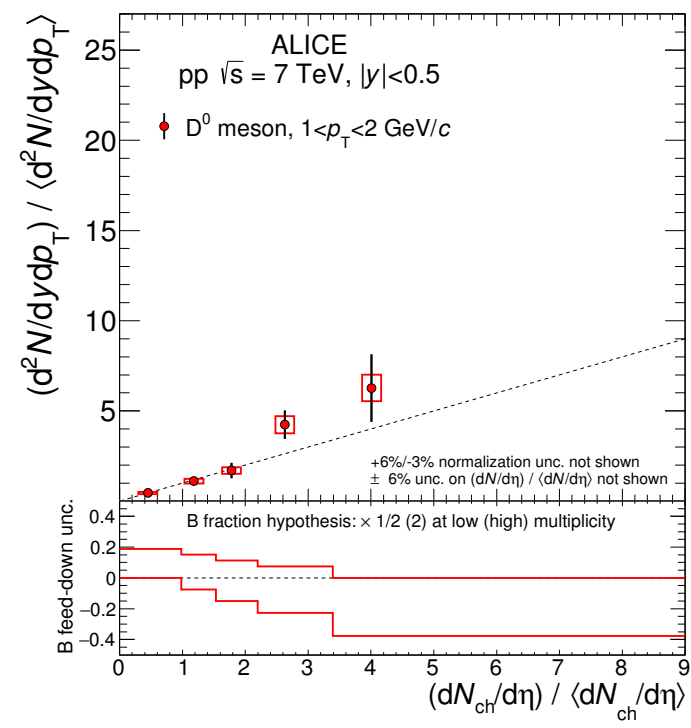

(a) D meson with $1<p_{\mathrm{T}}<2 \mathrm{GeV} / c$.

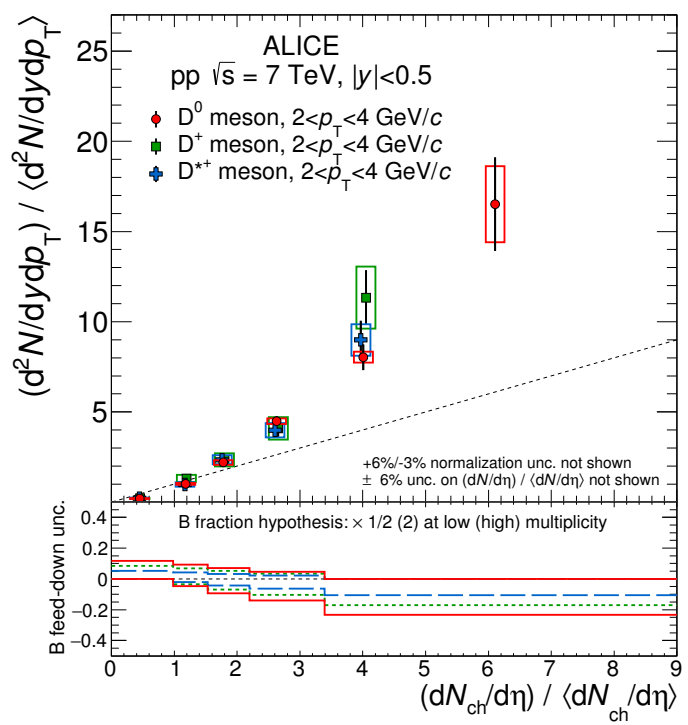

(b) D meson with $2<p_{\mathrm{T}}<4 \mathrm{GeV} / c$.

Figure 2. $\mathrm{D}^{0}, \mathrm{D}^{+}$and $\mathrm{D}^{*+}$ meson relative yields for each $p_{\mathrm{T}}$ interval as a function of chargedparticle multiplicity at central rapidity. The relative yields are presented on the top panels with their statistical (vertical bars) and systematic (boxes) uncertainties, except for the feed-down fraction uncertainty that is drawn separately in the bottom panels. $\mathrm{D}^{0}$ mesons are represented by red circles, $\mathrm{D}^{+}$by green squares, and $\mathrm{D}^{*+}$ by blue triangles. The position of the points on the abscissa is the average value of $\left(\mathrm{d} N_{\mathrm{ch}} / \mathrm{d} \eta\right) /\left\langle\mathrm{d} N_{\mathrm{ch}} / \mathrm{d} \eta\right\rangle$. For $\mathrm{D}^{+}$and $\mathrm{D}^{*+}$ mesons the points are shifted horizontally by $1.5 \%$ to improve the visibility. The diagonal (dashed) line is also shown to guide the eye.

intervals with respect to the $2<p_{\mathrm{T}}<4 \mathrm{GeV} / c$ interval values. The yield enhancement is independent of transverse momentum within the uncertainties of the measurement.

\subsubsection{Studies with the charged-particle multiplicity at forward rapidity}

In the analysis described above, D-meson yields were measured in the same rapidity interval as the charged-particle multiplicity. This could lead to a bias if the particles produced in the charm-quark fragmentation and in the D-meson decay would amount to a large fraction of the measured charged particles. In order to study this possible bias, the measurement of the $\mathrm{D}^{0}$ yields at central rapidity was also performed as a function of the relative charged-particle multiplicity at forward-rapidity. The charge collected by the V0 scintillator counters, covering $-3.7<\eta<-1.7$ and $2.8<\eta<5.1$, was used as multiplicity estimator in this case. The multiplicity value $N_{\mathrm{V} 0}$ was evaluated by dividing the collected charge by the expected average minimum-ionizing-particle charge. The $\mathrm{D}^{0}$ yields were evaluated in intervals of $N_{\mathrm{V} 0}$, and corrected as previously described and summarised in eq. (4.1). The relative yields of $\mathrm{D}^{0}$ mesons are presented in figure 5 as a function of the relative mean multiplicity measured with the V0 counters, $N_{\mathrm{V} 0} /\left\langle N_{\mathrm{V} 0}\right\rangle$. The statistical (systematic) uncertainties are represented by the vertical bars (empty boxes). The systematic uncertainties due to the raw yield extraction and the B feed-down contribution were determined as explained in section 4.3. The uncertainty due to the unknown feed- 


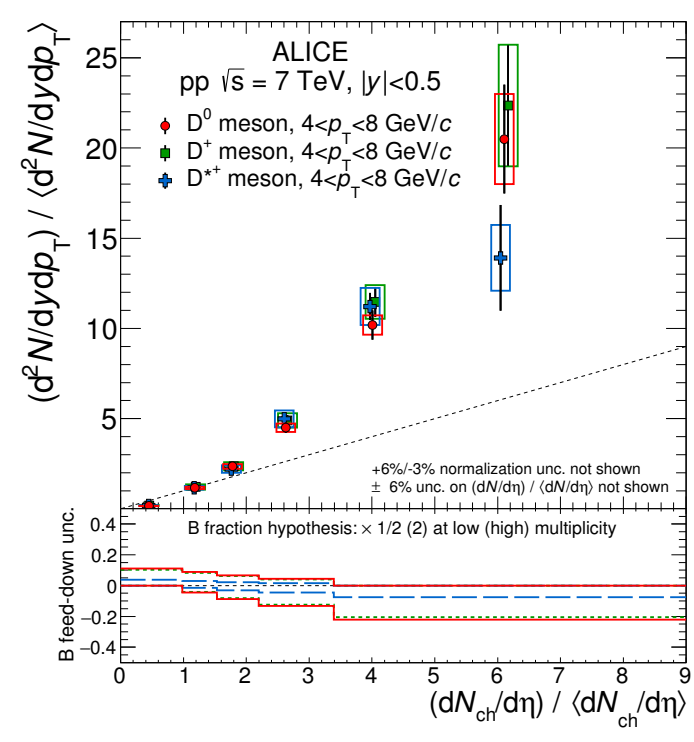

(a) D meson with $4<p_{\mathrm{T}}<8 \mathrm{GeV} / c$.

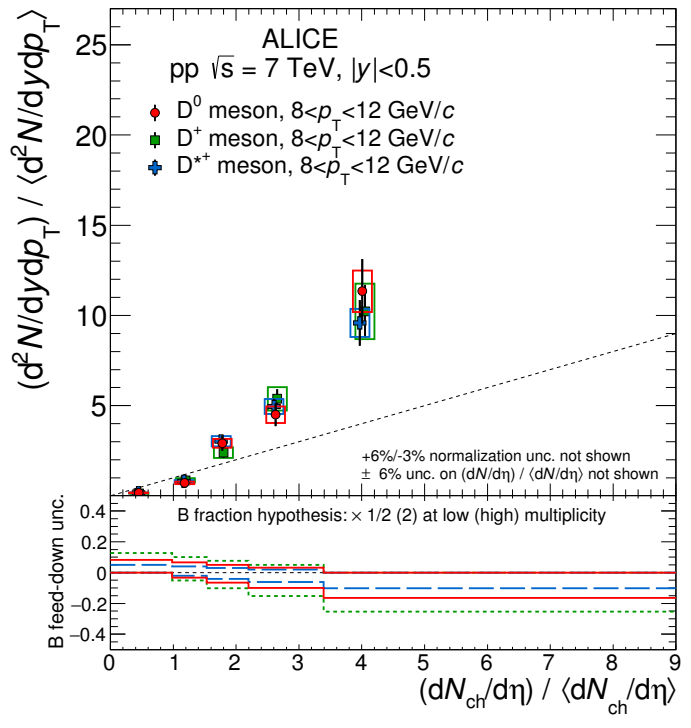

(b) D meson with $8<p_{\mathrm{T}}<12 \mathrm{GeV} / c$.

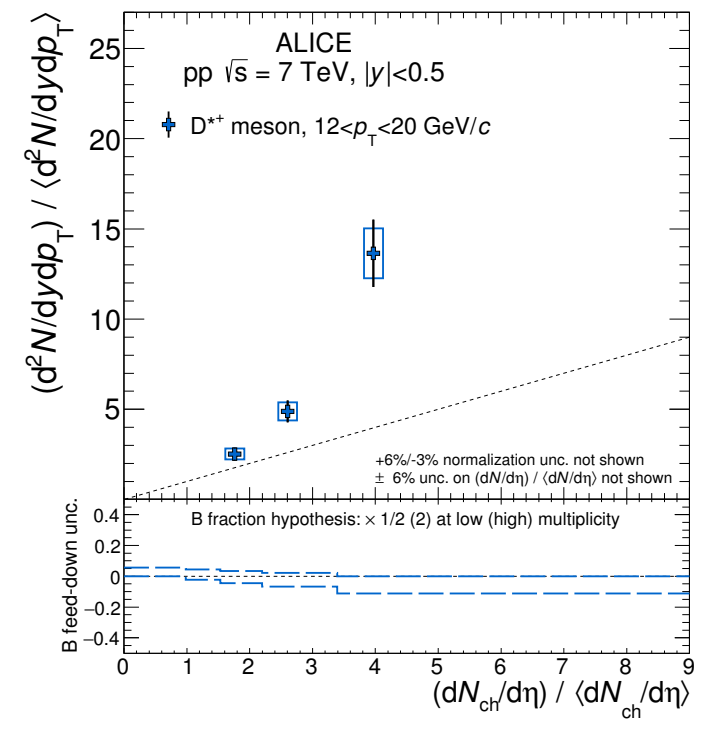

(c) D meson with $12<p_{\mathrm{T}}<20 \mathrm{GeV} / c$.

Figure 3. $\mathrm{D}^{0}, \mathrm{D}^{+}$and $\mathrm{D}^{*+}$ meson relative yields for each $p_{\mathrm{T}}$ interval as a function of chargedparticle multiplicity at central rapidity. The relative yields are presented on the top panels with their statistical (vertical bars) and systematic (boxes) uncertainties, except for the feed-down fraction uncertainty that is drawn separately in the bottom panels. $\mathrm{D}^{0}$ mesons are represented by red circles, $\mathrm{D}^{+}$by green squares, and $\mathrm{D}^{*+}$ by blue triangles. The position of the points on the abscissa is the average value of $\left(\mathrm{d} N_{\mathrm{ch}} / \mathrm{d} \eta\right) /\left\langle\mathrm{d} N_{\mathrm{ch}} / \mathrm{d} \eta\right\rangle$. For $\mathrm{D}^{+}$and $\mathrm{D}^{*+}$ mesons the points are shifted horizontally by $1.5 \%$ to improve the visibility. The diagonal (dashed) line is also shown to guide the eye. 


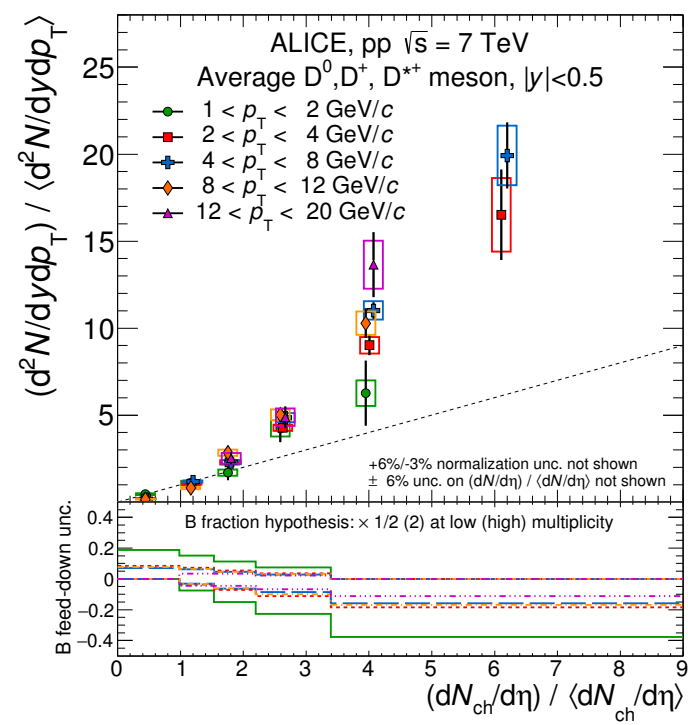

(a) $p_{\mathrm{T}}$ dependence.

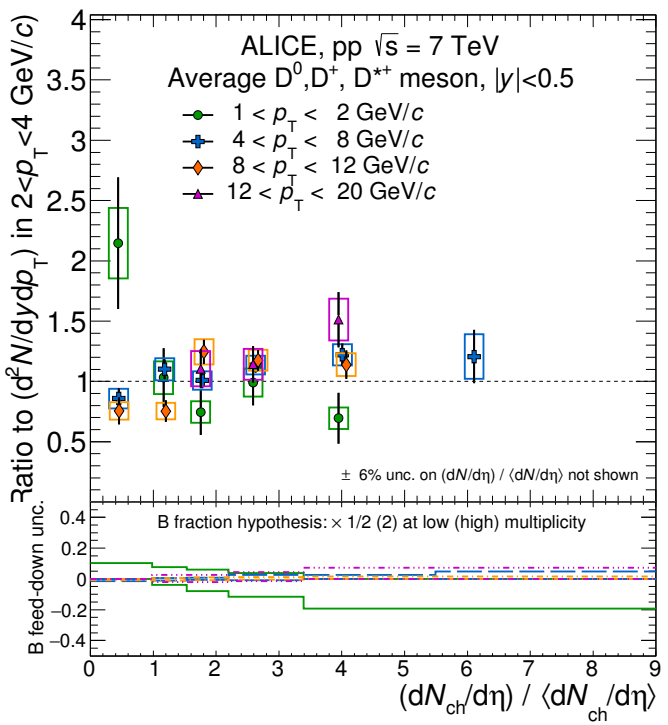

(b) Ratios of $p_{\mathrm{T}}$ intervals vs the $2<p_{\mathrm{T}}<4 \mathrm{GeV} / c$.

Figure 4. Average of $\mathrm{D}^{0}, \mathrm{D}^{+}$and $\mathrm{D}^{*+}$ relative yields as a function of the relative chargedparticle multiplicity at central rapidity. (a) Average of D-meson relative yields in $p_{\mathrm{T}}$ intervals. (b) Ratio of the average relative yields in all $p_{\mathrm{T}}$ intervals with respect to that of the $2<p_{\mathrm{T}}<$ $4 \mathrm{GeV} / c$ interval. The results are presented in the top panels with their statistical (vertical bars) and systematic (boxes) uncertainties, except for the feed-down fraction uncertainty that is drawn separately in the bottom panels. The position of the points on the abscissa is the average value of $\left(\mathrm{d} N_{\mathrm{ch}} / \mathrm{d} \eta\right) /\left\langle\mathrm{d} N_{\mathrm{ch}} / \mathrm{d} \eta\right\rangle$. For some $p_{\mathrm{T}}$ intervals the points are shifted horizontally by $1.5 \%$ to improve the visibility. The dashed lines are also shown to guide the eye, a diagonal on (a) and a constant on (b).

down fraction evolution with the charged-particle multiplicity is drawn separately in the bottom panels. The points are located on the $x$-axis at the average value of the relative mean multiplicity, $N_{\mathrm{V} 0} /\left\langle N_{\mathrm{V} 0}\right\rangle$. The uncertainty on the mean multiplicity values, $N_{\mathrm{V} 0}$, was determined by comparing the mean and median values of the distributions. It was found to be below $3 \%$ for each multiplicity interval, and about $24 \%$ for the multiplicity integrated value. The uncertainty on $N_{\mathrm{V} 0} /\left\langle N_{\mathrm{V} 0}\right\rangle$ is not displayed on this figure. These results are also summarised in tables 5 and 6 . The $\mathrm{D}^{0}$ relative yields increase with the relative uncorrected multiplicity at forward rapidity, as measured with the V0 detector. The results in the $2<p_{\mathrm{T}}<4 \mathrm{GeV} / c$ and $4<p_{\mathrm{T}}<8 \mathrm{GeV} / c$ intervals are compatible within uncertainties. The results with the V0 multiplicity estimator indicate that the increase of the D-meson yield with the event multiplicity observed with the mid-rapidity estimator is not related to the fact that charmed mesons, originating from the fragmentation of charm quarks produced in hard partonic scattering processes, and the charged particle multiplicity are measured in the same pseudo-rapidity range. A qualitatively similar increasing trend of D-meson yield with multiplicity is indeed observed also when an $\eta$ gap is introduced between the regions where the D-mesons and the multiplicity are measured. 


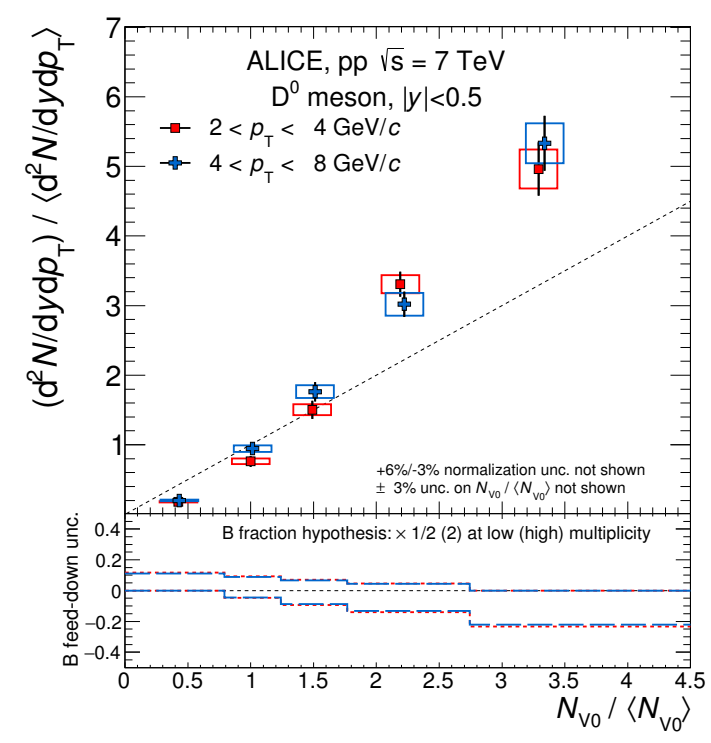

Figure 5. $\mathrm{D}^{0}$ meson relative yields at $|y|<0.5$ for two $p_{\mathrm{T}}$ intervals as a function of the relative charged-particle multiplicity, $N_{\mathrm{V} 0}$, measured at $-3.7<\eta<-1.7$ and $2.8<\eta<5.1$. The relative yields are presented on the top panels with their statistical (vertical bars) and systematic (boxes) uncertainties, except the uncertainty on the feed-down fraction which is drawn separately in the bottom panels. The position of the points on the abscissa is at the average value of $N_{\mathrm{V} 0} /\left\langle N_{\mathrm{V} 0}\right\rangle$, shifted by $1.5 \%$ to improve the visibility. The diagonal (dashed) line is also shown to guide the eye.

\section{$5 \quad$ Non-prompt $\mathrm{J} / \psi$ analysis}

\section{$5.1 \quad$ Non-prompt $\mathrm{J} / \psi$ reconstruction}

The fraction of non-prompt $\mathrm{J} / \psi$ in the inclusive $\mathrm{J} / \psi$ yields, $f_{\mathrm{B}}$, was measured as a function of the charged-particle multiplicity by studying displaced $\mathrm{J} / \psi$ mesons that decay into electron pairs in the rapidity range $|y|<0.9$. This measurement, combined with the inclusive $\mathrm{J} / \psi$ relative yield [40], provides the multiplicity dependence of the production of beauty hadrons. $\mathrm{J} / \psi$ candidates were formed by combining pairs of opposite-sign electron tracks. The tracks were required to have $p_{\mathrm{T}}>1 \mathrm{GeV} / c$, at least 70 (out of a maximum of 159) associated space points in the TPC with a $\chi^{2} /$ ndf of the momentum fit lower than 2 , and to point back to the primary interaction vertex within $1 \mathrm{~cm}$ in the transverse plane. The tracks were also required to have at least one associated hit in the SPD detector, with the constraint that one of the two tracks should have a hit in the first SPD layer. Electron identification was based only on the TPC information. A selection of $\pm 3 \sigma$ around the expected mean values of the specific energy deposit $\mathrm{d} E / \mathrm{d} x$ for electrons was used. To further reduce the background, a $\pm 3.5 \sigma( \pm 3 \sigma)$ exclusion band around the expected mean specific energy deposit for pions (protons) was also applied. In order to reduce the combinatorial background, electron candidates compatible, together with a positron candidate, with being products of $\gamma$-conversions (invariant mass below $100 \mathrm{MeV} / c^{2}$ ) were removed. 
The measurement of $f_{\mathrm{B}}$ is based on a statistical discrimination of $\mathrm{J} / \psi$ mesons produced at a secondary vertex displaced from the primary pp collision vertex. The signed projection of the $\mathrm{J} / \psi$ flight distance onto its transverse momentum vector, $\overrightarrow{p_{\mathrm{T}}}$, was constructed as $L_{\mathrm{xy}}=\left(\vec{L} \cdot \overrightarrow{p_{\mathrm{T}}}\right) / p_{\mathrm{T}}$, where $\vec{L}$ is the vector from the primary vertex to the $\mathrm{J} / \psi$ decay vertex. The pseudo-proper decay length $x=\left(c \cdot L_{\mathrm{xy}} \cdot m\right) / p_{\mathrm{T}}$ was calculated from the observed decay length using the world-average $\mathrm{J} / \psi$ mass $m(\mathrm{~J} / \psi)=3096.916 \pm 0.011 \mathrm{MeV} / c^{2}$ [58]. The fraction of non-prompt $\mathrm{J} / \psi$ can be determined from a 2-dimensional un-binned loglikelihood fit to $x$ and the unlike-sign di-electron invariant mass distributions. The fit procedure and the functions used to describe the invariant mass and the pseudo-proper decay length distributions were introduced in [10].

The fraction of non-prompt $\mathrm{J} / \psi$ as a function of the relative charged-particle multiplicity was determined for $p_{\mathrm{T}}>1.3 \mathrm{GeV} / c$ in five multiplicity intervals in the $N_{\text {tracklets }}$ range $[4,49]$. The $N_{\text {tracklets }} \in[1,3]$ range was excluded from this analysis due to the poor pseudo-proper decay length resolution, $R(x)$, and the presence of a bias in the determination of $x$ in the case of non-prompt candidates. The resolution of the pseudo-proper decay length is determined with Monte Carlo simulations evaluating the RMS of the $x$ distributions of reconstructed promptly produced $\mathrm{J} / \psi$ mesons. The event primary vertex can be computed with or without removing the decay tracks of the $\mathrm{J} / \psi$ candidates. The removal of the decay tracks causes a degradation of the resolution on $x$, especially in the low-multiplicity intervals, as a consequence of the lower precision in the determination of the primary vertex with a reduced number of tracks. For simulated events with non-prompt $\mathrm{J} / \psi$, the removal of the decay tracks also results in a shift of the primary vertex position away from the secondary decay vertex of the beauty hadrons, which is reflected in a systematic shift of the mean of the $x$ distribution. However, one should consider that beauty quarks are always produced in pairs: the two decay tracks from the non-prompt-J $/ \psi$, when included, pull the primary vertex towards the beauty hadron decay vertex, but the charged tracks from the decay of the second beauty quark, which enter in the barrel acceptance, pull the primary vertex in the opposite direction. The shift is larger in the lowest multiplicity bin where it reaches about $35 \mu \mathrm{m}$. This bias is reduced when the $\mathrm{J} / \psi$ decay tracks are kept in the evaluation of the primary vertex. The effect of the bias, estimated with Monte Carlo simulations, is a reduction ${ }^{1}$ of the measured $f_{\mathrm{B}}$ by about $20 \%$ for events with $N_{\text {tracklets }}=4$, and it becomes negligible for $N_{\text {tracklets }}>10$. Therefore, the primary vertex was computed considering all reconstructed tracks. To correct for the remaining bias, a modification in the resolution function, $R(x)$, used to describe the non-prompt $\mathrm{J} / \psi$ in the

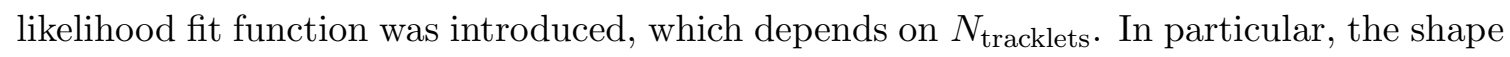
of the resolution function was adjusted to obtain a good matching between the function used to describe the non-prompt $\mathrm{J} / \psi$ in the likelihood fit (a convolution of a template of the $x$ distribution of $\mathrm{J} / \psi$ from beauty hadron decays with the resolution function [10]) and the pseudo-proper decay length distribution of reconstructed secondary $\mathrm{J} / \psi$ from Monte Carlo simulations.

\footnotetext{
${ }^{1}$ This shift would be greater than $50 \mu \mathrm{m}$ in the $N_{\text {tracklets }}$ interval $[1,3]$, leading to a large bias on the extracted $f_{\mathrm{B}}$ value (up to $35 \%$ ). The correction for this bias would introduce a large systematic uncertainty.
} 

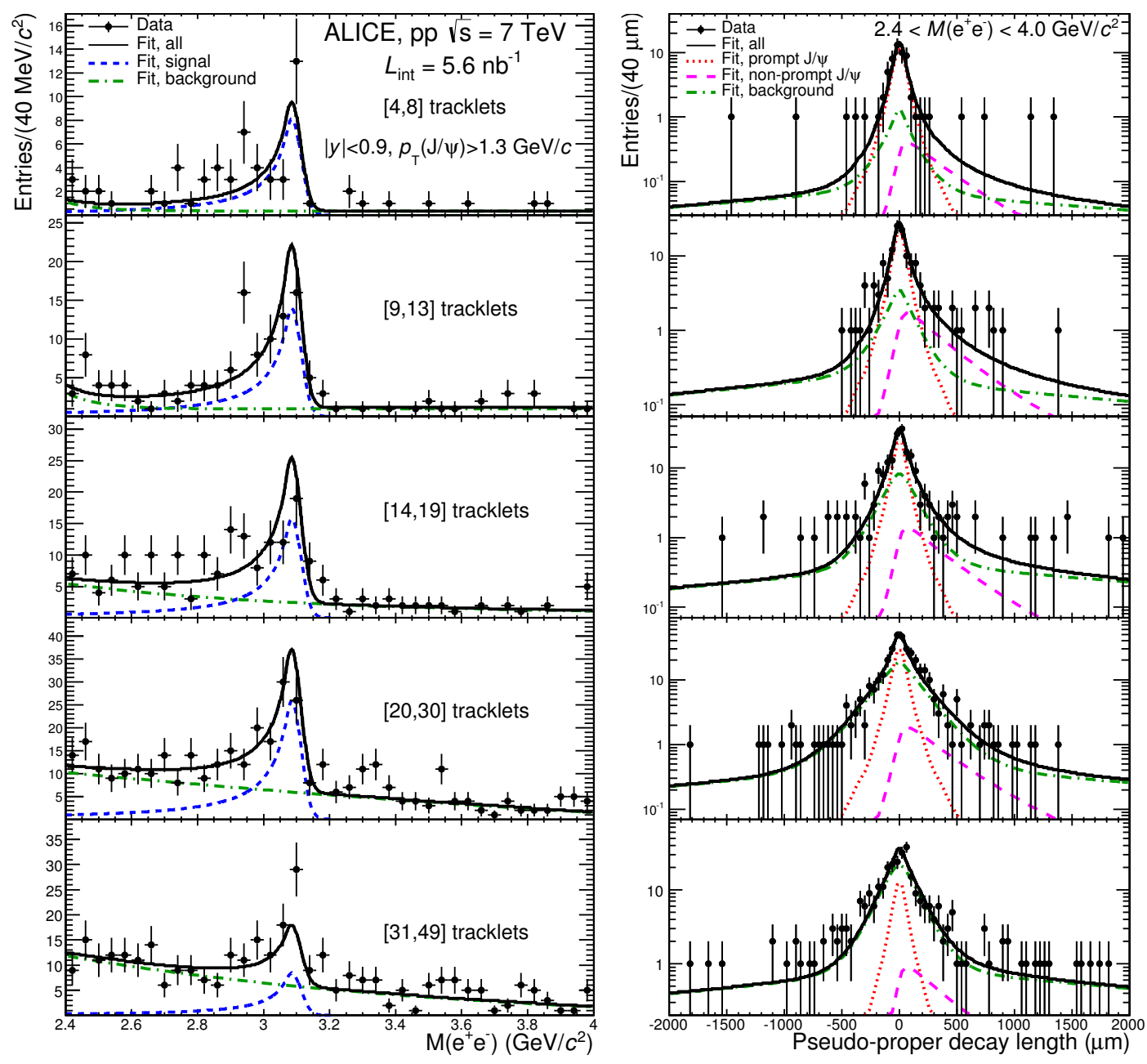

Figure 6. $\mathrm{J} / \psi$ invariant mass and pseudo-proper decay length distributions in several multiplicity intervals with superimposed the likelihood fit results. The contributions of the signal, the background and their sum are represented with dashed, dot-dashed and full lines, respectively. In addition, the pseudo-proper decay length figures include the prompt and non-prompt contributions to the inclusive yields with dotted and long-dashed lines.

Figure 6 presents the invariant mass and pseudo-proper decay length distributions for $p_{\mathrm{T}}>1.3 \mathrm{GeV} / c$ for each multiplicity interval together with a projection of the result of the log-likelihood fit.

\subsection{Corrections}

For all multiplicity intervals, the measured fraction of non-prompt $\mathrm{J} / \psi, f_{\mathrm{B}}^{\prime}$, was corrected using the acceptance and reconstruction efficiency of prompt, $\langle A c c \times \varepsilon\rangle_{\text {prompt }}$, and nonprompt $\mathrm{J} / \psi,\langle A c c \times \varepsilon\rangle_{\mathrm{B}}$, as

$$
f_{\mathrm{B}}=\left(1+\frac{1-f_{\mathrm{B}}^{\prime}}{f_{\mathrm{B}}^{\prime}} \cdot \frac{\langle A c c \times \varepsilon\rangle_{\mathrm{B}}}{\langle A c c \times \varepsilon\rangle_{\text {prompt }}}\right)^{-1} .
$$


Here all terms refer to non-prompt $\mathrm{J} / \psi$ with $p_{\mathrm{T}}>1.3 \mathrm{GeV} / c$. The corrections for acceptance and efficiency were computed using Monte Carlo simulations using the GEANT3 transport code [60]. Prompt $\mathrm{J} / \psi$ were generated with a $p_{\mathrm{T}}$ distribution extrapolated from CDF measurements [16] and a $y$ distribution parameterised with the Colour Evaporation Model (CEM) [62, 63]. Beauty hadrons were generated using the PYTHIA 6.4.21 event generator [30] with Perugia-0 tune [64]. The acceptance times efficiency values for prompt and non-prompt $\mathrm{J} / \psi$ have a minimum of $8 \%$ at $p_{\mathrm{T}}=2 \mathrm{GeV} / c$ and a broad maximum of $12 \%$ at $p_{\mathrm{T}}=7 \mathrm{GeV} / c$ [65]. The relative difference in efficiency between prompt and non-prompt $\mathrm{J} / \psi$ is only about $3 \%$. The ratio $\langle A c c \times \varepsilon\rangle_{\mathrm{B}} /\langle A c c \times \varepsilon\rangle_{\text {prompt }}$ is assumed to be independent of multiplicity. The uncertainty related to this assumption is discussed in the next section.

The measured non-prompt $\mathrm{J} / \psi$ fractions were extrapolated from $p_{\mathrm{T}}>1.3 \mathrm{GeV} / c$ down to $p_{\mathrm{T}}=0$ using

$$
f_{\mathrm{B}}^{\text {extr }}\left(p_{\mathrm{T}}>0\right)=\alpha^{\text {extr }} \cdot f_{\mathrm{B}}\left(p_{\mathrm{T}}>1.3 \mathrm{GeV} / c\right) ; \quad \alpha^{\text {extr }}=\frac{f_{\mathrm{B}}^{\text {model }}\left(p_{\mathrm{T}}>0\right)}{f_{\mathrm{B}}^{\text {model }}\left(p_{\mathrm{T}}>1.3 \mathrm{GeV} / c\right)},
$$

where $f_{\mathrm{B}}^{\text {model }}$ represents a functional form modelled on existing data. It was calculated as the ratio of the differential cross section of non-prompt $\mathrm{J} / \psi$, as obtained with FONLL calculations [7], to that of inclusive $\mathrm{J} / \psi$, parameterised by the phenomenological function defined in [66]:

$$
f_{\mathrm{B}}^{\text {model }}\left(p_{\mathrm{T}}\right)=\frac{\mathrm{d}^{2} \sigma_{\mathrm{J} / \psi \leftarrow h_{B}}^{\mathrm{FONLL}}}{\mathrm{d} y \mathrm{~d} p_{\mathrm{T}}} / \frac{\mathrm{d}^{2} \sigma_{\mathrm{J} / \psi}^{\text {phenom }}}{\mathrm{d} y \mathrm{~d} p_{\mathrm{T}}} .
$$

A combined fit to the existing results of $f_{\mathrm{B}}$ in pp collisions at $7 \mathrm{TeV}[10,13,67,68]$ in the rapidity bin closest to central rapidity was performed to determine the parameters of the phenomenological parameterisation. The extrapolation factor obtained is $\alpha^{\text {extr }}=0.99_{-0.03}^{+0.01}$. Its uncertainties were determined by repeating the fit by (i) excluding the $\mathrm{LHCb}$ data points at forward rapidities, and (ii) using for the non-prompt $\mathrm{J} / \psi$ cross section the upper and lower uncertainty bands of the FONLL predictions, obtained by varying the factorisation and renormalisation scales, instead of the central values. The uncertainties were determined by the maximum and minimum $\alpha^{\text {extr }}$ values obtained from these fit variations. The $f_{\mathrm{B}}$ fractions in all multiplicity intervals were extrapolated using the same $\alpha^{\text {extr }}$ value, evaluated from the fit of the multiplicity integrated measurements.

\subsection{Systematic uncertainties}

The systematic uncertainty introduced by the experimental resolution on the primary vertex position was evaluated by repeating the fitting procedure in two alternative ways: (i) the primary vertex was evaluated without removing the decay tracks of the $\mathrm{J} / \psi$ candidates. The fit was performed using the standard resolution function for non-prompt $\mathrm{J} / \psi$, that does not depend on multiplicity, but the $x$ distribution of the non-prompt $\mathrm{J} / \psi$ was shifted by a multiplicity-dependent value, which was determined by the Monte Carlo simulation. (ii) The event primary vertex was computed after removing the decay tracks of the $\mathrm{J} / \psi$ candidates and the fit was performed using the corresponding degraded resolution 
function $R(x)$ and without any shift. The resulting uncertainties decrease with increasing multiplicity, ranging from $19 \%$ in the lowest multiplicity interval to $3 \%$ at the highest multiplicities.

The uncertainty related to the extrapolation of $f_{\mathrm{B}}$ from $p_{\mathrm{T}}>1.3 \mathrm{GeV} / c$ to $p_{\mathrm{T}}>0$ was estimated with the method discussed above and it is about $3 \%$. This uncertainty was assumed to be uncorrelated among the multiplicity intervals.

The resolution function used in the fits is based on Monte Carlo simulations, which might introduce systematic effects. These were estimated by repeating the log-likelihood fits modifying the resolution function, $R(x)$, according to $(1 /(1+\delta)) \cdot R(x /(1+\delta))$, where $\delta$ is the relative variation of the RMS of the resolution function, and it was varied from -0.1 to +0.1 to take into account the uncertainties in the Monte Carlo description. The systematic uncertainty due to the resolution function increases with multiplicity from $8 \%$ to $20 \%$.

The $p_{\mathrm{T}}$ distribution of the signal candidates (prompt and non-prompt $\mathrm{J} / \psi$ ) could depend on the event multiplicity which could affect the shape of the resolution function which depends on the $\mathrm{J} / \psi p_{\mathrm{T}}$. The average $p_{\mathrm{T}}$ of the signal candidates was estimated from data in each multiplicity interval and found to be constant as a function of event multiplicity within statistical uncertainties about $\pm 10 \%$. The influence of a $\left\langle p_{\mathrm{T}}\right\rangle$ variation on the resolution function was determined using Monte Carlo simulations: the $p_{\mathrm{T}}$ distribution was changed, considering softer or harder $p_{\mathrm{T}}$ distributions, in order to obtain a $\pm 10 \%$ variation of the $\left\langle p_{\mathrm{T}}\right\rangle$. The corresponding variations obtained for the RMS of the resolution function are $+7 \%$ and $-8.5 \%$ for the softer and harder $p_{\mathrm{T}}$ distribution, respectively. The latter variations are within those quoted for the resolution function $( \pm 10 \%)$, therefore no additional uncertainty was included.

The acceptance times efficiency values of prompt and non-prompt $\mathrm{J} / \psi$ reconstructed for $p_{\mathrm{T}}>1.3 \mathrm{GeV} / c$ are of the order of $10 \%$ and differ by $3 \%$. The influence of the $p_{\mathrm{T}}$ shape assumed in the simulation on the ratio $\langle A c c \times \varepsilon\rangle_{\mathrm{B}} /\langle A c c \times \varepsilon\rangle_{\text {prompt }}$ was evaluated by varying the average $p_{\mathrm{T}}$ of the simulated $\mathrm{J} / \psi$ distributions within $\pm 50 \%$. A $1 \%$ variation in the acceptance was obtained both for prompt and non-prompt $\mathrm{J} / \psi$. The corresponding variation obtained on $f_{\mathrm{B}}$ through the eq. (5.1) is about $1 \%$.

The pseudo-proper decay length shape of the combinatorial background was determined by a fit to the $x$ distribution of the candidates in the sidebands of the invariant mass [10]. By varying the fit parameters within their errors an envelope of distributions was obtained, whose extremes were used in the likelihood fit to estimate the systematic uncertainty. It increases slightly with multiplicity, ranging from $1 \%$ to $5 \%$.

The uncertainty on the background invariant mass shape, which was determined by fits to the invariant mass distributions of opposite-sign candidates in each multiplicity bin, was evaluated by using like-sign distributions instead, adopting the same procedure as described in [10]. The systematic uncertainty is about $7 \%$, independent of the chargedparticle multiplicity.

The shape of the $x$ distribution of $\mathrm{J} / \psi$ from b-hadrons was evaluated using PYTHIA 6.4.21 [30]. The systematic uncertainty on its shape was computed by (i) changing the b-hadron decay kinematic, using EvtGen [61] instead of PYTHA 6.4.21 or (ii) by assuming 
a harder and a softer b-hadron $p_{\mathrm{T}}$ distribution, resulting in a $\left\langle p_{\mathrm{T}}\right\rangle$ variation of about $\pm 15 \%$. The resulting systematic uncertainty is about $3 \%$, constant with multiplicity.

The signal invariant mass shape was fixed from the Monte Carlo simulation which includes the detector resolution effects and the radiative decays using the EvtGen [61] package. The effect on the invariant mass signal shape due to the uncertainty on the detector material was studied with dedicated Monte Carlo simulations, where the detector material budget was varied by $\pm 6 \%$ with respect to the nominal values [69, 70]. The resulting systematic uncertainty on $f_{\mathrm{B}}$ is $3 \%$ in the lowest event multiplicity interval and $5 \%$ in the highest one.

The systematic uncertainties on the pseudo-proper decay length of the combinatorial background, on the $p_{\mathrm{T}^{-}}$-extrapolation uncertainty $\alpha^{\text {extr }}$ and on the invariant mass shape of background are found or, in the case of $\alpha^{\text {extr }}$, assumed to be uncorrelated among multiplicity intervals. The remaining systematic uncertainties are (fully or partially) correlated in different multiplicity intervals.

\subsection{Results}

The relative yield of $\mathrm{J} / \psi$ from beauty hadron decays as a function of the charged-particle multiplicity was evaluated from the inclusive $\mathrm{J} / \psi$ yield and the fraction of non-prompt $\mathrm{J} / \psi$ per multiplicity interval:

$$
\frac{\mathrm{d} N_{\mathrm{J} / \psi}^{\text {non-prompt }} / \mathrm{d} y}{\left\langle\mathrm{~d} N_{\mathrm{J} / \psi}^{\text {non-prompt }} / \mathrm{d} y\right\rangle}=\frac{\mathrm{d} N_{\mathrm{J} / \psi} / \mathrm{d} y}{\left\langle\mathrm{~d} N_{\mathrm{J} / \psi} / \mathrm{d} y\right\rangle} \cdot \frac{f_{\mathrm{B}}}{\left\langle f_{\mathrm{B}}\right\rangle} .
$$

$f_{\mathrm{B}}$ is the fraction of non-prompt $\mathrm{J} / \psi$ in each multiplicity interval, $\left\langle f_{\mathrm{B}}\right\rangle$ is the fraction in the multiplicity integrated sample [10], and $\left(\mathrm{d} N_{\mathrm{J} / \psi} / \mathrm{d} y\right) /\left\langle\mathrm{d} N_{\mathrm{J} / \psi} / \mathrm{d} y\right\rangle$ is the inclusive $\mathrm{J} / \psi$ relative yield measured for $p_{\mathrm{T}}>0$ in each multiplicity interval normalized to its value in inelastic pp collisions [40]. All these quantities were measured using the same data sample and the statistical correlations were taken into account. In the first charged-particle multiplicity class $N_{\text {tracklets }} \in[4,8]$, which is used for the non-prompt $\mathrm{J} / \psi$ analysis presented here, the relative yield of inclusive $\mathrm{J} / \psi$ normalized to the inelastic cross section is $\left(\mathrm{d} N_{\mathrm{J} / \psi} / \mathrm{d} y\right) /\left\langle\mathrm{d} N_{\mathrm{J} / \psi} / \mathrm{d} y\right\rangle=0.41 \pm 0.07$ (stat) \pm 0.01 (syst). The values of $f_{\mathrm{B}}$ extrapolated to $p_{\mathrm{T}}>0$ were used in eq. (5.4), providing the non-prompt $\mathrm{J} / \psi$ relative yields for $p_{\mathrm{T}}>0$. The relative yields of inclusive $\mathrm{J} / \psi$ were also recomputed for $p_{\mathrm{T}}>1.3 \mathrm{GeV} / c$ and no difference was observed with respect to those for $p_{\mathrm{T}}>0$ within the uncertainties.

The results for the fraction of non-prompt $\mathrm{J} / \psi$ for both $p_{\mathrm{T}}>0$ and $p_{\mathrm{T}}>1.3 \mathrm{GeV} / c$, the relative yields of prompt and non-prompt $\mathrm{J} / \psi$ in each multiplicity bin for $p_{\mathrm{T}}>0$ are summarized in tables 7 and 8 and shown in figure 7 .

\section{Comparison of charm and beauty production}

Figure $8(\mathrm{a})$ presents prompt $\mathrm{D}$ meson and inclusive $\mathrm{J} / \psi$ results to compare open and hidden charm production. The average prompt D-meson results are shown in the $2<$ 


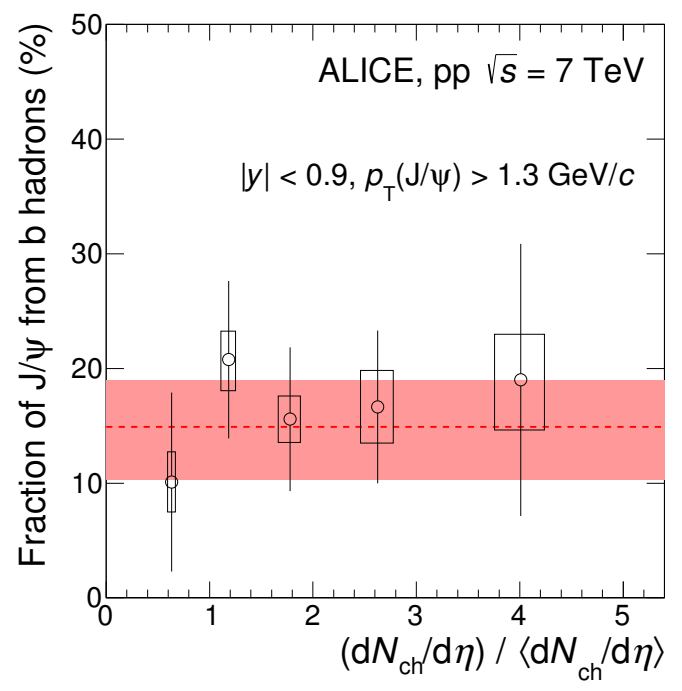

Figure 7. Non-prompt $\mathrm{J} / \psi$ fraction as a function of the relative charged-particle multiplicity at central rapidity for $p_{\mathrm{T}}>1.3 \mathrm{GeV} / c$. The vertical bars represent the statistical uncertainties, while the empty boxes stand for the systematic uncertainties. The width and the height of these empty boxes indicate the measurement uncertainty on the horizontal and vertical axis respectively. The dashed line shows the value of $f_{\mathrm{B}}$ measured in the same $p_{\mathrm{T}}$ range and integrated over multiplicity [10]. The shaded area represents the statistical and systematic uncertainties on the multiplicity-integrated result added in quadrature.

$p_{\mathrm{T}}<4 \mathrm{GeV} / c$ interval with the $p_{\mathrm{T}}$-integrated inclusive $\mathrm{J} / \psi$ measurement ${ }^{2}$ at central and forward-rapidity by the ALICE experiment [40]. The results for prompt $\mathrm{J} / \psi$ at central rapidity from this paper $\left(p_{\mathrm{T}}>0\right)$ and for prompt $\mathrm{D}$ mesons $\left(2<p_{\mathrm{T}}<4 \mathrm{GeV} / c\right)$ are compared in figure 8(b). A similar increase of the relative yield with the charged-particle multiplicity is observed for open and hidden charm production both at central and forward rapidities.

Figure 8(c) superimposes the open charm and beauty production measurements reported in this paper showing the average prompt D-meson results in the $2<p_{\mathrm{T}}<4 \mathrm{GeV} / c$ interval and the $p_{\mathrm{T}}$-integrated non-prompt $\mathrm{J} / \psi$ measurement at central rapidity. The results are compatible within the measurement uncertainties.

Open charm, open beauty and hidden charm hadron relative yields present a similar increase with charged-particle multiplicity. The comparison of open and hidden heavy flavour production suggests that this behaviour is most likely related to the $c \overline{\mathrm{c}}$ and $\mathrm{b} \overline{\mathrm{b}}$ production processes, and is not significantly influenced by hadronisation. The enhancement of the heavy-flavour relative yields with the charged-particle multiplicity is qualitatively consistent with the calculations of the contribution from MPIs to particle production at LHC

\footnotetext{
${ }^{2}$ After the inclusive $\mathrm{J} / \psi$ measurement was published in reference [40], there was an improvement of the ALICE measurement of the inelastic cross section in pp collisions at $\sqrt{s}=7 \mathrm{TeV}$. The improved evaluation of the inelastic cross section does not rely on Monte Carlo, hence the systematic uncertainty is larger [56]. To allow a proper comparison with the results reported here, we updated the published inclusive $\mathrm{J} / \psi$ measurement by the corresponding change of the trigger efficiency for inelastic collisions $0.864 / 0.85$. The normalisation uncertainties were also changed from $1.5 \%$ to ${ }_{-3}^{+6} \%$.
} 


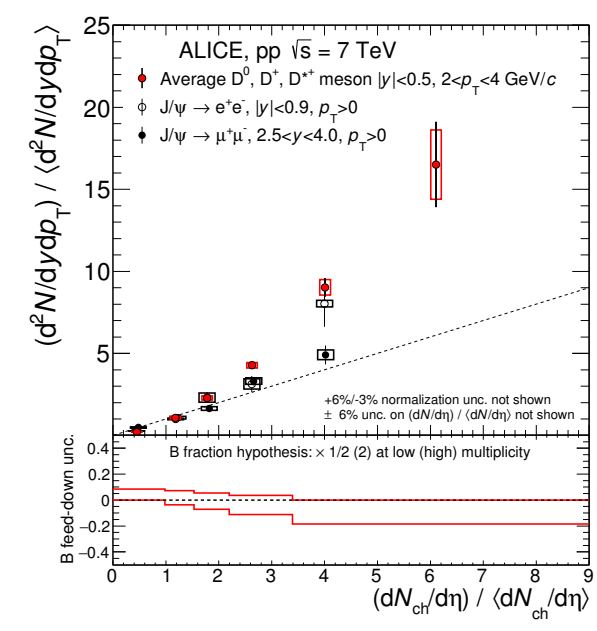

(a) D meson with $2<p_{\mathrm{T}}<4 \mathrm{GeV} / c$ and inclusive $\mathrm{J} / \psi$.

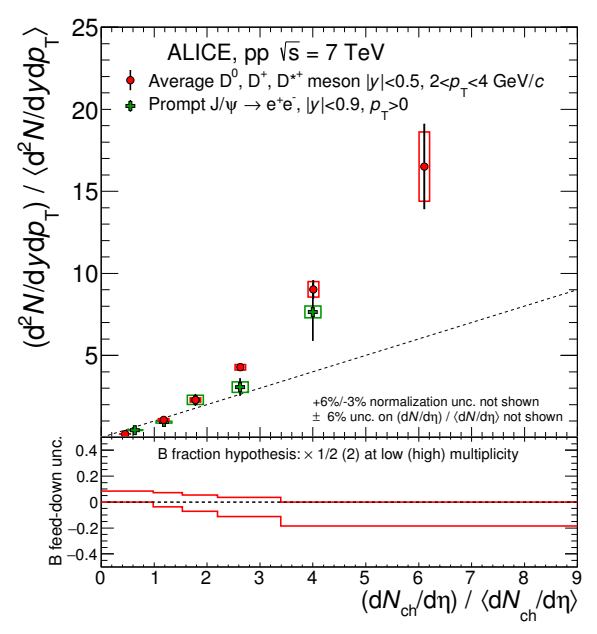

(b) D meson with $2<p_{\mathrm{T}}<4 \mathrm{GeV} / c$ and prompt $\mathrm{J} / \psi$.

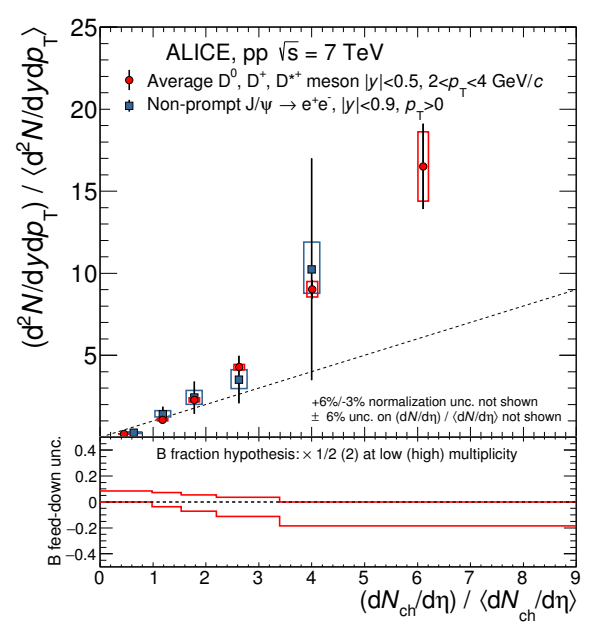

(c) D meson with $2<p_{\mathrm{T}}<4 \mathrm{GeV} / c$ and non-prompt $\mathrm{J} / \psi$.

Figure 8. Average $\mathrm{D}$ meson and $\mathrm{J} / \psi$ relative yields as a function of the relative charged-particle multiplicity at central rapidity. D-meson yields are shown for $2<p_{\mathrm{T}}<4 \mathrm{GeV} / c$, while $\mathrm{J} / \psi$ yields are for $p_{\mathrm{T}}>0$. (a) Inclusive $\mathrm{J} / \psi$ results for $|y|<0.9$ are represented by empty black circles [40], inclusive $\mathrm{J} / \psi$ results for $2.5<y<4.0$ by black filled symbols [40], and prompt $\mathrm{D}$ mesons by red filled circles. (b) Prompt $\mathrm{J} / \psi$ results for $|y|<0.9$ are represented by green filled crosses, and prompt D mesons by red filled circles. (c) Non-prompt $\mathrm{J} / \psi$ results for $|y|<0.9$ are represented by blue filled squares, and prompt D mesons by red filled circles. The relative yields are presented on the top panels with their statistical (vertical bars) and systematic (boxes) uncertainties except the uncertainty on the feed-down fraction for D mesons, which is drawn separately on the bottom panels. The points are located on the x-axis at the average value of $\left(\mathrm{d} N_{\mathrm{ch}} / \mathrm{d} \eta\right) /\left\langle\mathrm{d} N_{\mathrm{ch}} / \mathrm{d} \eta\right\rangle$. The diagonal (dashed) line is drawn to guide the eye. 
energies [27-29]. It could also be explained by the naive picture that processes with large momentum exchange might be associated to a larger amount of gluon-radiation at LHC energies, but no specific model implementation of this effect exists yet. The comparison of the results with model calculations is shown in the next section.

\section{Comparison to theoretical calculations}

Figures 4, 7 and 8 evidence a correlation between heavy-flavour and charged-particle multiplicities. Heavy-flavour production is dominated by hard processes, while charged-particle yields are associated to the soft momentum scale processes. It is then interesting to compare our results with calculations of event generators, designed to be as close as possible to real events in their description of the hard and soft components. Even though several event generators are available, few of them include heavy quarks in a consistent way. One of these is PYTHIA [30, 31], which will be discussed in more detail in section 7.1. In section 7.2 a comparison to PYTHIA 8 [31], to the EPOS 3 [71, 72] event generator results and to a percolation model $[41,73]$ calculation are presented.

\subsection{PYTHIA 8 simulations}

PYTHIA 8 [31] is the $\mathrm{C}++$ successor of PYTHIA 6 [30]. One of the major improvements in PYTHIA 8 with respect to PYTHIA 6 concerns the treatment of the MPI scenario, where the $\mathrm{c}$ and b quarks can be involved in MPI $2 \rightarrow 2$ hard subprocesses. This model improvement is fundamental for an understanding of heavy-flavour production as a function of multiplicity, as MPI can contribute to the observed phenomena. Here PYTHIA 8.157 simulations with the "SoftQCD" process selection including colour reconnection and diffractive processes $^{3}$ are discussed, which will be referred to as PYTHIA 8.

Heavy-flavour production in PYTHIA 8 proceeds via four main mechanisms: (i) The first (hardest) hard process, where the initial c/b quarks originate from the first $2 \rightarrow 2$ hard process, mostly by gluon fusion $(g g \rightarrow c \bar{c})$ or involving a $\mathrm{c} / \mathrm{b}$ sea-quark (e.g. $\mathrm{cu} \rightarrow \mathrm{cu}$ ). (ii) The subsequent hard processes in MPI, produced via the same mechanisms as the first hard process but in consecutive interactions, that we refer to as hard process in MPI. Each produced gluon has a probability to split into a $c \bar{c}$ or b $\bar{b}$ pair contributing to heavy-flavour production. When the initial gluon originates from a hard process, either the first one or a subsequent process (in MPI), we refer to this process as (iii) gluon splitting from hard process. When the initial gluon originates from initial or final state radiation, we refer to this process as (iv) ISR/FSR.

The contribution of the various production processes to the total D- and B-meson production in PYTHIA 8 for pp collisions at $\sqrt{s}=7 \mathrm{TeV}$ is summarised in table 2 . In the following, $\mathrm{D}$ mesons refer to the average of $\mathrm{D}^{0}, \mathrm{D}^{+}$, and $\mathrm{D}^{*+}$, while $\mathrm{B}$ mesons represent the average of $\mathrm{B}^{0}, \mathrm{~B}^{+}$, and $\mathrm{B}^{*+}$. Initial and final state radiation are the main contributors to open heavy-flavour production in PYTHIA 8, corresponding to $\sim 62 \%$ for D mesons and $\sim 40 \%$ for B mesons. MPI correspond to $\sim 21 \%(\sim 24 \%)$ of the D-meson (B-meson)

\footnotetext{
${ }^{3}$ In this simulation, single- and double-diffractive processes contribute to about $20 \%$ of the cross section.
} 


\begin{tabular}{|c|c|c|c|}
\hline Origin of $\mathrm{c}$ and $\mathrm{b}$ quark content & D mesons & B mesons & \\
\hline First hard process & $11 \%$ & $36 \%$ & \\
\hline gluon fusion & $2 \%$ & & $15 \%$ \\
\hline c/b sea & $9 \%$ & & $21 \%$ \\
\hline Hard process in MPI & $21 \%$ & $24 \%$ & \\
\hline Gluon splitting from hard process & $6 \%$ & included in ISR/FSR & \\
\hline ISR/FSR & $62 \%$ & $40 \%$ & \\
\hline Remnant & $<0.2 \%$ & $<0.4 \%$ & \\
\hline
\end{tabular}

Table 2. Contribution of the different production processes to the total D- and B-meson production in PYTHIA 8.157 [31] for pp collisions at $\sqrt{s}=7 \mathrm{TeV}$.

production, while the first hard process is contributing $\sim 11 \%$ for $\mathrm{D}$ mesons and $\sim 36 \%$ for $\mathrm{B}$ mesons. It should be noted that in PYTHIA 8 the largest contribution to hard processes comes from c sea-quarks and not from gluon fusion [74].

Figure 9 (top panels) shows the D and B-meson production as a function of the relative charged-particle multiplicity calculated with PYTHIA 8. The distributions for the main production processes are shown independently. The top-left panel presents results for D mesons, revealing an increasing trend of the relative yields as a function of the relative charged-particle multiplicity for MPI, the gluon splitting from hard processes, and the ISR/FSR contributions. This is consistent with the fact that in PYTHIA 8 MPI and ISR/FSR contribute both to the total multiplicity and to heavy-flavour production. The first hard process contribution instead shows a weaker dependence on the multiplicity: a slight increase is observed at low multiplicities $\left(\mathrm{d} N_{\mathrm{ch}} / \mathrm{d} \eta /\left\langle\mathrm{d} N_{\mathrm{ch}} / \mathrm{d} \eta\right\rangle<1\right)$ followed by a saturation. The picture for B mesons, on the top-right panel, presents similar features as that of $\mathrm{D}$ mesons. The trend for the first hard process contribution shows an increase at low multiplicities and then saturates. The relative charged-particle multiplicity at which the plateau sets in is higher for B than for D mesons. The other contributions to particle production increase faster with multiplicity for B than for D mesons. These differences can be understood as being due to the larger B-meson mass, allowing a larger event activity in MPI and ISR/FSR processes.

Figure 9 (bottom panels) presents the D-meson relative yields as a function of the relative charged-particle multiplicity in PYTHIA 8 for five $p_{\mathrm{T}}$ intervals. The bottom-left panel shows the trend for the sum of all contributions, where an overall linear behaviour is observed, the slope of which increases with $p_{\mathrm{T}}$. The bottom-right panel shows the $p_{\mathrm{T}}$ evolution for the first hard processes only. The relative D-meson yield decreases with multiplicity at low $p_{\mathrm{T}}\left(1<p_{\mathrm{T}}<2 \mathrm{GeV} / c\right)$, while at high $p_{\mathrm{T}}\left(12<p_{\mathrm{T}}<20 \mathrm{GeV} / c\right)$ it exhibits a linear increase. This feature is caused in PYTHIA 8 by the fact that MPI are ordered by their hardness, i.e. the $p_{\mathrm{T}}$ of the first hard scattering is an upper limit for the subsequent hard scatterings and the related ISR/FSR. Thus, charm and beauty production at low $p_{\mathrm{T}}$ is associated mostly with low multiplicity events, whereas heavyflavour hadron production in high $p_{\mathrm{T}}$ intervals is associated to higher multiplicity events. For completeness, the contribution of MPI to the total charged-particle multiplicity was 


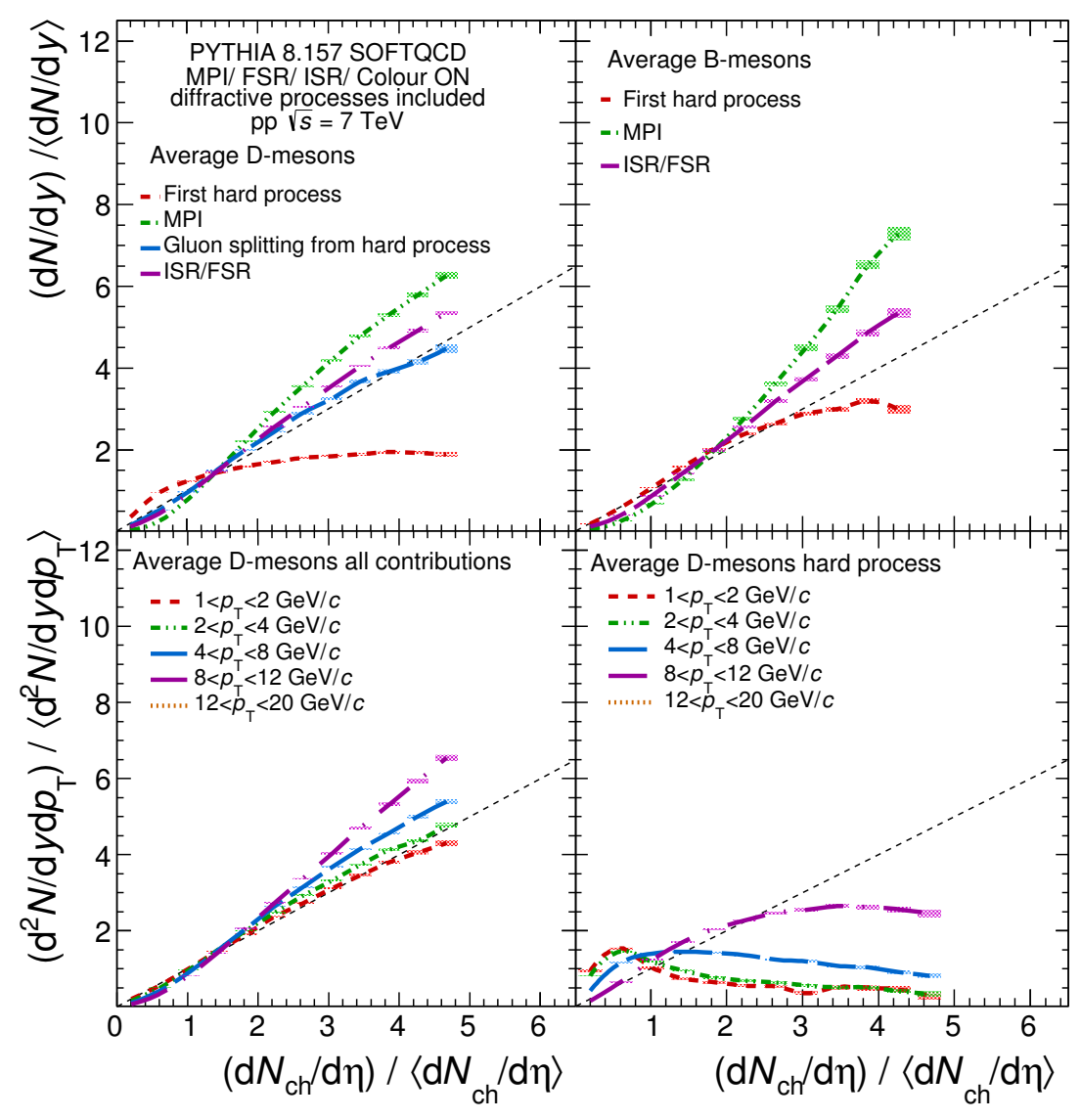

Figure 9. D- and B-meson relative yield as a function of the relative charged-particle multiplicity at central rapidity calculated with the PYTHIA 8.157 event generator [31]. The different $c$ and b quark production processes are separated on the top panels: first hard process, hard process in multiple interactions (MPI), gluon splitting from hard processes and initial/final state radiation (ISR/FSR). The bottom panels present the multiplicity dependence in several $p_{\mathrm{T}}$ intervals for prompt D-meson production, on the left for all contributions and on the right for first hard process only. The coloured lines represent the calculation distributions, whereas the shaded bands represent their statistical uncertainties at given values of $\left(\mathrm{d} N_{\mathrm{ch}} / \mathrm{d} \eta\right) /\left\langle\mathrm{d} N_{\mathrm{ch}} / \mathrm{d} \eta\right\rangle$. The diagonal (dashed) line is drawn to guide the eye.

studied. Only events with a small number of MPI contribute to the low multiplicity intervals, while high multiplicity events are dominated by a large number of MPI, e.g. events with about five times the average multiplicity can have more than 16 parton-parton interactions in the event.

In the following, the multiplicity dependence for D and B-meson production including all contributions in a given $p_{\mathrm{T}}$ interval, as shown in figure 9 (bottom-left panel) for Dmesons, is compared to the measurements.

\subsection{Comparison of data with models}

Figure 10 shows the comparison between D-meson (average of $\mathrm{D}^{0}, \mathrm{D}^{+}$and $\mathrm{D}^{*+}$ ) production and theoretical calculations in four $p_{\mathrm{T}}$ intervals. The results of the PYTHIA $8[30,31]$ 


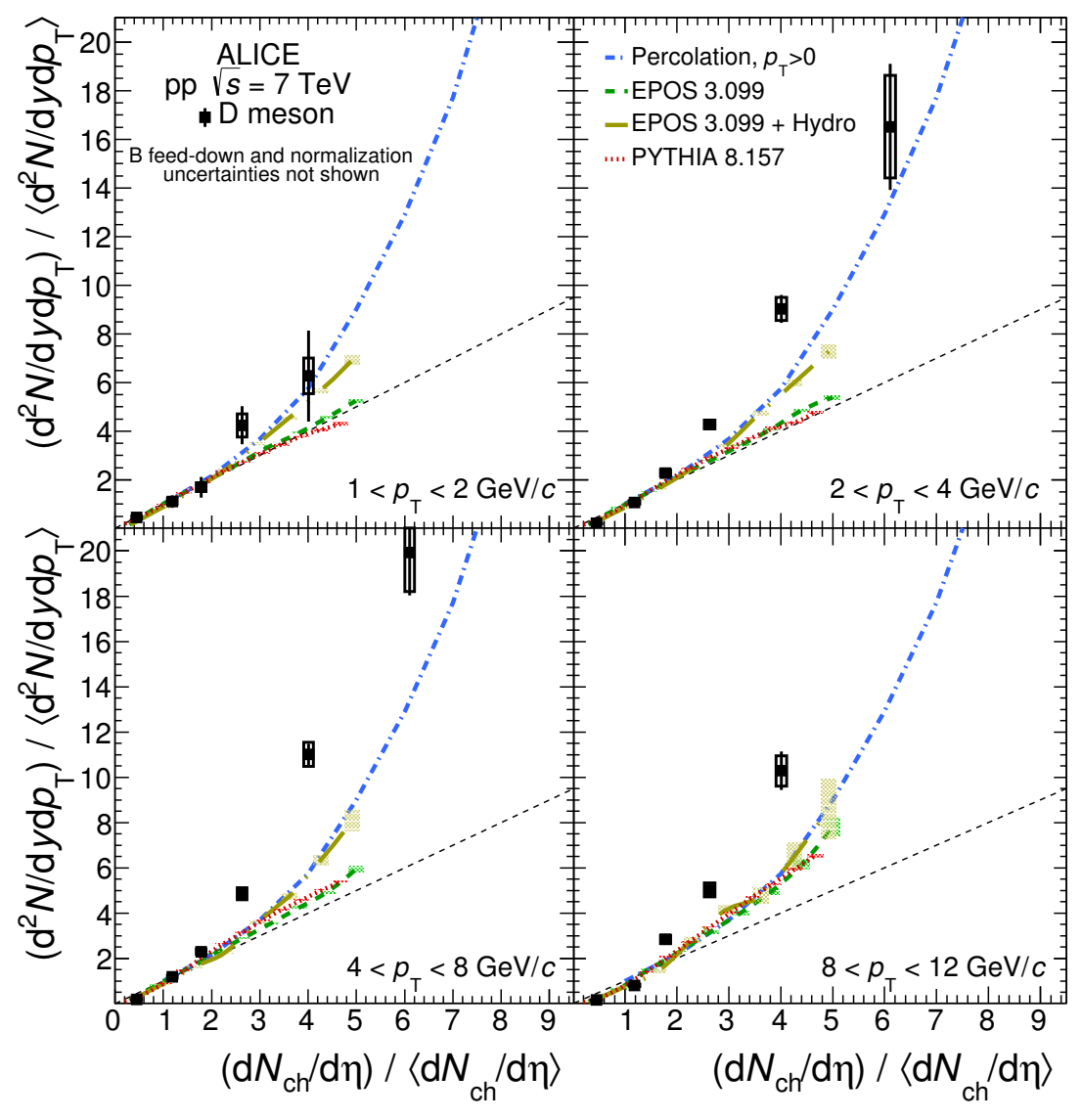

Figure 10. Average D-meson relative yield as a function of the relative charged-particle multiplicity at central rapidity in different $p_{\mathrm{T}}$ intervals. The systematic uncertainties on the data normalisation $(+6 \% /-3 \%)$, on the $\left(\mathrm{d} N_{\mathrm{ch}} / \mathrm{d} \eta\right) /\left\langle\mathrm{d} N_{\mathrm{ch}} / \mathrm{d} \eta\right\rangle$ values $( \pm 6 \%)$, and on the feed down contribution are not shown in this figure. Different calculations are presented: PYTHIA 8.157 [30, 31], EPOS 3 with and without hydro $[71,72]$ and a $p_{\mathrm{T}}$-integrated percolation model $[41,73]$. The coloured lines represent the calculation curves, whereas the shaded bands represent their statistical uncertainties at given values of $\left(\mathrm{d} N_{\mathrm{ch}} / \mathrm{d} \eta\right) /\left\langle\mathrm{d} N_{\mathrm{ch}} / \mathrm{d} \eta\right\rangle$. The diagonal (dashed) line is shown to guide the eye.

and the EPOS 3 [71, 72] event generators, and of percolation calculations [41, 73] are represented by the red dotted line, green dashed or long-dashed and dotted line, and the blue dot-dashed line, respectively. The description of the PYTHIA 8 setup was discussed in section 7.1. Figure 11 presents $p_{\mathrm{T}}$-integrated non-prompt $\mathrm{J} / \psi$ results together with PYTHIA $8[30,31]$ calculations. The percolation model assumes that high-energy hadronic collisions are driven by the exchange of colour sources between the projectile and target in the collision [41, 73]. These colour sources have a finite spatial extension and can interact. In a high-density environment, the coherence among the sources leads to a reduction of their effective number. The source transverse mass determines its transverse size $\left(\propto 1 / m_{\mathrm{T}}\right)$, and allows to distinguish between soft (light) and hard (heavy) sources. As a consequence, at high densities the total charged-particle multiplicity, which originates from soft sources, is reduced. In contrast, hard particle production is less affected due to the smaller transverse 


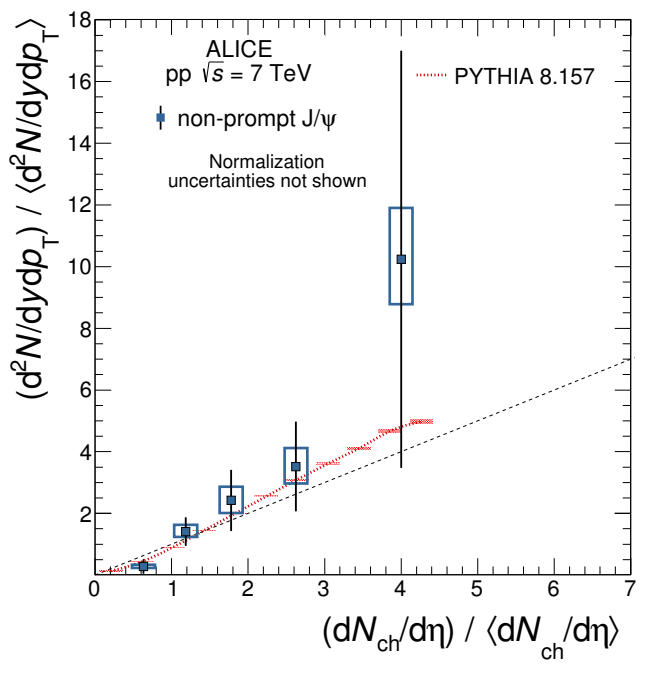

Figure 11. Non-prompt $\mathrm{J} / \psi$ relative yield as a function of the relative charged-particle multiplicity at central rapidity for $p_{\mathrm{T}}>0$. The systematic uncertainties on the data normalisation $(+6 \% /-3 \%)$ and on the $\left(\mathrm{d} N_{\mathrm{ch}} / \mathrm{d} \eta\right) /\left\langle\mathrm{d} N_{\mathrm{ch}} / \mathrm{d} \eta\right\rangle$ values $( \pm 6 \%)$ are not shown in this figure. PYTHIA 8.157 [31] calculation for B mesons is also presented. The coloured line represents the calculation curve, whereas the shaded band represents its uncertainty at given values of $\left(\mathrm{d} N_{\mathrm{ch}} / \mathrm{d} \eta\right) /\left\langle\mathrm{d} N_{\mathrm{ch}} / \mathrm{d} \eta\right\rangle$. The diagonal (dashed) line is shown to guide the eye.

size of hard sources. The percolation model predicts a faster-than-linear increase of heavy flavour relative production with the relative charged-particle multiplicity. The D-meson $p_{\mathrm{T}}$-integrated percolation calculation is represented in all panels of figure 10, even though in this scenario a $p_{\mathrm{T}}$ dependence of the results is expected, such that the higher the $p_{\mathrm{T}}$ of the particle the stronger the deviation from the linear expectation.

EPOS $3[71,72]$ is an event generator for various colliding systems: pp, p-A and $\mathrm{A}-\mathrm{A}$. This event generator imposes the same theoretical scheme in all the systems, i.e. it assumes initial conditions followed by a hydrodynamical evolution. Initial conditions are generated in the Gribov-Regge multiple scattering framework, using the "Parton based Gribov-Regge" formalism [71]. Individual scatterings are referred to as Pomerons, and are identified with parton ladders. Each parton ladder is composed of a pQCD hard process with initial and final state radiation. Non-linear effects are considered by means of a saturation scale. The hadronisation is performed with a string fragmentation procedure. Based on these initial conditions, a hydrodynamical evolution can be applied on the dense core of the collision (3+1D viscous hydrodynamics) [72]. An evaluation within the EPOS 3 model shows that the energy density reached in pp collisions at $\sqrt{s}=7 \mathrm{TeV}$ is high enough to apply such hydrodynamic evolution [72]. Here we discuss the results of an EPOS 3.099 calculation without jet-bulk interaction, which is a process that produces hadrons from hard partons and quarks from the fluid. EPOS 3 without the hydro component (green dashed line in figure 10) predicts an approximately linear increase of D-meson production as a function of the charged-particle multiplicity. This linear scaling shows a $p_{\mathrm{T}}$ dependence, as observed in PYTHIA 8 with the colour reconnection scenario (red dotted line in figure 10), 
although the results differ in magnitude. In EPOS 3, a consequence of the Parton based Gribov-Regge approach is that the number of MPIs is directly related to the multiplicity, i.e. $N_{\text {hard process }} \propto N_{\text {ch }} \propto N_{\text {MPI }}$. When the hydrodynamic evolution is considered (green longdashed and dotted line in figure 10), one observes a departure from a linear multiplicity dependence which is qualitatively comparable to that of the $p_{\mathrm{T}}$-integrated percolation calculation.

The measurements, see figure 10 and figure 11, provide evidence for an increase of the relative heavy-flavour yields with the relative charged-particle multiplicity which proceeds faster than linearly for high multiplicities. This result tends to favour calculations with a substantial deviation from linearity at high multiplicities such as EPOS 3.099 with hydrodynamics or the percolation model.

\section{Summary}

Charm and beauty hadron production as a function of the charged-particle multiplicity was studied in pp collisions at $\sqrt{s}=7 \mathrm{TeV}$. Charged-particle multiplicity at central rapidity was evaluated for events with at least a charged particle in the interval $|\eta|<1$.0. Prompt $\mathrm{D}^{0}$, $\mathrm{D}^{+}$and $\mathrm{D}^{*+}$ meson yields were measured at central rapidity $(|y|<0.5)$ in their hadronic decay channels in five $p_{\mathrm{T}}$ intervals, from $1 \mathrm{GeV} / c$ to $20 \mathrm{GeV} / c$. The increase of the relative yield with increasing charged-particle multiplicity was found to be similar for Dmeson species in all investigated $p_{\mathrm{T}}$ intervals. The average of the $\mathrm{D}^{0}, \mathrm{D}^{+}$and $\mathrm{D}^{*+}$ relative yields increase with the relative charged-particle multiplicity faster than linearly at high multiplicities. No $p_{\mathrm{T}}$ dependence is observed within the current statistical and systematic uncertainties. The lack of quantitative model estimates of the $p_{\mathrm{T}}$ dependence together with the measurement uncertainties prevent to conclude on a possible $p_{\mathrm{T}}$ dependence. A relative yield enhancement of about a factor of 15 with respect to the multiplicity integrated value is observed for events with six times the average charged-particle multiplicity. Prompt $\mathrm{D}^{0}$ relative yields were also measured as a function of the relative charged-particle multiplicity determined in the pseudo-rapidity intervals $-3.7<\eta<-1.7$ and $2.8<\eta<5$. . The results were found to be consistent with those obtained using the charged-particle multiplicity measured at central rapidity. $\mathrm{J} / \psi$ inclusive yields were measured earlier at central rapidity $(|y|<0.9)$ in their di-electron decay channel [40]. The non-prompt $\mathrm{J} / \psi$ contribution was evaluated for $p_{\mathrm{T}}>1.3 \mathrm{GeV} / c$, and extrapolated to $p_{\mathrm{T}}>0$. The non-prompt $\mathrm{J} / \psi$ fraction does not show a dependence on the charged-particle multiplicity at central rapidity.

Open charm, open beauty, and hidden charm hadron yields exhibit a similar increase with the charged-particle multiplicity at central rapidity. This suggests that heavy-flavour relative yields enhancement is not significantly influenced by hadronisation, but more likely directly related to the $c \bar{c}$ and $b \bar{b}$ production processes. The heavy-flavour relative yield enhancement as a function of the charged-particle multiplicity is qualitatively described by: (a) PYTHIA 8.157 calculations including the MPI contributions to particle production [31], (b) percolation model estimates of the influence of colour charge exchanges during the interaction $[41,73]$, (c) predictions by the EPOS 3 event generator which provides a description of the initial conditions followed by a hydrodynamical evolution [71, 72]. How- 
ever, the PYTHIA 8.157 [31] event generator seems to under-estimate the increase of heavy flavour yields with the charged-particle multiplicity at high multiplicities.

\section{Acknowledgments}

We would like to thank P. Skands, co-author of PYTHIA 8, and K. Werner and B. Guiot, coauthors of EPOS 3, for fruitful discussions and for providing their theoretical calculations.

The ALICE Collaboration would like to thank all its engineers and technicians for their invaluable contributions to the construction of the experiment and the CERN accelerator teams for the outstanding performance of the LHC complex. The ALICE Collaboration gratefully acknowledges the resources and support provided by all Grid centres and the Worldwide LHC Computing Grid (WLCG) collaboration. The ALICE Collaboration acknowledges the following funding agencies for their support in building and running the ALICE detector: State Committee of Science, World Federation of Scientists (WFS) and Swiss Fonds Kidagan, Armenia, Conselho Nacional de Desenvolvimento Científico e Tecnológico $(\mathrm{CNPq})$, Financiadora de Estudos e Projetos (FINEP), Fundação de Amparo à Pesquisa do Estado de São Paulo (FAPESP); National Natural Science Foundation of China (NSFC), the Chinese Ministry of Education (CMOE) and the Ministry of Science and Technology of China (MSTC); Ministry of Education and Youth of the Czech Republic; Danish Natural Science Research Council, the Carlsberg Foundation and the Danish National Research Foundation; The European Research Council under the European Community's Seventh Framework Programme; Helsinki Institute of Physics and the Academy of Finland; French CNRS-IN2P3, the 'Region Pays de Loire', 'Region Alsace', 'Region Auvergne' and CEA, France; German Bundesministerium fur Bildung, Wissenschaft, Forschung und Technologie (BMBF) and the Helmholtz Association; General Secretariat for Research and Technology, Ministry of Development, Greece; Hungarian Orszagos Tudomanyos Kutatasi Alappgrammok (OTKA) and National Office for Research and Technology (NKTH); Department of Atomic Energy and Department of Science and Technology of the Government of India; Istituto Nazionale di Fisica Nucleare (INFN) and Centro Fermi - Museo Storico della Fisica e Centro Studi e Ricerche "Enrico Fermi", Italy; MEXT Grant-in-Aid for Specially Promoted Research, Japan; Joint Institute for Nuclear Research, Dubna; National Research Foundation of Korea (NRF); Consejo Nacional de Cienca y Tecnologia (CONACYT), Direccion General de Asuntos del Personal Academico(DGAPA), México, Amerique Latine Formation academique - European Commission (ALFA-EC) and the EPLANET Program (European Particle Physics Latin American Network); Stichting voor Fundamenteel Onderzoek der Materie (FOM) and the Nederlandse Organisatie voor Wetenschappelijk Onderzoek (NWO), Netherlands; Research Council of Norway (NFR); National Science Centre, Poland; Ministry of National Education/Institute for Atomic Physics and National Council of Scientific Research in Higher Education (CNCSI-UEFISCDI), Romania; Ministry of Education and Science of Russian Federation, Russian Academy of Sciences, Russian Federal Agency of Atomic Energy, Russian Federal Agency for Science and Innovations and The Russian Foundation for Basic Research; Ministry of Education of Slovakia; Department of Science and Technology, South Africa; Centro de Investiga- 
ciones Energeticas, Medioambientales y Tecnologicas (CIEMAT), E-Infrastructure shared between Europe and Latin America (EELA), Ministerio de Economía y Competitividad (MINECO) of Spain, Xunta de Galicia (Consellería de Educación), Centro de Aplicaciones Tecnológicas y Desarrollo Nuclear (CEADEN), Cubaenergía, Cuba, and IAEA (International Atomic Energy Agency); Swedish Research Council (VR) and Knut \& Alice Wallenberg Foundation (KAW); Ukraine Ministry of Education and Science; United Kingdom Science and Technology Facilities Council (STFC); The United States Department of Energy, the United States National Science Foundation, the State of Texas, and the State of Ohio; Ministry of Science, Education and Sports of Croatia and Unity through Knowledge Fund, Croatia. Council of Scientific and Industrial Research (CSIR), New Delhi, India.

\section{A Tables of the results}

Table 4 reports the results of the relative average D-meson yields per inelastic collision as a function of the relative charged-particle multiplicity at mid-rapidity in several D-meson transverse momentum intervals, see figure 4 . The corresponding relative average D-meson yields normalised to the visible cross section instead of the inelastic one are presented in table 3.

Table 6 summarises the relative $\mathrm{D}^{0}$ yields per inelastic collision as a function of the relative raw multiplicity measured with the V0 detector at forward rapidity, see figure 5. These relative $\mathrm{D}^{0}$ yields are also presented in table 5 normalised to the visible cross section.

Table 7 reports the fraction of non-prompt $\mathrm{J} / \psi$ to the inclusive $\mathrm{J} / \psi$ yields as a function of the relative charged-particle multiplicity at mid-rapidity, see figure 7 . The relative prompt and non-prompt $\mathrm{J} / \psi$ yields per inelastic collision are reported in table 8 , while table 7 presents these yields normalised to the visible cross section. 


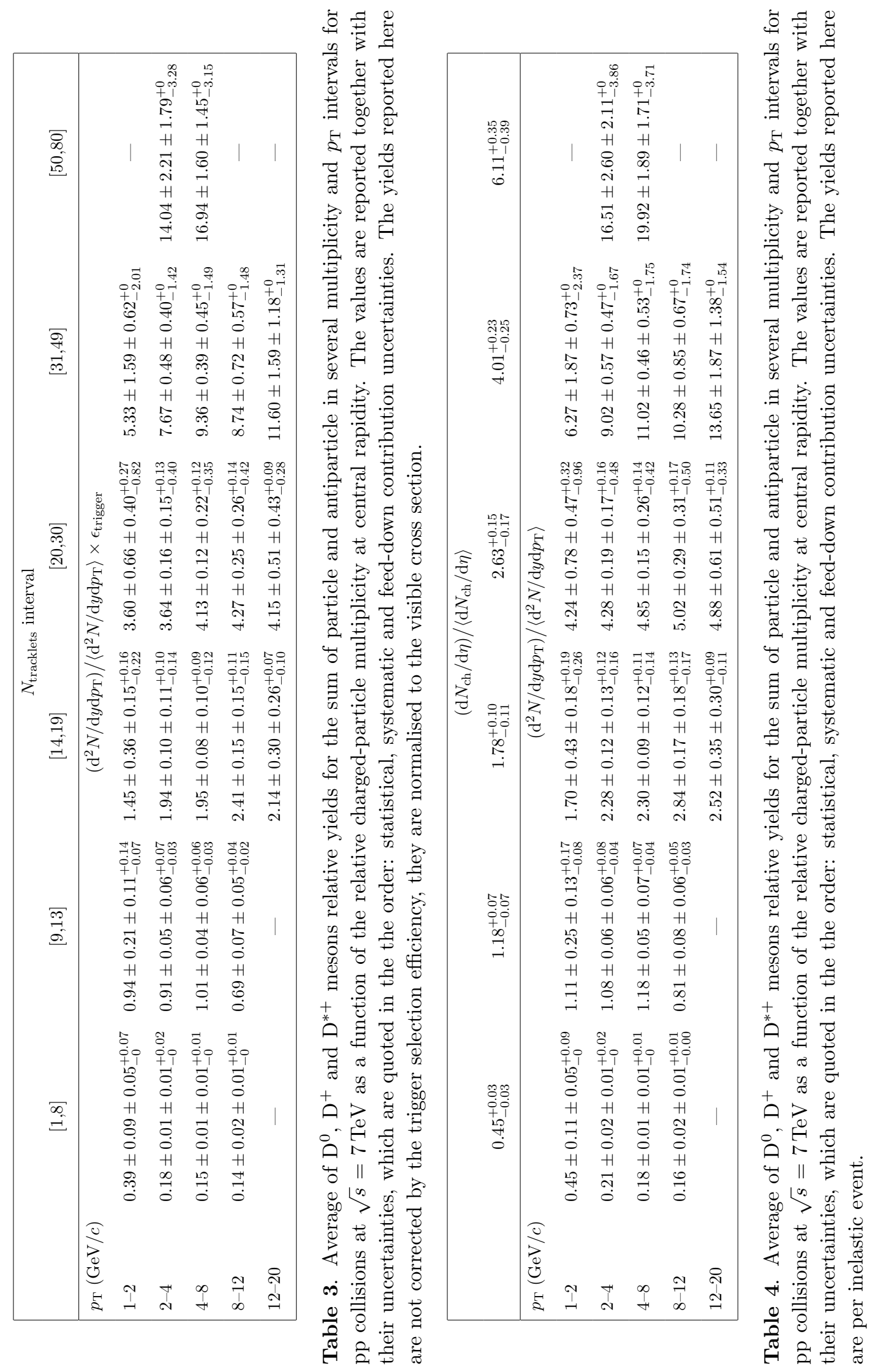



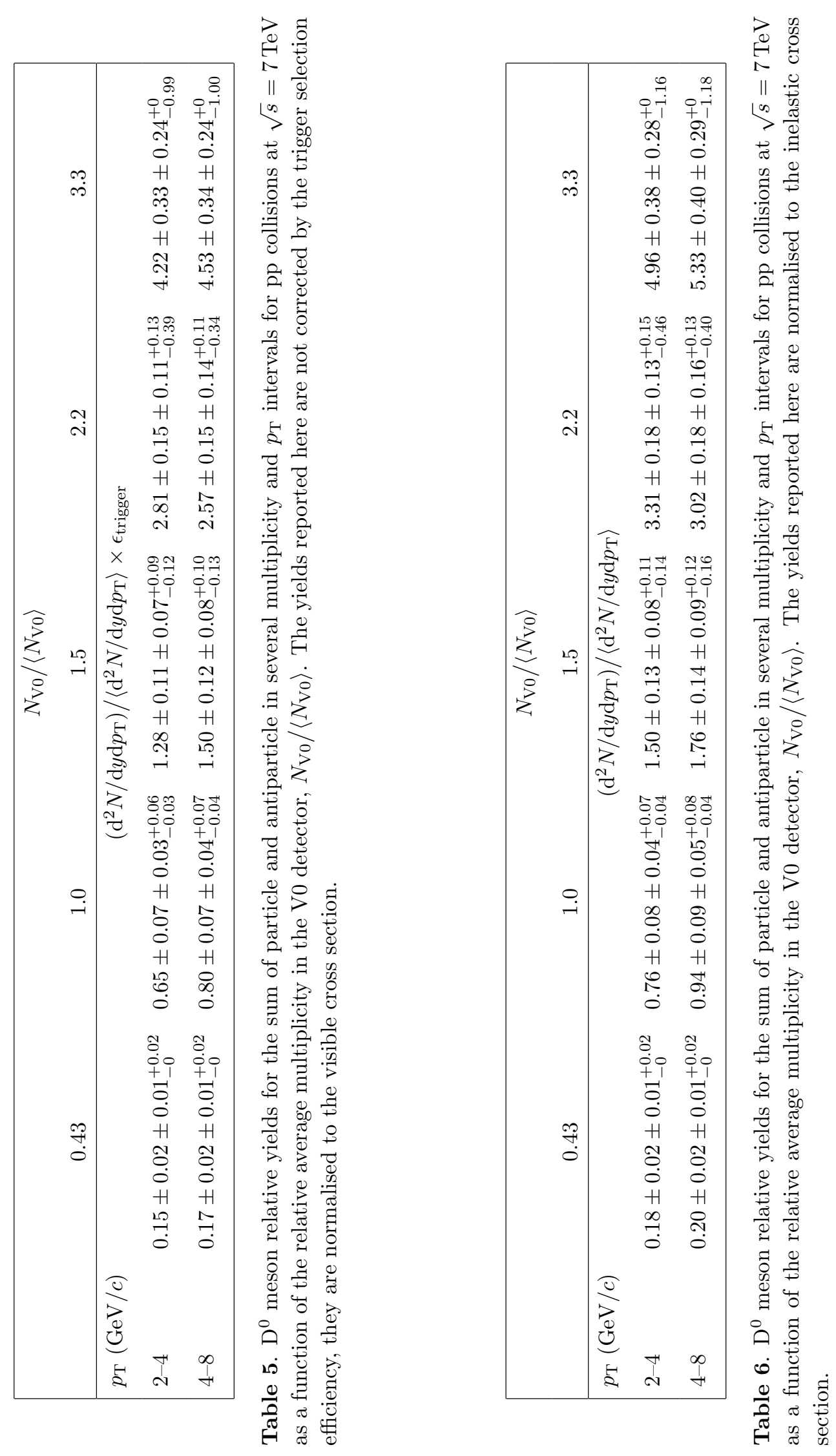


\begin{tabular}{|ccccc}
\hline$N_{\text {tracklets }}$ & $f_{\mathrm{B}}(\%)$ & $f_{\mathrm{B}}^{\text {extr }}(\%)$ & $\left(\mathrm{d} N_{\mathrm{J} / \psi}^{\text {prompt }} / \mathrm{d} y\right) /\left\langle\mathrm{d} N_{\mathrm{J} / \psi}^{\text {prompt }} / \mathrm{d} y\right\rangle \times \epsilon_{\text {trigger }}$ & $\left(\mathrm{d} N_{\mathrm{J} / \psi}^{\text {non }- \text { prompt }} / \mathrm{d} y\right) /\left\langle\mathrm{d} N_{\mathrm{J} / \psi}^{\text {non }- \text { prompt }} / \mathrm{d} y\right\rangle \times \epsilon_{\text {trigger }}$ \\
\hline$[4,8]$ & $10.1 \pm 7.8 \pm 2.5$ & $10.2 \pm 7.9 \pm 2.5$ & $0.37 \pm 0.07 \pm 0.01$ & $0.24 \pm 0.20_{-0.04}^{+0.05}$ \\
{$[9,13]$} & $20.8 \pm 6.9 \pm 2.7$ & $20.9 \pm 6.9 \pm 2.7$ & $0.80 \pm 0.14 \pm 0.04$ & $1.20 \pm 0.39_{-0.14}^{+0.19}$ \\
{$[14,19]$} & $15.6 \pm 6.3 \pm 2.0$ & $15.7 \pm 6.3 \pm 2.0$ & $1.95 \pm 0.31 \pm 0.24$ & $2.06 \pm 0.84_{-0.35}^{+0.37}$ \\
{$[20,30]$} & $16.7 \pm 6.7 \pm 3.3$ & $16.8 \pm 6.7 \pm 3.3$ & $2.61 \pm 0.46 \pm 0.27$ & $2.99 \pm 1.23_{-0.47}^{+0.51}$ \\
{$[31,49]$} & $19.0 \pm 11.9 \pm 4.2$ & $19.0 \pm 12.0 \pm 4.2$ & $6.50 \pm 1.50 \pm 0.31$ & $8.70 \pm 5.75_{-1.24}^{+1.41}$ \\
\hline
\end{tabular}

Table 7. Fraction of non-prompt $\mathrm{J} / \psi$ measured for $p_{\mathrm{T}}>1.3 \mathrm{GeV} / c, f_{\mathrm{B}}(\%)$, and extrapolated down to $p_{\mathrm{T}}>0, f_{\mathrm{B}}^{\text {extr }}(\%)$ in the various $N_{\text {tracklets }}$ intervals. Prompt and non-prompt $\mathrm{J} / \psi$ relative yields for $p_{\mathrm{T}}>0$ are also reported in the different multiplicity intervals. The first and second uncertainties correspond to the statistical and systematic uncertainties, respectively. The yields reported here are not corrected by the trigger selection efficiency, they are normalised to the visible cross section.

\begin{tabular}{|ccc|}
\hline$\left(\mathrm{d} N_{\mathrm{ch}} / \mathrm{d} \eta\right) /\left\langle\mathrm{d} N_{\mathrm{ch}} / \mathrm{d} \eta\right\rangle$ & $\left(\mathrm{d} N_{\mathrm{J} / \psi}^{\text {prompt }} / \mathrm{d} y\right) /\left\langle\mathrm{d} N_{\mathrm{J} / \psi}^{\text {prompt }} / \mathrm{d} y\right\rangle$ & $\left(\mathrm{d} N_{\mathrm{J} / \psi}^{\text {non-prompt }} / \mathrm{d} y\right) /\left\langle\mathrm{d} N_{\mathrm{J} / \psi}^{\text {non-prompt }} / \mathrm{d} y\right\rangle$ \\
\hline $0.63_{-0.4}^{+0.4}$ & $0.44 \pm 0.08 \pm 0.01$ & $0.28 \pm 0.23_{-0.05}^{+0.06}$ \\
$1.18_{-0.07}^{+0.07}$ & $0.94 \pm 0.17 \pm 0.05$ & $1.41 \pm 0.46_{-0.17}^{+0.22}$ \\
$1.78_{-0.11}^{+0.10}$ & $2.29 \pm 0.36 \pm 0.28$ & $2.42 \pm 0.99_{-0.41}^{+0.44}$ \\
$2.63_{-0.17}^{+0.15}$ & $3.07 \pm 0.54 \pm 0.32$ & $3.52 \pm 1.45_{-0.55}^{+0.60}$ \\
$4.01_{-0.25}^{+0.23}$ & $7.65 \pm 1.76 \pm 0.36$ & $10.24 \pm 6.76_{-1.46}^{+1.66}$ \\
\hline
\end{tabular}

Table 8. Prompt and non-prompt $\mathrm{J} / \psi$ relative yields for $p_{\mathrm{T}}>0$ in different multiplicity bins. The first and second uncertainties correspond to the statistical and systematic uncertainties respectively. The yields reported here are normalised to the inelastic cross section.

Open Access. This article is distributed under the terms of the Creative Commons Attribution License (CC-BY 4.0), which permits any use, distribution and reproduction in any medium, provided the original author(s) and source are credited.

\section{References}

[1] ALICE collaboration, Measurement of charm production at central rapidity in proton-proton collisions at $\sqrt{s}=7 \mathrm{TeV}$, JHEP 01 (2012) 128 [arXiv:1111.1553] [INSPIRE].

[2] ALICE collaboration, Measurement of charm production at central rapidity in proton-proton collisions at $\sqrt{s}=2.76 \mathrm{TeV}$, JHEP 07 (2012) 191 [arXiv: 1205.4007] [INSPIRE].

[3] LHCb collaboration, Prompt charm production in pp collisions at $\sqrt{s}=7 \mathrm{TeV}$, Nucl. Phys. B 871 (2013) 1 [arXiv:1302.2864] [INSPIRE].

[4] B.A. Kniehl, G. Kramer, I. Schienbein and H. Spiesberger, Inclusive Charmed-Meson Production at the CERN LHC, Eur. Phys. J. C 72 (2012) 2082 [arXiv:1202.0439] [INSPIRE].

[5] M. Cacciari, M. Greco and P. Nason, The $p_{\mathrm{T}}$ spectrum in heavy flavor hadroproduction, JHEP 05 (1998) 007 [hep-ph/9803400] [INSPIRE]. 
[6] M. Cacciari, S. Frixione and P. Nason, The $p_{\mathrm{T}}$ spectrum in heavy flavor photoproduction, JHEP 03 (2001) 006 [hep-ph/0102134] [INSPIRE].

[7] M. Cacciari, S. Frixione, N. Houdeau, M.L. Mangano, P. Nason and G. Ridolfi, Theoretical predictions for charm and bottom production at the LHC, JHEP 10 (2012) 137 [arXiv: 1205.6344] [INSPIRE].

[8] B.A. Kniehl, G. Kramer, I. Schienbein and H. Spiesberger, Reconciling open charm production at the Fermilab Tevatron with QCD, Phys. Rev. Lett. 96 (2006) 012001 [hep-ph/0508129] [INSPIRE].

[9] R. Maciula and A. Szczurek, Open charm production at the LHC: $k_{t}$-factorization approach, Phys. Rev. D 87 (2013) 094022 [arXiv:1301.3033] [INSPIRE].

[10] ALICE collaboration, Measurement of prompt $J / \psi$ and beauty hadron production cross sections at mid-rapidity in pp collisions at $\sqrt{s}=7 \mathrm{TeV}$, JHEP 11 (2012) 065 [arXiv: 1205.5880] [INSPIRE].

[11] ALICE collaboration, Measurement of electrons from beauty hadron decays in pp collisions at $\sqrt{s}=7$ TeV, Phys. Lett. B 721 (2013) 13 [arXiv:1208.1902] [INSPIRE].

[12] ATLAS collaboration, Measurement of the differential cross-section of $B^{+}$meson production in pp collisions at $\sqrt{s}=7 \mathrm{TeV}$ at ATLAS, JHEP 10 (2013) 042 [arXiv:1307.0126] [INSPIRE].

[13] CMS collaboration, Prompt and non-prompt $J / \psi$ production in pp collisions at $\sqrt{s}=7 \mathrm{TeV}$, Eur. Phys. J. C 71 (2011) 1575 [arXiv:1011.4193] [inSPIRE].

[14] LHCb collaboration, Measurement of $\sigma(p p \rightarrow b \bar{b} X)$ at $\sqrt{s}=7 \mathrm{TeV}$ in the forward region, Phys. Lett. B 694 (2010) 209 [arXiv: 1009.2731] [InSPIRE].

[15] B.A. Kniehl, G. Kramer, I. Schienbein and H. Spiesberger, Inclusive B-Meson Production at the LHC in the GM-VFN Scheme, Phys. Rev. D 84 (2011) 094026 [arXiv:1109.2472] [INSPIRE].

[16] CDF collaboration, D. Acosta et al., Measurement of the $J / \psi$ meson and $b$-hadron production cross sections in pp collisions at $\sqrt{s}=1960$ GeV, Phys. Rev. D 71 (2005) 032001 [hep-ex/0412071] [INSPIRE].

[17] M. Cacciari, S. Frixione, M.L. Mangano, P. Nason and G. Ridolfi, $Q C D$ analysis of first $b$ cross-section data at $1.96 \mathrm{TeV}$, JHEP 07 (2004) 033 [hep-ph/0312132] [INSPIRE].

[18] B.A. Kniehl, G. Kramer, I. Schienbein and H. Spiesberger, Finite-mass effects on inclusive B meson hadroproduction, Phys. Rev. D 77 (2008) 014011 [arXiv:0705.4392] [INSPIRE].

[19] L. Frankfurt, M. Strikman and C. Weiss, Dijet production as a centrality trigger for $p p$ collisions at CERN LHC, Phys. Rev. D 69 (2004) 114010 [hep-ph/0311231] [INSPIRE].

[20] L. Frankfurt, M. Strikman and C. Weiss, Transverse nucleon structure and diagnostics of hard parton-parton processes at LHC, Phys. Rev. D 83 (2011) 054012 [arXiv:1009.2559] [INSPIRE].

[21] M.Y. Azarkin, I.M. Dremin and M. Strikman, Jets in multiparticle production in and beyond geometry of proton-proton collisions at the LHC, Phys. Lett. B 735 (2014) 244 [arXiv: 1401.1973] [INSPIRE].

[22] V.N. Gribov, Analytic properties of partial wave amplitudes and asymptotic behaviour of scattering amplitude, Nucl. Phys. 40 (1963) 107. 
[23] M.M. Block and R.N. Cahn, High-Energy pp and pp Forward Elastic Scattering and Total Cross-Sections, Rev. Mod. Phys. 57 (1985) 563 [INSPIRE].

[24] I.M. Dremin and V.A. Nechitailo, Proton periphery activated by multiparticle dynamics, Nucl. Phys. A 916 (2013) 241 [arXiv:1306.5384] [INSPIRE].

[25] M. Strikman, Transverse structure of the nucleon and multiparton interactions, Prog. Theor. Phys. Suppl. 187 (2011) 289 [INSPIRE].

[26] LEBC-EHS collaboration, M. Aguilar-Benitez et al., Comparative Properties of $400 \mathrm{GeV} / \mathrm{c}$ Proton-Proton Interactions With and Without Charm Production, Z. Phys. C 41 (1988) 191 [INSPIRE].

[27] P. Bartalini and L. Fano, 1st International Workshop on Multiple Partonic Interactions at the LHC (MPI@LHC 08), Perugia, Italy, 27-31 October 2008, P. Bartalini and L. Fano eds., DESY, Hamburg (2009) [DESY-PROC-2009-06] [arXiv: 1003.4220] [INSPIRE].

[28] T. Sjöstrand and M. van Zijl, A Multiple Interaction Model for the Event Structure in Hadron Collisions, Phys. Rev. D 36 (1987) 2019 [INSPIRE].

[29] S. Porteboeuf and R. Granier de Cassagnac, J/ $\Psi$ yield vs. multiplicity in proton-proton collisions at the LHC, Nucl. Phys. Proc. Suppl. 214 (2011) 181 [arXiv:1012.0719] [inSPIRE].

[30] T. Sjöstrand, S. Mrenna and P.Z. Skands, PYTHIA 6.4 Physics and Manual, JHEP 05 (2006) 026 [hep-ph/0603175] [INSPIRE].

[31] T. Sjöstrand, S. Mrenna and P.Z. Skands, A Brief Introduction to PYTHIA 8.1, Comput. Phys. Commun. 178 (2008) 852 [arXiv:0710.3820] [INSPIRE].

[32] M. Bahr et al., HERWIG++ Physics and Manual, Eur. Phys. J. C 58 (2008) 639 [arXiv:0803.0883] [INSPIRE].

[33] CMS collaboration, Jet and underlying event properties as a function of charged-particle multiplicity in proton-proton collisions at $\sqrt{s}=7$ TeV, Eur. Phys. J. C 73 (2013) 2674 [arXiv: 1310.4554] [INSPIRE].

[34] ALICE collaboration, Underlying Event measurements in pp collisions at $\sqrt{s}=0.9$ and $7 \mathrm{TeV}$ with the ALICE experiment at the LHC, JHEP 07 (2012) 116 [arXiv:1112.2082] [INSPIRE].

[35] ALICE collaboration, Multiplicity dependence of two-particle azimuthal correlations in $p p$ collisions at the LHC, JHEP 09 (2013) 049 [arXiv: 1307.1249] [INSPIRE].

[36] M. Strikman, Comments on the observation of high multiplicity events at the LHC, Phys. Rev. D 84 (2011) 011501 [arXiv:1105.2285] [INSPIRE].

[37] B.Z. Kopeliovich, H.J. Pirner, I.K. Potashnikova, K. Reygers and I. Schmidt, $J / \psi$ in high-multiplicity pp collisions: Lessons from pA collisions, Phys. Rev. D 88 (2013) 116002 [arXiv: 1308.3638] [INSPIRE].

[38] LHCb collaboration, Observation of double charm production involving open charm in pp collisions at $\sqrt{s}=7 \mathrm{TeV}$, JHEP 06 (2012) 141 [arXiv:1205.0975] [INSPIRE].

[39] LHCb collaboration, Observation of $J / \psi$ pair production in pp collisions at $\sqrt{s}=7 \mathrm{TeV}$, Phys. Lett. B 707 (2012) 52 [arXiv:1109.0963] [INSPIRE].

[40] ALICE collaboration, $J / \psi$ Production as a Function of Charged Particle Multiplicity in pp Collisions at $\sqrt{s}=7 \mathrm{TeV}$, Phys. Lett. B 712 (2012) 165 [arXiv:1202.2816] [INSPIRE]. 
[41] E.G. Ferreiro and C. Pajares, High multiplicity pp events and $J / \psi$ production at LHC, Phys. Rev. C 86 (2012) 034903 [arXiv: 1203.5936] [INSPIRE].

[42] PHOBOS collaboration, B. Alver et al., Phobos results on charged particle multiplicity and pseudorapidity distributions in $A u+A u, C u+C u, d+A u$ and $p+p$ collisions at ultra-relativistic energies, Phys. Rev. C 83 (2011) 024913 [arXiv:1011.1940] [INSPIRE].

[43] ALICE collaboration, Long-range angular correlations of $\pi, K$ and $p$ in $p-P b$ collisions at $\sqrt{s_{\mathrm{NN}}}=5.02 \mathrm{TeV}$, Phys. Lett. B 726 (2013) 164 [arXiv:1307.3237] [INSPIRE].

[44] ALICE collaboration, Long-range angular correlations on the near and away side in $p-P b$ collisions at $\sqrt{s_{\mathrm{NN}}}=5.02 \mathrm{TeV}$, Phys. Lett. B 719 (2013) 29 [arXiv:1212.2001] [inSPIRE].

[45] CMS collaboration, Observation of long-range near-side angular correlations in proton-lead collisions at the LHC, Phys. Lett. B 718 (2013) 795 [arXiv:1210.5482] [INSPIRE].

[46] CMS collaboration, Multiplicity and transverse momentum dependence of two- and four-particle correlations in $p P b$ and PbPb collisions, Phys. Lett. B 724 (2013) 213 [arXiv:1305.0609] [INSPIRE].

[47] ATLAS collaboration, Measurement with the ATLAS detector of multi-particle azimuthal correlations in $p+P b$ collisions at $\sqrt{s_{\mathrm{NN}}}=5.02 \mathrm{TeV}$, Phys. Lett. B 725 (2013) 60 [arXiv: 1303.2084] [INSPIRE].

[48] ATLAS collaboration, Observation of Associated Near-Side and Away-Side Long-Range Correlations in $\sqrt{s_{\mathrm{NN}}}=5.02 \mathrm{TeV}$ Proton-Lead Collisions with the ATLAS Detector, Phys. Rev. Lett. 110 (2013) 182302 [arXiv:1212.5198] [INSPIRE].

[49] CMS collaboration, Observation of Long-Range Near-Side Angular Correlations in Proton-Proton Collisions at the LHC, JHEP 09 (2010) 091 [arXiv: 1009.4122] [InSPIRE].

[50] S. Vogel, P.B. Gossiaux, K. Werner and J. Aichelin, Charm quark energy loss in proton-proton collisions at LHC energies, J. Phys. Conf. Ser. 420 (2013) 012034 [inSPIRE].

[51] ALICE collaboration, The ALICE experiment at the CERN LHC, 2008 JINST 3 S08002 [INSPIRE].

[52] ALICE collaboration, Alignment of the ALICE Inner Tracking System with cosmic-ray tracks, 2010 JINST 5 P03003 [arXiv:1001.0502] [INSPIRE].

[53] ALICE collaboration, The ALICE TPC, a large 3-dimensional tracking device with fast readout for ultra-high multiplicity events, Nucl. Instrum. Meth. A 622 (2010) 316 [arXiv: 1001.1950] [INSPIRE].

[54] ALICE collaboration, Performance of the ALICE Experiment at the CERN LHC, Int. J. Mod. Phys. A 29 (2014) 1430044 [arXiv:1402.4476] [InSPIRE].

[55] ALICE collaboration, Performance of the ALICE VZERO system, 2013 JINST 8 P10016 [arXiv: 1306.3130] [INSPIRE].

[56] ALICE collaboration, Measurement of inelastic, single- and double-diffraction cross sections in proton-proton collisions at the LHC with ALICE, Eur. Phys. J. C 73 (2013) 2456 [arXiv: 1208.4968] [INSPIRE].

[57] ALICE collaboration, Charged-particle multiplicity measurement in proton-proton collisions at $\sqrt{s}=7 \mathrm{TeV}$ with ALICE at LHC, Eur. Phys. J. C 68 (2010) 345 [arXiv:1004.3514] [INSPIRE]. 
[58] Particle Data Group collaboration, K.A. Olive et al., Review of Particle Physics, Chin. Phys. C 38 (2014) 090001 [InSPIRE].

[59] P.Z. Skands, Tuning Monte Carlo Generators: The Perugia Tunes, Phys. Rev. D 82 (2010) 074018 [arXiv: 1005.3457] [INSPIRE].

[60] R. Brun, F. Carminati and S. Giani, GEANT Detector Description and Simulation Tool, CERN-W5013 (1994) [INSPIRE].

[61] D.J. Lange, The EvtGen particle decay simulation package, Nucl. Instrum. Meth. A 462 (2001) 152 [INSPIRE].

[62] H. Fritzsch, Producing Heavy Quark Flavors in Hadronic Collisions: A Test of Quantum Chromodynamics, Phys. Lett. B 67 (1977) 217 [InSPIRE].

[63] J.F. Amundson, O.J.P. Eboli, E.M. Gregores and F. Halzen, Quantitative tests of color evaporation: Charmonium production, Phys. Lett. B 390 (1997) 323 [hep-ph/9605295] [INSPIRE].

[64] P.Z. Skands The Perugia Tunes, FERMILAB-CONF-09-113 [arXiv:0905.3418] [InSPIRE].

[65] ALICE collaboration, Rapidity and transverse momentum dependence of inclusive $J / \psi$ production in pp collisions at $\sqrt{s}=7 \mathrm{TeV}$, Phys. Lett. B 704 (2011) 442 [Erratum ibid. B 718 (2012) 692] [arXiv:1105.0380] [INSPIRE].

[66] F. Bossu, Z.C. del Valle, A. de Falco, M. Gagliardi, S. Grigoryan and G. Martinez Garcia, Phenomenological interpolation of the inclusive J/psi cross section to proton-proton collisions at $2.76 \mathrm{TeV}$ and $5.5 \mathrm{TeV}$, arXiv:1103.2394 [INSPIRE].

[67] ATLAS collaboration, Measurement of the differential cross-sections of inclusive, prompt and non-prompt $J / \psi$ production in proton-proton collisions at $\sqrt{s}=7 \mathrm{TeV}$, Nucl. Phys. B 850 (2011) 387 [arXiv:1104.3038] [INSPIRE].

[68] LHCb collaboration, Measurement of $J / \psi$ production in pp collisions at $\sqrt{s}=7 \mathrm{TeV}$, Eur. Phys. J. C 71 (2011) 1645 [arXiv: 1103. 0423] [INSPIRE].

[69] ALICE collaboration, Neutral pion production at midrapidity in $p p$ and $\mathrm{Pb}-\mathrm{Pb}$ collisions at $\sqrt{s_{\mathrm{NN}}}=2.76 \mathrm{TeV}$, Eur. Phys. J. C 74 (2014) 3108 [arXiv: 1405.3794] [INSPIRE].

[70] ALICE collaboration, Neutral pion and $\eta$ meson production in proton-proton collisions at $\sqrt{s}=0.9 \mathrm{TeV}$ and $\sqrt{s}=7 \mathrm{TeV}$, Phys. Lett. B 717 (2012) 162 [arXiv:1205.5724] [INSPIRE].

[71] H.J. Drescher, M. Hladik, S. Ostapchenko, T. Pierog and K. Werner, Parton based Gribov-Regge theory, Phys. Rept. 350 (2001) 93 [hep-ph/0007198] [InSPIRE].

[72] K. Werner, B. Guiot, I. Karpenko and T. Pierog, Analysing radial flow features in $p-P b$ and $p-p$ collisions at several TeV by studying identified particle production in EPOS3, Phys. Rev. C 89 (2014) 064903 [arXiv: 1312.1233] [INSPIRE].

[73] E.G. Ferreiro and C. Pajares, Open charm production in high multiplicity proton-proton events at the LHC, arXiv:1501.03381 [INSPIRE].

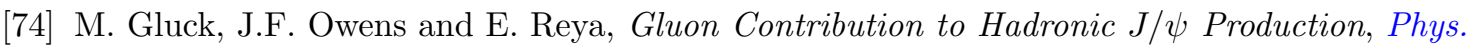
Rev. D 17 (1978) 2324 [INSPIRE]. 


\section{The ALICE collaboration}

J. Adam ${ }^{40}$, D. Adamová ${ }^{83}$, M.M. Aggarwal ${ }^{87}$, G. Aglieri Rinella ${ }^{36}$, M. Agnello ${ }^{111}$,

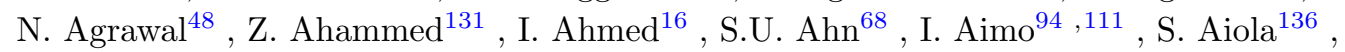
M. Ajaz $^{16}$, A. Akindinov ${ }^{58}$, S.N. Alam ${ }^{131}$, D. Aleksandrov ${ }^{100}$, B. Alessandro ${ }^{111}$, D. Alexandre ${ }^{102}$, R. Alfaro Molina ${ }^{64}$, A. Alici ${ }^{105}, 12$, A. Alkin ${ }^{3}$, J. Alme ${ }^{38}$, T. Alt ${ }^{43}$, S. Altinpinar ${ }^{18}$, I. Altsybeev ${ }^{130}$, C. Alves Garcia Prado ${ }^{119}$, C. Andrei ${ }^{78}$, A. Andronic ${ }^{97}$, V. Anguelov ${ }^{93}$, J. Anielski ${ }^{54}$, T. Antičić ${ }^{98}$, F. Antinori ${ }^{108}$, P. Antonioli ${ }^{105}$, L. Aphecetche ${ }^{113}$ H. Appelshäuser ${ }^{53}$, S. Arcelli ${ }^{28}$, N. Armesto ${ }^{17}$, R. Arnaldi ${ }^{111}$, T. Aronsson ${ }^{136}$, I.C. Arsene ${ }^{22}$,

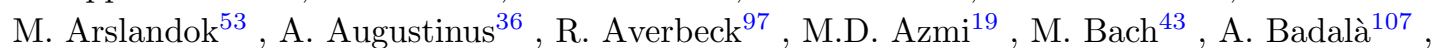
Y.W. Baek ${ }^{44}$, S. Bagnasco ${ }^{111}$, R. Bailhache ${ }^{53}$, R. Bala ${ }^{90}$, A. Baldisseri ${ }^{15}$, M. Ball ${ }^{92}$, F. Baltasar Dos Santos Pedrosa ${ }^{36}$, R.C. Baral ${ }^{61}$, A.M. Barbano ${ }^{111}$, R. Barbera ${ }^{29}$, F. Barile ${ }^{33}$, G.G. Barnaföldi ${ }^{135}$, L.S. Barnby ${ }^{102}$, V. Barret ${ }^{70}$, P. Bartalini ${ }^{7}$, J. Bartke ${ }^{116}$, E. Bartsch ${ }^{53}$, M. Basile ${ }^{28}$, N. Bastid ${ }^{70}$, S. Basu ${ }^{131}$, B. Bathen ${ }^{54}$, G. Batigne ${ }^{113}$, A. Batista Camejo ${ }^{70}$, B. Batyunya ${ }^{66}$, P.C. Batzing ${ }^{22}$, I.G. Bearden ${ }^{80}$, H. Beck ${ }^{53}$, C. Bedda ${ }^{111}$, N.K. Behera ${ }^{48}$, I. Belikov ${ }^{55}$, F. Bellini ${ }^{28}$, H. Bello Martinez ${ }^{2}$, R. Bellwied ${ }^{121}$, R. Belmont ${ }^{134}$, E. Belmont-Moreno ${ }^{64}$, V. Belyaev ${ }^{76}$, G. Bencedi ${ }^{135}$, S. Beole ${ }^{27}$, I. Berceanu ${ }^{78}$, A. Bercuci ${ }^{78}$, Y. Berdnikov ${ }^{85}$, D. Berenyi ${ }^{135}$, R.A. Bertens ${ }^{57}$, D. Berzano ${ }^{36}, 27$, L. Betev ${ }^{36}$, A. Bhasin ${ }^{90}$, I.R. Bhat ${ }^{90}$, A.K. Bhati ${ }^{87}$, B. Bhattacharjee ${ }^{45}$, J. Bhom ${ }^{127}$, L. Bianchi ${ }^{27}, 121$, N. Bianchi ${ }^{72}$, C. Bianchin ${ }^{134}, 57$, J. Bielčík ${ }^{40}$, J. Bielčíková ${ }^{83}$, A. Bilandzic ${ }^{80}$, S. Biswas ${ }^{79}$, S. Bjelogrlic ${ }^{57}$, F. Blanco ${ }^{10}$, D. Blau ${ }^{100}$, C. Blume ${ }^{53}$, F. Bock ${ }^{74}, 93$, A. Bogdanov ${ }^{76}$, H. Bøggild ${ }^{80}$, L. Boldizsár ${ }^{135}$, M. Bombara ${ }^{41}$, J. Book ${ }^{53}$, H. Borel ${ }^{15}$, A. Borissov ${ }^{96}$, M. Borri ${ }^{82}$, F. Bossú ${ }^{65}$, M. Botje ${ }^{81}$, E. Botta ${ }^{27}$, S. Böttger ${ }^{52}$, P. Braun-Munzinger ${ }^{97}$, M. Bregant ${ }^{119}$, T. Breitner ${ }^{52}$, T.A. Broker ${ }^{53}$, T.A. Browning ${ }^{95}$, M. Broz ${ }^{40}$, E.J. Brucken ${ }^{46}$, E. Bruna ${ }^{111}$, G.E. Bruno ${ }^{33}$, D. Budnikov ${ }^{99}$, H. Buesching ${ }^{53}$, S. Bufalino ${ }^{36}, 111$, P. Buncic ${ }^{36}$, O. Busch ${ }^{93}$, Z. Buthelezi ${ }^{65}$, J.T. Buxton ${ }^{20}$, D. Caffarri ${ }^{36}, 30$, X. Cai ${ }^{7}$, H. Caines ${ }^{136}$, L. Calero Diaz ${ }^{72}$, A. Caliva ${ }^{57}$, E. Calvo Villar ${ }^{103}$, P. Camerini ${ }^{26}$, F. Carena ${ }^{36}$, W. Carena ${ }^{36}$, J. Castillo Castellanos ${ }^{15}$, A.J. Castro ${ }^{124}$, E.A.R. Casula ${ }^{25}$, C. Cavicchioli ${ }^{36}$, C. Ceballos Sanchez ${ }^{9}$, J. Cepila ${ }^{40}$, P. Cerello ${ }^{111}$, B. Chang ${ }^{122}$, S. Chapeland ${ }^{36}$, M. Chartier ${ }^{123}$, J.L. Charvet $^{15}$,

S. Chattopadhyay ${ }^{131}$, S. Chattopadhyay ${ }^{101}$, V. Chelnokov ${ }^{3}$, M. Cherney ${ }^{86}$, C. Cheshkov ${ }^{129}$, B. Cheynis ${ }^{129}$, V. Chibante Barroso ${ }^{36}$, D.D. Chinellato ${ }^{120}$, P. Chochula ${ }^{36}$, K. Choi ${ }^{96}$, M. Chojnacki ${ }^{80}$, S. Choudhury ${ }^{131}$, P. Christakoglou ${ }^{81}$, C.H. Christensen ${ }^{80}$, P. Christiansen ${ }^{34}$, T. Chujo ${ }^{127}$, S.U. Chung ${ }^{96}$, C. Cicalo ${ }^{106}$, L. Cifarelli ${ }^{12}, 28$, F. Cindolo ${ }^{105}$, J. Cleymans ${ }^{89}$, F. Colamaria ${ }^{33}$, D. Colella ${ }^{33}$, A. Collu ${ }^{25}$, M. Colocci ${ }^{28}$, G. Conesa Balbastre ${ }^{71}$, Z. Conesa del Valle $^{51}$, M.E. Connors ${ }^{136}$, J.G. Contreras ${ }^{11}, 40$, T.M. Cormier ${ }^{84}$, Y. Corrales Morales ${ }^{27}$, I. Cortés Maldonado ${ }^{2}$, P. Cortese ${ }^{32}$, M.R. Cosentino ${ }^{119}$, F. Costa ${ }^{36}$, P. Crochet $^{70}$, R. Cruz Albino $^{11}$, E. Cuautle ${ }^{63}$, L. Cunqueiro ${ }^{36}$, T. Dahms ${ }^{92}, 37$, A. Dainese ${ }^{108}$, A. Danu ${ }^{62}$, D. $\operatorname{Das}^{101}$, I. $\operatorname{Das}^{51}, 101$, S. $\operatorname{Das}^{4}$, A. Dash ${ }^{120}$, S. Dash ${ }^{48}$, S. De ${ }^{131}, 119$, A. De Caro ${ }^{31}, 12$, G. de Cataldo $^{104}$, J. de Cuveland ${ }^{43}$, A. De Falco ${ }^{25}$, D. De Gruttola ${ }^{12}, 31$, N. De Marco ${ }^{111}$, S. De Pasquale $^{31}$, A. Deloff ${ }^{77}$, E. Dénes ${ }^{135}$, G. D’Erasmo ${ }^{33}$, D. Di Bari ${ }^{33}$, A. Di Mauro ${ }^{36}$, P. Di Nezza $^{72}$, M.A. Diaz Corchero ${ }^{10}$, T. Dietel ${ }^{89}$, P. Dillenseger ${ }^{53}$, R. Divià ${ }^{36}$, Ø. Djuvsland ${ }^{18}$, A. Dobrin ${ }^{57}, 81$, T. Dobrowolski 77 , i, D. Domenicis Gimenez ${ }^{119}$, B. Dönigus ${ }^{53}$, O. Dordic ${ }^{22}$, A.K. Dubey ${ }^{131}$, A. Dubla ${ }^{57}$, L. Ducroux ${ }^{129}$, P. Dupieux ${ }^{70}$, R.J. Ehlers ${ }^{136}$, D. Elia ${ }^{104}$, H. Engel ${ }^{52}$, B. Erazmus ${ }^{113}, 36$, D. Eschweiler ${ }^{43}$, B. Espagnon ${ }^{51}$, M. Estienne ${ }^{113}$, S. Esumi ${ }^{127}$, D. Evans ${ }^{102}$, S. Evdokimov ${ }^{112}$, G. Eyyubova ${ }^{40}$, L. Fabbietti ${ }^{37}, 92$, D. Fabris ${ }^{108}$, J. Faivre ${ }^{71}$, A. Fantoni ${ }^{72}$, M. Fasel ${ }^{74}$, L. Feldkamp ${ }^{54}$, D. Felea ${ }^{62}$, A. Feliciello ${ }^{111}$, G. Feofilov ${ }^{130}$, J. Ferencei ${ }^{83}$, A. Fernández Téllez ${ }^{2}$, E.G. Ferreiro ${ }^{17}$, A. Ferretti ${ }^{27}$, A. Festanti ${ }^{30}$, J. Figiel $^{116}$, M.A.S. Figueredo ${ }^{123}$, S. Filchagin ${ }^{99}$, D. Finogeev ${ }^{56}$, F.M. Fionda ${ }^{104}$, E.M. Fiore ${ }^{33}$, M.G. Fleck ${ }^{93}$, M. Floris ${ }^{36}$, S. Foertsch ${ }^{65}$, P. Foka ${ }^{97}$, S. Fokin ${ }^{100}$, E. Fragiacomo ${ }^{110}$, 


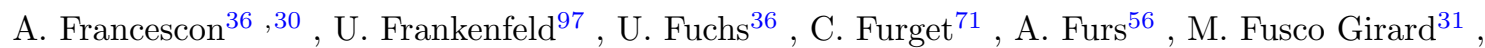
J.J. Gaardhøje ${ }^{80}$, M. Gagliardi ${ }^{27}$, A.M. Gago ${ }^{103}$, M. Gallio ${ }^{27}$, D.R. Gangadharan ${ }^{74}$,

P. Ganoti ${ }^{88}$, C. Gao ${ }^{7}$, C. Garabatos ${ }^{97}$, E. Garcia-Solis ${ }^{13}$, C. Gargiulo ${ }^{36}$, P. Gasik ${ }^{37}, 92$, M. Germain ${ }^{113}$, A. Gheata ${ }^{36}$, M. Gheata ${ }^{62}, 36$, P. Ghosh ${ }^{131}$, S.K. Ghosh ${ }^{4}$, P. Gianotti ${ }^{72}$, P. Giubellino ${ }^{36}$, P. Giubilato ${ }^{30}$, E. Gladysz-Dziadus ${ }^{116}$, P. Glässel ${ }^{93}$, A. Gomez Ramirez ${ }^{52}$, P. González-Zamora ${ }^{10}$, S. Gorbunov ${ }^{43}$, L. Görlich ${ }^{116}$, S. Gotovac ${ }^{115}$, V. Grabski ${ }^{64}$, L.K. Graczykowski ${ }^{133}$, A. Grelli ${ }^{57}$, A. Grigoras ${ }^{36}$, C. Grigoras ${ }^{36}$, V. Grigoriev ${ }^{76}$,

A. Grigoryan ${ }^{1}$, S. Grigoryan ${ }^{66}$, B. Grinyov ${ }^{3}$, N. Grion ${ }^{110}$, J.F. Grosse-Oetringhaus ${ }^{36}$, J.-Y. Grossiord ${ }^{129}$, R. Grosso ${ }^{36}$, F. Guber ${ }^{56}$, R. Guernane ${ }^{71}$, B. Guerzoni ${ }^{28}$, K. Gulbrandsen ${ }^{80}$, H. Gulkanyan ${ }^{1}$, T. Gunji ${ }^{126}$, A. Gupta ${ }^{90}$, R. Gupta ${ }^{90}$, R. Haake ${ }^{54}$, Ø. Haaland ${ }^{18}$,

C. Hadjidakis ${ }^{51}$, M. Haiduc ${ }^{62}$, H. Hamagaki ${ }^{126}$, G. Hamar ${ }^{135}$, L.D. Hanratty ${ }^{102}$, A. Hansen ${ }^{80}$, J.W. Harris ${ }^{136}$, H. Hartmann ${ }^{43}$, A. Harton ${ }^{13}$, D. Hatzifotiadou ${ }^{105}$, S. Hayashi ${ }^{126}$,

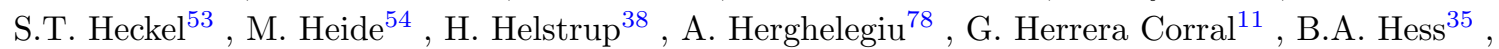
K.F. Hetland ${ }^{38}$, T.E. Hilden ${ }^{46}$, H. Hillemanns ${ }^{36}$, B. Hippolyte ${ }^{55}$, P. Hristov ${ }^{36}$, M. Huang ${ }^{18}$, T.J. Humanic ${ }^{20}$, N. Hussain ${ }^{45}$, T. Hussain ${ }^{19}$, D. Hutter ${ }^{43}$, D.S. Hwang ${ }^{21}$, R. Ilkaev ${ }^{99}$,

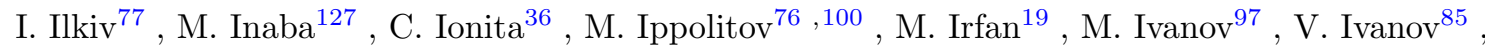
P.M. Jacobs ${ }^{74}$, C. Jahnke ${ }^{119}$, H.J. Jang 68 , M.A. Janik ${ }^{133}$, P.H.S.Y. Jayarathna ${ }^{121}$, C. Jena ${ }^{30}$, S. Jena ${ }^{121}$, R.T. Jimenez Bustamante ${ }^{63}$, P.G. Jones ${ }^{102}$, H. Jung ${ }^{44}$, A. Jusko ${ }^{102}$, P. Kalinak ${ }^{59}$, A. Kalweit ${ }^{36}$, J. Kamin ${ }^{53}$, J.H. Kang ${ }^{137}$, V. Kaplin ${ }^{76}$, S. Kar ${ }^{131}$, A. Karasu Uysal ${ }^{69}$, O. Karavichev ${ }^{56}$, T. Karavicheva ${ }^{56}$, E. Karpechev ${ }^{56}$, U. Kebschull ${ }^{52}$, R. Keidel ${ }^{138}$, D.L.D. Keijdener ${ }^{57}$, M. Keil ${ }^{36}$, K.H. Khan ${ }^{16}$, M.M. Khan ${ }^{19}$, P. Khan ${ }^{101}$, S.A. Khan ${ }^{131}$,

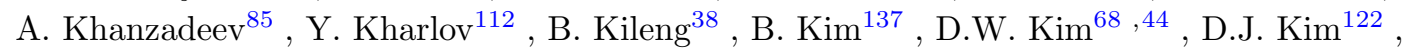

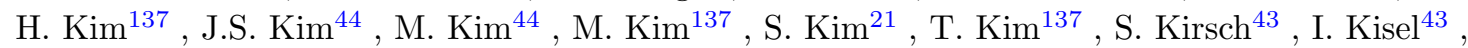
S. Kiselev ${ }^{58}$, A. Kisiel ${ }^{133}$, G. Kiss ${ }^{135}$, J.L. Klay ${ }^{6}$, C. Klein ${ }^{53}$, J. Klein ${ }^{93}$, C. Klein-Bösing ${ }^{54}$, A. Kluge ${ }^{36}$, M.L. Knichel ${ }^{93}$, A.G. Knospe ${ }^{117}$, T. Kobayashi ${ }^{127}$, C. Kobdaj ${ }^{114}$, M. Kofarago ${ }^{36}$, M.K. Köhler ${ }^{97}$, T. Kollegger ${ }^{97}, 43$, A. Kolojvari ${ }^{130}$, V. Kondratiev ${ }^{130}$, N. Kondratyeva ${ }^{76}$, E. Kondratyuk ${ }^{112}$, A. Konevskikh ${ }^{56}$, M. Kour ${ }^{90}$, C. Kouzinopoulos ${ }^{36}$, V. Kovalenko ${ }^{130}$, M. Kowalski ${ }^{36}, 116$, S. Kox ${ }^{71}$, G. Koyithatta Meethaleveedu ${ }^{48}$, J. Kral ${ }^{122}$, I. Králik ${ }^{59}$,

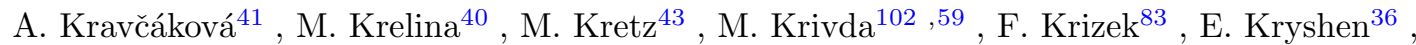
M. Krzewicki ${ }^{97}, 43$, A.M. Kubera ${ }^{20}$, V. Kučera ${ }^{83}$, Y. Kucheriaev ${ }^{100}$, i , T. Kugathasan ${ }^{36}$,

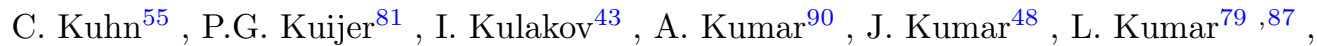
P. Kurashvili 77 , A. Kurepin ${ }^{56}$, A.B. Kurepin ${ }^{56}$, A. Kuryakin ${ }^{99}$, S. Kushpil ${ }^{83}$, M.J. Kweon ${ }^{50}$, Y. Kwon ${ }^{137}$, S.L. La Pointe ${ }^{111}$, P. La Rocca ${ }^{29}$, C. Lagana Fernandes ${ }^{119}$, I. Lakomov ${ }^{51,36}$,

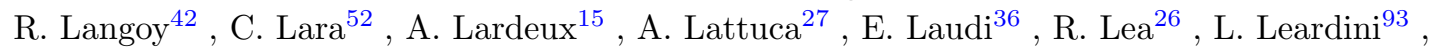
G.R. Lee ${ }^{102}$, S. Lee ${ }^{137}$, I. Legrand ${ }^{36}$, J. Lehnert ${ }^{53}$, R.C. Lemmon ${ }^{82}$, V. Lenti ${ }^{104}$,

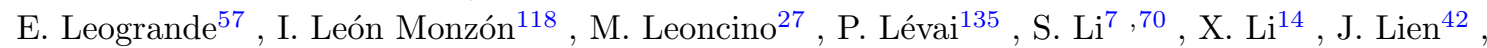
R. Lietava ${ }^{102}$, S. Lindal ${ }^{22}$, V. Lindenstruth ${ }^{43}$, C. Lippmann ${ }^{97}$, M.A. Lisa ${ }^{20}$, H.M. Ljunggren ${ }^{34}$, D.F. Lodato ${ }^{57}$, P.I. Loenne ${ }^{18}$, V.R. Loggins ${ }^{134}$, V. $\operatorname{Loginov}^{76}$, C. Loizides $^{74}$, X. Lopez ${ }^{70}$,

E. López Torres ${ }^{9}$, A. Lowe ${ }^{135}$, X.-G. Lu ${ }^{93}$, P. Luettig ${ }^{53}$, M. Lunardon ${ }^{30}$, G. Luparello ${ }^{26}, 57$, A. Maevskaya ${ }^{56}$, M. Mager ${ }^{36}$, S. Mahajan ${ }^{90}$, S.M. Mahmood ${ }^{22}$, A. Maire ${ }^{55}$, R.D. Majka ${ }^{136}$, M. Malaev ${ }^{85}$, I. Maldonado Cervantes ${ }^{63}$, L. Malinina, ii,66 , D. Mal'Kevich ${ }^{58}$, P. Malzacher ${ }^{97}$, A. Mamonov ${ }^{99}$, L. Manceau ${ }^{111}$, V. Manko ${ }^{100}$,F. Manso ${ }^{70}$, V. Manzari ${ }^{104,36}$, M. Marchisone ${ }^{27}$, J. Mareš ${ }^{60}$, G.V. Margagliotti2 ${ }^{26}$, A. Margotti ${ }^{105}$, J. Margutti ${ }^{57}$, A. Marín ${ }^{97}$, C. Markert ${ }^{117}$, M. Marquard ${ }^{53}$, I. Martashvili ${ }^{124}$, N.A. Martin ${ }^{97}$, J. Martin Blanco ${ }^{113}$, P. Martinengo ${ }^{36}$, M.I. Martínez ${ }^{2}$, G. Martínez García ${ }^{113}$, M. Martinez Pedreira ${ }^{36}$, Y. Martynov ${ }^{3}$, A. Mas ${ }^{119}$, S. Masciocchi ${ }^{97}$, M. Masera ${ }^{27}$, A. Masoni ${ }^{106}$, L. Massacrier ${ }^{113}$, A. Mastroserio ${ }^{33}$, A. Matyja ${ }^{116}$, C. Mayer ${ }^{116}$, J. Mazer ${ }^{124}$, M.A. Mazzoni ${ }^{109}$, D. Mcdonald ${ }^{121}$, F. Meddi ${ }^{24}$, A. Menchaca-Rocha ${ }^{64}$, E. Meninno ${ }^{31}$, J. Mercado Pérez ${ }^{93}$, M. Meres ${ }^{39}$, Y. Miake ${ }^{127}$, M.M. Mieskolainen ${ }^{46}$, K. Mikhaylov ${ }^{58,66}$, L. Milano ${ }^{36}$, J. Milosevic ${ }^{22,132}$, 
L.M. Minervini ${ }^{104}, 23$, A. Mischke ${ }^{57}$, A.N. Mishra ${ }^{49}$, D. Miśkowiec ${ }^{97}$, J. Mitra ${ }^{131}$, C.M. Mitu ${ }^{62}$, N. Mohammadi ${ }^{57}$, B. Mohanty ${ }^{79}, 131$, L. Molnar ${ }^{55}$, L. Montaño Zetina ${ }^{11}$, E. Montes ${ }^{10}$, M. Morando ${ }^{30}$, S. Moretto ${ }^{30}$, A. Morreale ${ }^{113}$, A. Morsch ${ }^{36}$, V. Muccifora ${ }^{72}$, E. Mudnic ${ }^{115}$, D. Mühlheim ${ }^{54}$, S. Muhuri ${ }^{131}$, M. Mukherjee ${ }^{131}$, H. Müller ${ }^{36}$, J.D. Mulligan ${ }^{136}$, M.G. Munhoz ${ }^{119}$, S. Murray ${ }^{65}$, L. Musa ${ }^{36}$, J. Musinsky ${ }^{59}$, B.K. Nandi ${ }^{48}$, R. Nania ${ }^{105}$, E. Nappi ${ }^{104}$, M.U. Naru ${ }^{16}$, C. Nattrass ${ }^{124}$, K. Nayak ${ }^{79}$, T.K. Nayak ${ }^{131}$, S. Nazarenko ${ }^{99}$, A. Nedosekin ${ }^{58}$, L. Nellen ${ }^{63}$, F. Ng ${ }^{121}$, M. Nicassio ${ }^{97}$, M. Niculescu ${ }^{36,62}$, J. Niedziela ${ }^{36}$, B.S. Nielsen ${ }^{80}$, S. Nikolaev ${ }^{100}$, S. Nikulin ${ }^{100}$, V. Nikulin ${ }^{85}$, F. Noferini ${ }^{105}, 12$, P. Nomokonov ${ }^{66}$, G. Nooren ${ }^{57}$, J. Norman ${ }^{123}$, A. Nyanin ${ }^{100}$, J. Nystrand ${ }^{18}$, H. Oeschler ${ }^{93}$, S. Oh ${ }^{136}$, S.K. Oh ${ }^{67}$, A. Ohlson ${ }^{36}$, A. Okatan ${ }^{69}$, T. Okubo ${ }^{47}$, L. Olah ${ }^{135}$, J. Oleniacz ${ }^{133}$, A.C. Oliveira Da Silva ${ }^{119}$, M.H. Oliver ${ }^{136}$, J. Onderwaater ${ }^{97}$, C. Oppedisano ${ }^{111}$, A. Ortiz Velasquez ${ }^{63}$, A. Oskarsson ${ }^{34}$, J. Otwinowski ${ }^{97}, 116$, K. Oyama ${ }^{93}$, M. Ozdemir ${ }^{53}$, Y. Pachmayer ${ }^{93}$, P. Pagano ${ }^{31}$, G. Paić ${ }^{63}$, C. Pajares ${ }^{17}$, S.K. Pal ${ }^{131}$, J. Pan ${ }^{134}$, A.K. Pandey ${ }^{48}$, D. Pant ${ }^{48}$, V. Papikyan ${ }^{1}$, G.S. Pappalardo ${ }^{107}$, P. Pareek ${ }^{49}$, W.J. Park ${ }^{97}$, S. Parmar ${ }^{87}$, A. Passfeld ${ }^{54}$, V. Paticchio ${ }^{104}$, B. Paul ${ }^{101}$, T. Pawlak ${ }^{133}$, T. Peitzmann ${ }^{57}$, H. Pereira Da Costa $^{15}$, E. Pereira De Oliveira Filho $^{119}$, D. Peresunko ${ }^{76}, 100$, C.E. Pérez Lara ${ }^{81}$, V. Peskov ${ }^{53}$, Y. Pestov ${ }^{5}$, V. Petráček ${ }^{40}$, V. Petrov ${ }^{112}$, M. Petrovici ${ }^{78}$, C. Petta ${ }^{29}$, S. Piano ${ }^{110}$, M. Pikna ${ }^{39}$, P. Pillot ${ }^{113}$, O. Pinazza ${ }^{105}, 36$, L. Pinsky ${ }^{121}$, D.B. Piyarathna ${ }^{121}$, M. Płoskoń ${ }^{74}$, M. Planinic ${ }^{128}$, J. Pluta ${ }^{133}$, S. Pochybova ${ }^{135}$, P.L.M. Podesta-Lerma ${ }^{118}$, M.G. Poghosyan ${ }^{86}$, B. Polichtchouk ${ }^{112}$, N. Poljak ${ }^{128}$, W. Poonsawat ${ }^{114}$, A. Pop ${ }^{78}$, S. Porteboeuf-Houssais ${ }^{70}$, J. Porter ${ }^{74}$, J. Pospisil ${ }^{83}$, S.K. Prasad ${ }^{4}$, R. Preghenella ${ }^{105}, 36$, F. Prino ${ }^{111}$, C.A. Pruneau ${ }^{134}$, I. Pshenichnov ${ }^{56}$, M. Puccio ${ }^{111}$, G. Puddu ${ }^{25}$, P. Pujahari ${ }^{134}$, V. Punin ${ }^{99}$, J. Putschke ${ }^{134}$, H. Qvigstad ${ }^{22}$, A. Rachevski ${ }^{110}$, S. Raha ${ }^{4}$, S. Rajput ${ }^{90}$, J. Rak $^{122}$, A. Rakotozafindrabe ${ }^{15}$, L. Ramello ${ }^{32}$, R. Raniwala ${ }^{91}$, S. Raniwala ${ }^{91}$, S.S. Räsänen ${ }^{46}$, B.T. Rascanu ${ }^{53}$, D. Rathee ${ }^{87}$, V. Razazi ${ }^{25}$, K.F. Read ${ }^{124}$, J.S. Real ${ }^{71}$, K. Redlich ${ }^{77}$, R.J. Reed ${ }^{134}$, A. Rehman ${ }^{18}$, P. Reichelt ${ }^{53}$, M. Reicher ${ }^{57}$, F. Reidt ${ }^{93}{ }^{36}$, X. $\operatorname{Ren}^{7}$, R. Renfordt ${ }^{53}$, A.R. Reolon ${ }^{72}$, A. Reshetin ${ }^{56}$, F. Rettig ${ }^{43}$, J.-P. Revol ${ }^{12}$, K. Reygers ${ }^{93}$, V. Riabov ${ }^{85}$, R.A. Ricci ${ }^{73}$, T. Richert ${ }^{34}$, M. Richter ${ }^{22}$, P. Riedler ${ }^{36}$, W. Riegler ${ }^{36}$, F. Riggi ${ }^{29}$, C. Ristea ${ }^{62}$, A. Rivetti ${ }^{111}$, E. Rocco ${ }^{57}$, M. Rodríguez Cahuantzi ${ }^{11}, 2$, A. Rodriguez Manso ${ }^{81}, \mathrm{~K}^{1}$ Røed ${ }^{22}$, E. Rogochaya ${ }^{66}, \mathrm{D}_{\text {. Rohr }}{ }^{43}$, D. Röhrich ${ }^{18}$, R. Romita ${ }^{123}$, F. Ronchetti ${ }^{72}$, L. Ronflette ${ }^{113}$, P. Rosnet ${ }^{70}$, A. Rossi ${ }^{36}$, F. Roukoutakis ${ }^{88}$, A. Roy ${ }^{49}$, C. Roy ${ }^{55}$, P. Roy ${ }^{101}$, A.J. Rubio Montero ${ }^{10}$, R. Rui ${ }^{26}$, R. Russo ${ }^{27}$, E. Ryabinkin ${ }^{100}$, Y. Ryabov ${ }^{85}$, A. Rybicki116 ${ }^{116}$ S. Sadovsky ${ }^{112}$, K. Šafařík ${ }^{36}$, B. Sahlmuller ${ }^{53}$, P. Sahoo ${ }^{49}$, R. Sahoo ${ }^{49}$, S. Sahoo ${ }^{61}$, P.K. Sahu ${ }^{61}$, J. Saini ${ }^{131}$, S. Sakai ${ }^{72}$, M.A. Saleh ${ }^{134}$, C.A. Salgado ${ }^{17}$, J. Salzwedel ${ }^{20}$, S. Sambyal ${ }^{90}$, V. Samsonov ${ }^{85}$, X. Sanchez Castro $^{55}$, L. Šándor ${ }^{59}$, A. Sandoval ${ }^{64}$, M. Sano ${ }^{127}$, G. Santagati ${ }^{29}$, D. Sarkar ${ }^{131}$,

E. Scapparone ${ }^{105}$, F. Scarlassara ${ }^{30}$, R.P. Scharenberg ${ }^{95}$, C. Schiaua ${ }^{78}$, R. Schicker $^{93}$, C. Schmidt ${ }^{97}$, H.R. Schmidt ${ }^{35}$, S. Schuchmann ${ }^{53}$, J. Schukraft ${ }^{36}$, M. Schulc ${ }^{40}$, T. Schuster ${ }^{136}$,

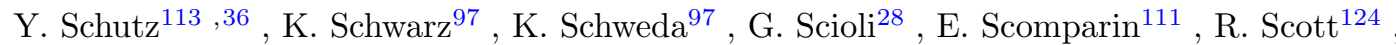
K.S. Seeder ${ }^{119}$, J.E. Seger ${ }^{86}$, Y. Sekiguchi ${ }^{126}$, I. Selyuzhenkov ${ }^{97}$, K. Senosi ${ }^{65}$, J. Seo ${ }^{67}, 96$, E. Serradilla ${ }^{10}, 64$, A. Sevcenco ${ }^{62}$, A. Shabanov ${ }^{56}$, A. Shabetai ${ }^{113}$, O. Shadura ${ }^{3}$, R. Shahoyan ${ }^{36}$, A. Shangaraev ${ }^{112}$, A. Sharma ${ }^{90}$, M. Sharma ${ }^{90}$, N. Sharma ${ }^{61,124}$, K. Shigaki ${ }^{47}$, K. Shtejer ${ }^{27,9}$, Y. Sibiriak ${ }^{100}$, S. Siddhanta ${ }^{106}$, K.M. Sielewicz ${ }^{36}$, T. Siemiarczuk ${ }^{77}$, D. Silvermyr ${ }^{84}, 34$, C. Silvestre ${ }^{71}$, G. Simatovic ${ }^{128}$, G. Simonetti ${ }^{36}$, R. Singaraju ${ }^{131}$, R. Singh ${ }^{90}, 79$, S. Singha ${ }^{79}, 131$, V. Singhal ${ }^{131}$, B.C. Sinha ${ }^{131}$, T. Sinha ${ }^{101}$, B. Sitar ${ }^{39}$, M. Sitta ${ }^{32}$, T.B. Skaali ${ }^{22}$, M. Slupecki ${ }^{122}$, N. Smirnov ${ }^{136}$, R.J.M. Snellings ${ }^{57}$, T.W. Snellman ${ }^{122}$, C. Søgaard ${ }^{34}$, R. Soltz ${ }^{75}$, J. Song ${ }^{96}$, M. Song ${ }^{137}$, Z. Song ${ }^{7}$, F. Soramel ${ }^{30}$, S. Sorensen ${ }^{124}$, M. Spacek ${ }^{40}$, E. Spiriti ${ }^{72}$, I. Sputowska ${ }^{116}$, M. Spyropoulou-Stassinaki ${ }^{88}$, B.K. Srivastava ${ }^{95}$, J. Stachel ${ }^{93}$, I. Stan ${ }^{62}$, G. Stefanek ${ }^{77}$, M. Steinpreis ${ }^{20}$, E. Stenlund ${ }^{34}$, G. Steyn ${ }^{65}$, J.H. Stiller ${ }^{93}$, D. Stocco ${ }^{113}$, P. Strmen ${ }^{39}$, A.A.P. Suaide ${ }^{119}$, T. Sugitate ${ }^{47}$, 
C. Suire ${ }^{51}$, M. Suleymanov ${ }^{16}$, R. Sultanov ${ }^{58}$, M. Šumbera ${ }^{83}$, T.J.M. Symons ${ }^{74}$, A. Szabo ${ }^{39}$,

A. Szanto de Toledo ${ }^{119}$, i , I. Szarka ${ }^{39}$, A. Szczepankiewicz ${ }^{36}$, M. Szymanski ${ }^{133}$, J. Takahashi ${ }^{120}$, N. Tanaka ${ }^{127}$, M.A. Tangaro ${ }^{33}$, J.D. Tapia Takaki, iii,51, A. Tarantola Peloni ${ }^{53}$, M. Tariq ${ }^{19}$, M.G. Tarzila ${ }^{78}$, A. Tauro ${ }^{36}$, G. Tejeda Muñoz ${ }^{2}$, A. Telesca ${ }^{36}$, K. Terasaki ${ }^{126}$, C. Terrevoli ${ }^{30}, 25$, B. Teyssier ${ }^{129}$, J. Thäder ${ }^{97}, 74$, D. Thomas ${ }^{57,117}$, R. Tieulent ${ }^{129}$, A.R. Timmins ${ }^{121}$, A. Toia ${ }^{53}$, S. Trogolo ${ }^{111}$, V. Trubnikov ${ }^{3}$, W.H. Trzaska ${ }^{122}$, T. Tsuji ${ }^{126}$, A. Tumkin ${ }^{99}$, R. Turrisi ${ }^{108}$, T.S. Tveter ${ }^{22}$, K. Ullaland ${ }^{18}$, A. Uras ${ }^{129}$, G.L. Usai ${ }^{25}$, A. Utrobicic ${ }^{128}$, M. Vajzer ${ }^{83}$, M. Vala ${ }^{59}$, L. Valencia Palomo ${ }^{70}$, S. Vallero ${ }^{27}$, J. Van Der

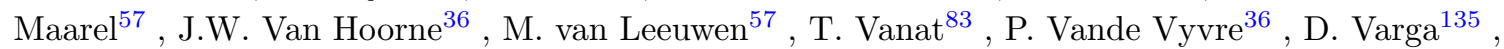

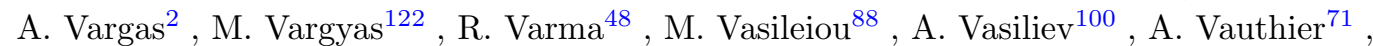
V. Vechernin ${ }^{130}$, A.M. Veen ${ }^{57}$, M. Veldhoen ${ }^{57}$, A. Velure ${ }^{18}$, M. Venaruzzo ${ }^{73}$, E. Vercellin ${ }^{27}$, S. Vergara Limón ${ }^{2}$, R. Vernet ${ }^{8}$, M. Verweij ${ }^{134}$, L. Vickovic ${ }^{115}$, G. Viesti ${ }^{30}$, i, J. Viinikainen ${ }^{122}$, Z. Vilakazi ${ }^{125}$, O. Villalobos Baillie ${ }^{102}$, A. Vinogradov ${ }^{100}$, L. Vinogradov ${ }^{130}$, Y. Vinogradov ${ }^{99}$, i, T. Virgili ${ }^{31}$, V. Vislavicius ${ }^{34}$, Y.P. Viyogi ${ }^{131}$, A. Vodopyanov ${ }^{66}$, M.A. Völkl ${ }^{93}$, K. Voloshin ${ }^{58}$, S.A. Voloshin ${ }^{134}$, G. Volpe ${ }^{135}, 36$, B. von Haller ${ }^{36}$, I. Vorobyev ${ }^{92}, 37$, D. Vranic ${ }^{97}, 36$, J. Vrlákováa ${ }^{41}$, B. Vulpescu ${ }^{70}$, A. Vyushin ${ }^{99}$, B. Wagner ${ }^{18}$, J. Wagner ${ }^{97}$, H. Wang ${ }^{57}$, M. Wang ${ }^{7}, 113$, Y. Wang ${ }^{93}$, D. Watanabe ${ }^{127}$, M. Weber ${ }^{36,121}$, S.G. Weber ${ }^{97}$, J.P. Wessels ${ }^{54}$, U. Westerhoff ${ }^{54}$, J. Wiechula ${ }^{35}$, J. Wikne ${ }^{22}$, M. Wilde ${ }^{54}$, G. Wilk ${ }^{77}$, J. Wilkinson ${ }^{93}$, M.C.S. Williams ${ }^{105}$, B. Windelband ${ }^{93}$, M. Winn ${ }^{93}$, C.G. Yaldo ${ }^{134}$, Y. Yamaguchi ${ }^{126}$, H. Yang ${ }^{57}$, P. Yang ${ }^{7}$, S. Yano ${ }^{47}$, S. Yasnopolskiy ${ }^{100}$, Z. Yin ${ }^{7}$,

H. Yokoyama ${ }^{127}$, I.-K. Yoo ${ }^{96}$, V. Yurchenko ${ }^{3}$, I. Yushmanov ${ }^{100}$, A. Zaborowska ${ }^{133}$, V. Zaccolo ${ }^{80}$, A. Zaman ${ }^{16}$, C. Zampolli ${ }^{105}$, H.J.C. Zanoli ${ }^{119}$, S. Zaporozhets ${ }^{66}$,

A. Zarochentsev ${ }^{130}$, P. Závada ${ }^{60}$, N. Zaviyalov ${ }^{99}$, H. Zbroszczyk ${ }^{133}$, I.S. Zgura ${ }^{62}$, M. Zhalov ${ }^{85}$, H. Zhang ${ }^{18}, 7$, X. Zhang ${ }^{74}$, Y. Zhang ${ }^{7}$, C. Zhao ${ }^{22}$, N. Zhigareva ${ }^{58}$, D. Zhou ${ }^{7}$, Y. Zhou ${ }^{57}$, Z. Zhou ${ }^{18}$, H. Zhu ${ }^{7}, 18$, J. Zhu ${ }^{7}{ }^{113}$, X. Zhu ${ }^{7}$, A. Zichichi ${ }^{12,28}$, A. Zimmermann ${ }^{93}$, M.B. Zimmermann ${ }^{54}, 36$, G. Zinovjev ${ }^{3}$, M. Zyzak ${ }^{43}$

i Deceased

ii Also at: M.V. Lomonosov Moscow State University, D.V. Skobeltsyn Institute of Nuclear, Physics, Moscow, Russia

iii Also at: University of Kansas, Lawrence, Kansas, United States

1 A.I. Alikhanyan National Science Laboratory (Yerevan Physics Institute) Foundation, Yerevan, Armenia

2 Benemérita Universidad Autónoma de Puebla, Puebla, Mexico

3 Bogolyubov Institute for Theoretical Physics, Kiev, Ukraine

4 Bose Institute, Department of Physics and Centre for Astroparticle Physics and Space Science (CAPSS), Kolkata, India

5 Budker Institute for Nuclear Physics, Novosibirsk, Russia

6 California Polytechnic State University, San Luis Obispo, California, United States

Central China Normal University, Wuhan, China

Centre de Calcul de l'IN2P3, Villeurbanne, France

Centro de Aplicaciones Tecnológicas y Desarrollo Nuclear (CEADEN), Havana, Cuba

Centro de Investigaciones Energéticas Medioambientales y Tecnológicas (CIEMAT), Madrid, Spain

Centro de Investigación y de Estudios Avanzados (CINVESTAV), Mexico City and Mérida, Mexico

Centro Fermi - Museo Storico della Fisica e Centro Studi e Ricerche "Enrico Fermi", Rome, Italy

Chicago State University, Chicago, Illinois, U.S.A.

China Institute of Atomic Energy, Beijing, China

Commissariat à l'Energie Atomique, IRFU, Saclay, France 
COMSATS Institute of Information Technology (CIIT), Islamabad, Pakistan

Departamento de Física de Partículas and IGFAE, Universidad de Santiago de Compostela, Santiago de Compostela, Spain

Department of Physics and Technology, University of Bergen, Bergen, Norway

Department of Physics, Aligarh Muslim University, Aligarh, India

Department of Physics, Ohio State University, Columbus, Ohio, United States

Department of Physics, Sejong University, Seoul, South Korea

Department of Physics, University of Oslo, Oslo, Norway

Dipartimento di Elettrotecnica ed Elettronica del Politecnico, Bari, Italy

Dipartimento di Fisica dell'Università 'La Sapienza' and Sezione INFN Rome, Italy

Dipartimento di Fisica dell'Università and Sezione INFN, Cagliari, Italy

Dipartimento di Fisica dell'Università and Sezione INFN, Trieste, Italy

Dipartimento di Fisica dell'Università and Sezione INFN, Turin, Italy

Dipartimento di Fisica e Astronomia dell'Università and Sezione INFN, Bologna, Italy

Dipartimento di Fisica e Astronomia dell'Università and Sezione INFN, Catania, Italy

Dipartimento di Fisica e Astronomia dell'Università and Sezione INFN, Padova, Italy

Dipartimento di Fisica 'E.R. Caianiello' dell'Università and Gruppo Collegato INFN, Salerno, Italy

Dipartimento di Scienze e Innovazione Tecnologica dell'Università del Piemonte Orientale and

Gruppo Collegato INFN, Alessandria, Italy

Dipartimento Interateneo di Fisica 'M. Merlin' and Sezione INFN, Bari, Italy

Division of Experimental High Energy Physics, University of Lund, Lund, Sweden

Eberhard Karls Universität Tübingen, Tübingen, Germany

European Organization for Nuclear Research (CERN), Geneva, Switzerland

Excellence Cluster Universe, Technische Universität München, Munich, Germany

Faculty of Engineering, Bergen University College, Bergen, Norway

Faculty of Mathematics, Physics and Informatics, Comenius University, Bratislava, Slovakia

Faculty of Nuclear Sciences and Physical Engineering, Czech Technical University in Prague,

Prague, Czech Republic

Faculty of Science, P.J. Šafárik University, Košice, Slovakia

Faculty of Technology, Buskerud and Vestfold University College, Vestfold, Norway

Frankfurt Institute for Advanced Studies, Johann Wolfgang Goethe-Universität Frankfurt,

Frankfurt, Germany

Gangneung-Wonju National University, Gangneung, South Korea

Gauhati University, Department of Physics, Guwahati, India

Helsinki Institute of Physics (HIP), Helsinki, Finland

Hiroshima University, Hiroshima, Japan

Indian Institute of Technology Bombay (IIT), Mumbai, India

Indian Institute of Technology Indore, Indore (IITI), India

Inha University, Incheon, South Korea

Institut de Physique Nucléaire d'Orsay (IPNO), Université Paris-Sud, CNRS-IN2P3, Orsay, France Institut für Informatik, Johann Wolfgang Goethe-Universität Frankfurt, Frankfurt, Germany

Institut für Kernphysik, Johann Wolfgang Goethe-Universität Frankfurt, Frankfurt, Germany

Institut für Kernphysik, Westfälische Wilhelms-Universität Münster, Münster, Germany

Institut Pluridisciplinaire Hubert Curien (IPHC), Université de Strasbourg, CNRS-IN2P3,

Strasbourg, France

Institute for Nuclear Research, Academy of Sciences, Moscow, Russia

Institute for Subatomic Physics of Utrecht University, Utrecht, Netherlands

Institute for Theoretical and Experimental Physics, Moscow, Russia

Institute of Experimental Physics, Slovak Academy of Sciences, Kořice, Slovakia

Institute of Physics, Academy of Sciences of the Czech Republic, Prague, Czech Republic

Institute of Physics, Bhubaneswar, India

Institute of Space Science (ISS), Bucharest, Romania

Instituto de Ciencias Nucleares, Universidad Nacional Autónoma de México, Mexico City, Mexico 
Instituto de Física, Universidad Nacional Autónoma de México, Mexico City, Mexico iThemba LABS, National Research Foundation, Somerset West, South Africa

Joint Institute for Nuclear Research (JINR), Dubna, Russia

Konkuk University, Seoul, South Korea

Korea Institute of Science and Technology Information, Daejeon, South Korea

KTO Karatay University, Konya, Turkey

Laboratoire de Physique Corpusculaire (LPC), Clermont Université, Université Blaise Pascal, CNRS-IN2P3, Clermont-Ferrand, France

71 Laboratoire de Physique Subatomique et de Cosmologie, Université Grenoble-Alpes, CNRS-IN2P3, Grenoble, France

72 Laboratori Nazionali di Frascati, INFN, Frascati, Italy

3 Laboratori Nazionali di Legnaro, INFN, Legnaro, Italy

74 Lawrence Berkeley National Laboratory, Berkeley, California, United States

75 Lawrence Livermore National Laboratory, Livermore, California, United States

76 Moscow Engineering Physics Institute, Moscow, Russia

National Centre for Nuclear Studies, Warsaw, Poland

National Institute for Physics and Nuclear Engineering, Bucharest, Romania

National Institute of Science Education and Research, Bhubaneswar, India

Niels Bohr Institute, University of Copenhagen, Copenhagen, Denmark

1 Nikhef, Nationaal instituut voor subatomaire fysica, Amsterdam, Netherlands

Nuclear Physics Group, STFC Daresbury Laboratory, Daresbury, United Kingdom

83 Nuclear Physics Institute, Academy of Sciences of the Czech Republic, Řež u Prahy, Czech Republic

84 Oak Ridge National Laboratory, Oak Ridge, Tennessee, United States

Petersburg Nuclear Physics Institute, Gatchina, Russia

Physics Department, Creighton University, Omaha, Nebraska, United States

Physics Department, Panjab University, Chandigarh, India

Physics Department, University of Athens, Athens, Greece

Physics Department, University of Cape Town, Cape Town, South Africa

Physics Department, University of Jammu, Jammu, India

Physics Department, University of Rajasthan, Jaipur, India

Physik Department, Technische Universität München, Munich, Germany

Physikalisches Institut, Ruprecht-Karls-Universität Heidelberg, Heidelberg, Germany

Politecnico di Torino, Turin, Italy

Purdue University, West Lafayette, Indiana, United States

Pusan National University, Pusan, South Korea

7 Research Division and ExtreMe Matter Institute EMMI, GSI Helmholtzzentrum für Schwerionenforschung, Darmstadt, Germany

Rudjer Bošković Institute, Zagreb, Croatia

Russian Federal Nuclear Center (VNIIEF), Sarov, Russia

Russian Research Centre Kurchatov Institute, Moscow, Russia

Saha Institute of Nuclear Physics, Kolkata, India

School of Physics and Astronomy, University of Birmingham, Birmingham, United Kingdom

Sección Física, Departamento de Ciencias, Pontificia Universidad Católica del Perú, Lima, Peru

Sezione INFN, Bari, Italy

Sezione INFN, Bologna, Italy

Sezione INFN, Cagliari, Italy

Sezione INFN, Catania, Italy

Sezione INFN, Padova, Italy

Sezione INFN, Rome, Italy

Sezione INFN, Trieste, Italy

Sezione INFN, Turin, Italy

SSC IHEP of NRC Kurchatov institute, Protvino, Russia

SUBATECH, Ecole des Mines de Nantes, Université de Nantes, CNRS-IN2P3, Nantes, France 
Suranaree University of Technology, Nakhon Ratchasima, Thailand

Technical University of Split FESB, Split, Croatia

The Henryk Niewodniczanski Institute of Nuclear Physics, Polish Academy of Sciences, Cracow, Poland

The University of Texas at Austin, Physics Department, Austin, Texas, U.S.A.

Universidad Autónoma de Sinaloa, Culiacán, Mexico

Universidade de São Paulo (USP), São Paulo, Brazil

Universidade Estadual de Campinas (UNICAMP), Campinas, Brazil

University of Houston, Houston, Texas, United States

University of Jyväskylä, Jyväskylä, Finland

University of Liverpool, Liverpool, United Kingdom

University of Tennessee, Knoxville, Tennessee, United States

University of the Witwatersrand, Johannesburg, South Africa

University of Tokyo, Tokyo, Japan

University of Tsukuba, Tsukuba, Japan

University of Zagreb, Zagreb, Croatia

Université de Lyon, Université Lyon 1, CNRS/IN2P3, IPN-Lyon, Villeurbanne, France

V. Fock Institute for Physics, St. Petersburg State University, St. Petersburg, Russia

Variable Energy Cyclotron Centre, Kolkata, India

Vinča Institute of Nuclear Sciences, Belgrade, Serbia

Warsaw University of Technology, Warsaw, Poland

Wayne State University, Detroit, Michigan, United States

Wigner Research Centre for Physics, Hungarian Academy of Sciences, Budapest, Hungary

Yale University, New Haven, Connecticut, United States

Yonsei University, Seoul, South Korea

Zentrum für Technologietransfer und Telekommunikation (ZTT), Fachhochschule Worms, Worms, Germany 